Portland State University

PDXScholar

$11-30-2021$

\title{
Exploring the Potential Use of Porous Silica Nanoparticles in CEST-MRI
}

Lydia Makotamo

Portland State University

Follow this and additional works at: https://pdxscholar.library.pdx.edu/open_access_etds

Part of the Inorganic Chemistry Commons

Let us know how access to this document benefits you.

\section{Recommended Citation}

Makotamo, Lydia, "Exploring the Potential Use of Porous Silica Nanoparticles in CEST-MRI" (2021). Dissertations and Theses. Paper 5858.

https://doi.org/10.15760/etd.7729

This Dissertation is brought to you for free and open access. It has been accepted for inclusion in Dissertations and Theses by an authorized administrator of PDXScholar. Please contact us if we can make this document more accessible: pdxscholar@pdx.edu. 
Exploring the Potential Use of Porous Silica Nanoparticles in CEST-MRI

by

Lydia Makotamo

A dissertation submitted in partial fulfillment of the requirements for the degree of

Doctor of Philosophy

in

Chemistry

Dissertation Committee:

Andrea Goforth, Chair

Mark Woods

Theresa McCormick

Rolf Koenenkamp

Portland State University

2021 


\begin{abstract}
Magnetic resonance imaging (MRI) is a widely used modality in diagnostics, treatment, and monitoring various conditions due to its ability to generate high-resolution images using non-ionizing radiation. The advent of contrast agents, which generally work by increasing the spin relaxation rate constants of water protons, has led to image enhancement. Despite the usefulness of this class of agents in diagnostic medicine, their inability to provide in-depth functional and anatomical information, coupled with their high detection limits, has led to new agents being explored.
\end{abstract}

Chemical exchange saturation transfer (CEST) presents an alternative route to generate signals via repetitive saturations transfers between small molecules and bulk water, thus amplifying the signal and making it possible to detect small concentrations. Encapsulating a paramagnetic chemical shift agent in a nanoparticle with a large amount of water allows for simultaneous selective saturation of a large number of water protons. When these protons are allowed to exchange with bulk water protons in a controlled manner, the sensitivity improves, lowering the detection limit. Still, the small number of exchanging protons on these CEST agents means the problem of high detection limits persists.

In this thesis project, the goals were to establish synthetic access to two types of mesoporous silica nanoparticles with different internal morphologies and determine whether a solid silica coating can effectively encapsulate charged paramagnetic contrast agents within the interiors of the mesoporous silica nanoparticles. 
Mesoporous silica nanoparticles (MSNs) were developed following a modified literature procedure. Details of how the incorporation of two swelling agents (including one not reported previously) is also presented. The viability of synthesizing the MSNs with and without the swelling agents was also assessed, and reasons for observed variations were discussed. Hollow mesoporous silica nanoparticles (hMSN) were also synthesized using a modified literature-based method which generally includes three steps: solid core synthesis, mesoporous shell deposition, and etching. A delayed-addition Stöber-based procedure for synthesizing the templating silica is also presented, improving size consistency from batch to batch.

With evidence of solid silica being porous to water, both types of nanoparticles were solid silica-coated to effectively trap the load inside. An easily detectable surrogate dye with the same charge as the intended load was used for the studies. Several techniques were used to quantify surface silanol, assess amine functionalization, and evaluate the effectiveness of the coating procedure. 


\section{Dedication}

This thesis is dedicated to my Mom, Tamary, who has been my pillar of strength, support and has sacrificed a lot throughout my education journey despite not getting an education herself. I love you, Mom;

To my uncle and aunt, Itayi and Linience, who saw something in me, believed in me, and provided that first bridge that has gotten me this far. I am super grateful;

Furthermore, my late grandmother Elizabeth, my all-time cheerleader for as long as I remember, always ululating and dancing for me on prize-giving days. I miss you every day. 
Acknowledgments

First and foremost, I would like to praise and thank the Lord, Almighty, for the countless blessings, opportunities, and all the people with whom He has surrounded me throughout this journey. Ebenezer!

I want to express my sincere gratitude to my Supervisor, Dr. Andrea Goforth, who provided me with the time, valuable advice, and countenance to complete this thesis. Thank you for not only being my academic supervisor but providing professional and personal growth support.

Thank you to my committee members, Dr. Theresa McCormick, Dr. Mark Woods, and Rolf Koenenkamp, for your time, stimulating feedback, and valuable insights you provided along the way.

Many thanks go to all my fellow labmates for giving me helpful suggestions from the time I started this journey to the point of completion. I mostly appreciate Dr. Hayden Winter's in-depth discussions, encouragement, and taking most of the beautiful TEM images in this thesis.

This thesis would not have been a success without the help of the following individuals: Emmanuel Abdul, who helped with thermogravimetric studies and assisted in the analysis of nitrogen porosimetry data; Kevin Fabrizio from the University of Oregon, who tirelessly ran the N2 porosimetry samples at a time he was finishing his work. Anne 
Wachana, Sophia Kim, and Dimitri Buckallew, amazing undergrad students I have had the privilege to work with.

Lastly, I would like to acknowledge all the people who surrounded me, my friends, the stockroom staff. Those "how is it going?", "How are you holding up" meant a lot. You made this place "my home away from home" A special shout out to my dear friend Dr. Ian Munhenzva; you are a shining star. Barbara Giesy, thank you for sharing your family with me away from my home country, and to Mindy Johnson, thank you for picking me up when I was down. 
Table of Contents

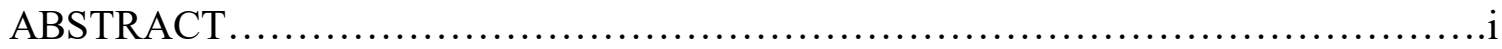

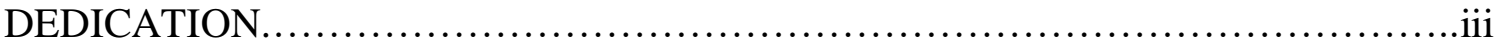

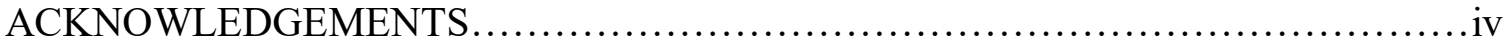

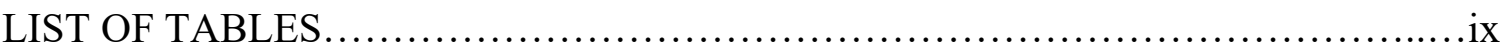

LIST OF FIGURES......................................................

LIST OF ABBREVIATIONS ..................................................

CHAPTER 1: INTRODUCTION TO CHEMICAL EXCHANGE SATURATION TRANSFER, ROLES OF NANOPARTICLES AND SILICA NANOPARTICLES.........1

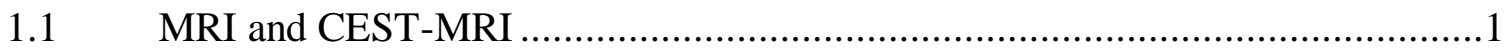

1.2 Nanoparticles in CEST MR imaging ......................................................

$1.3 \quad$ Mesoporous silica as suitable CEST-MRI agent host ..................................11

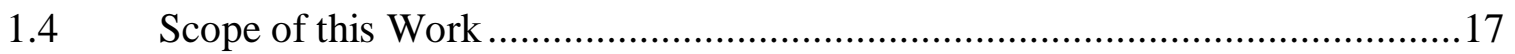

CHAPTER 2: SYNTHESIS OF MESOPOROUS SILICA NANOPARTICLES (MSN) 22

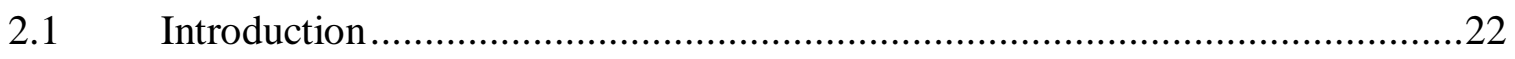

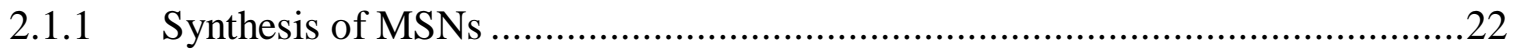

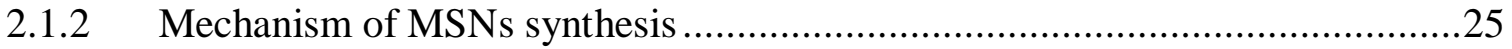

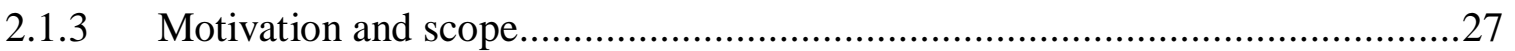

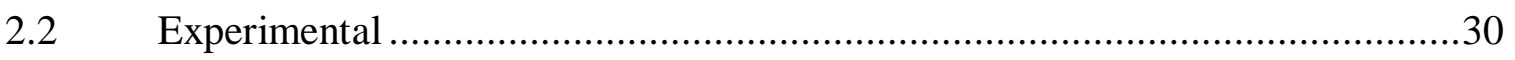

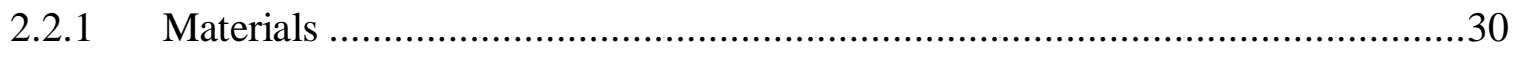

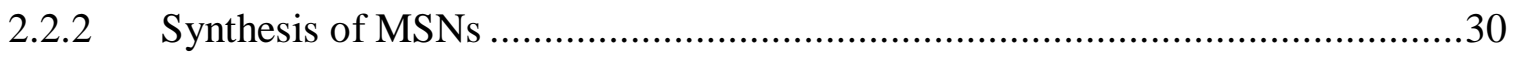

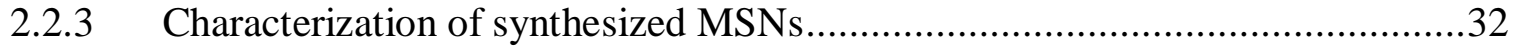

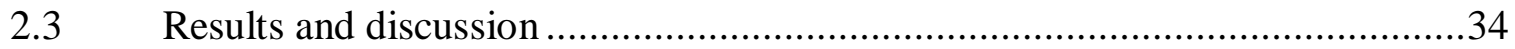

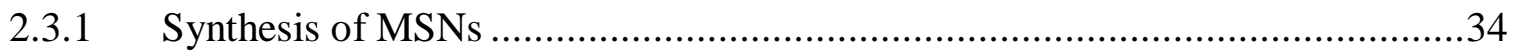

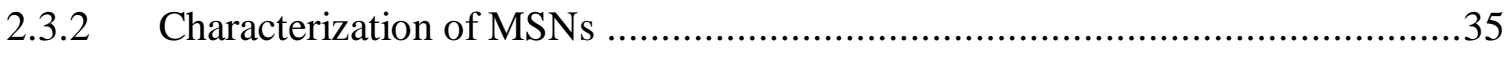

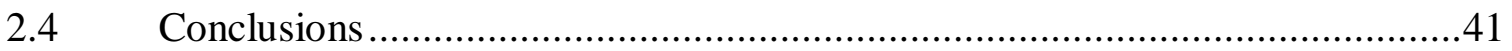

CHAPTER 3: SYNTHESIS OF HOLLOW MESOPOROUS SILICA NANOPARTICLES

.

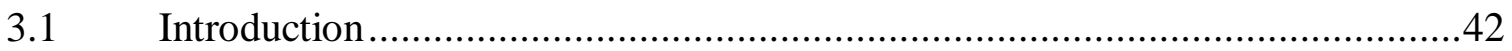




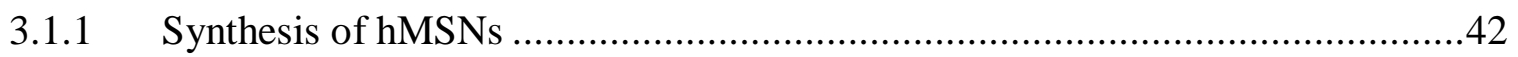

3.1.2 Mechanisms of templated-hMSNs synthesis ................................................

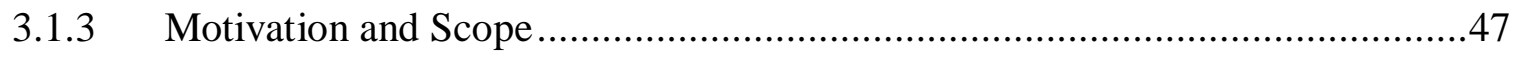

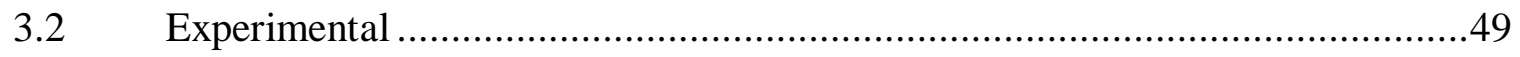

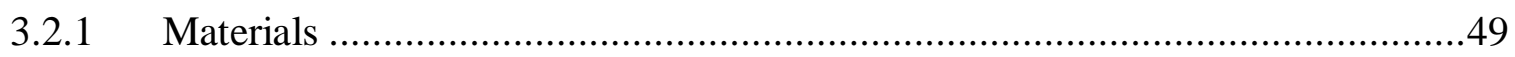

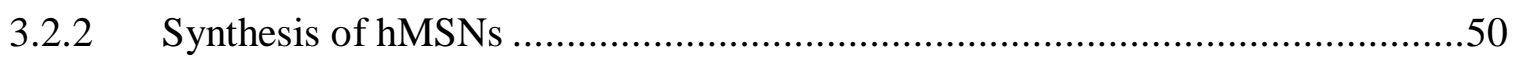

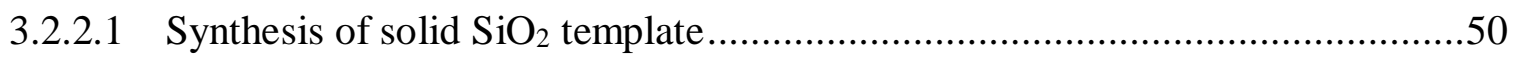

3.2.2.2 Deposition of a mesoporous silica shell .....................................................

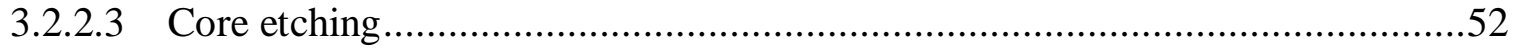

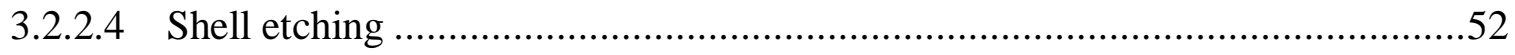

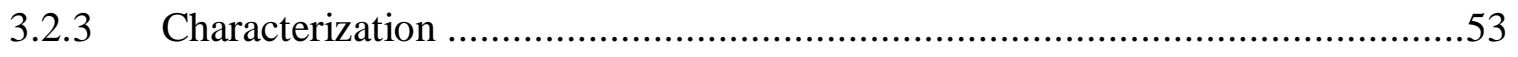

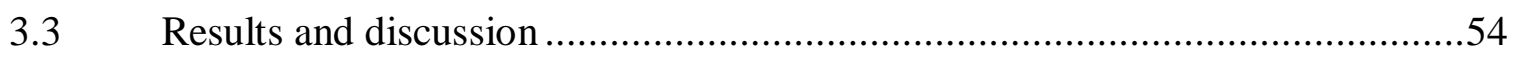

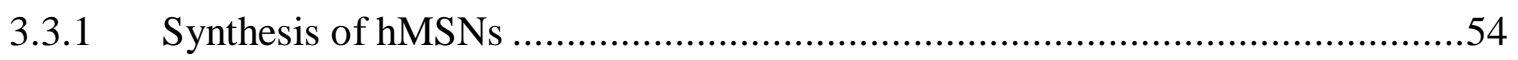

3.3.1.1 Synthesis of Stöber template ……………………......................................56

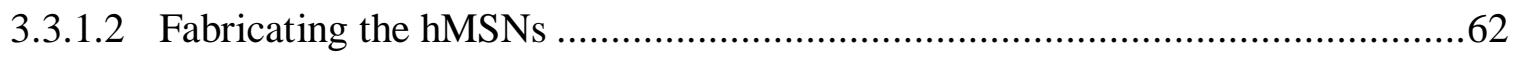

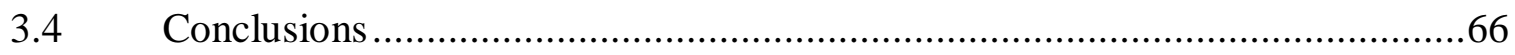

CHAPTER 4: LOADING, COATING, AND QUANTIFICATION OF SURROGATE

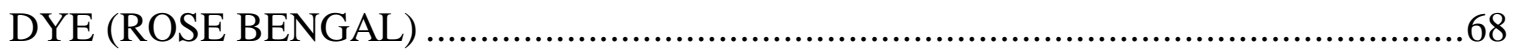

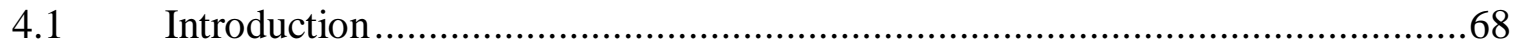

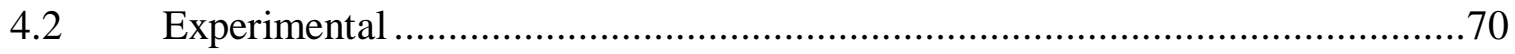

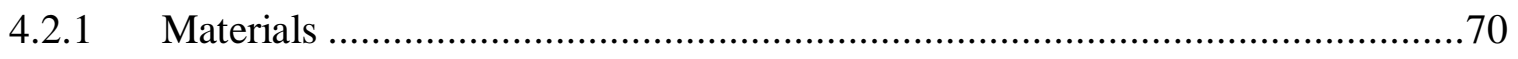

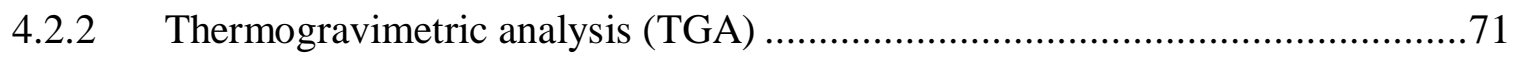

4.2.3 Amine-functionalization of MSNs ............................................................

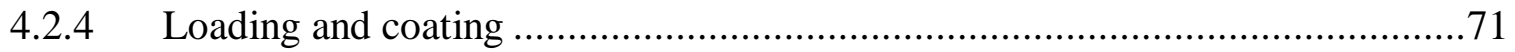

4.2.5 Characterization of loaded and coated MSNs...............................................72

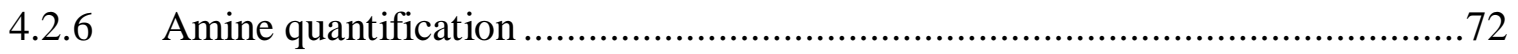

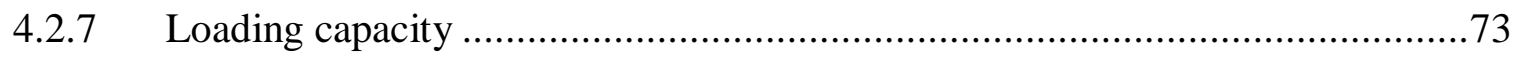

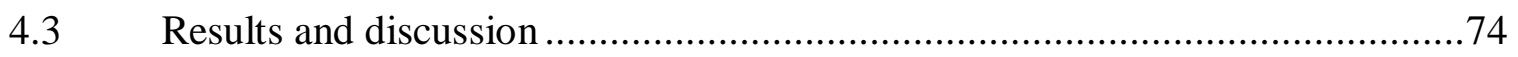

4.3.1 Quantification of surface hydroxyls using TGA ……....................................74

4.3.2 Amine modification of MSNs ....................................................................77 


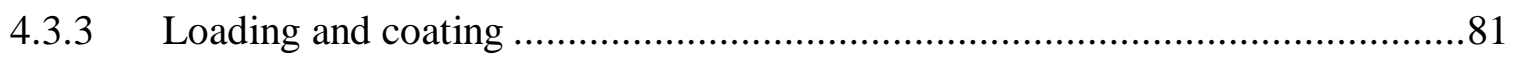

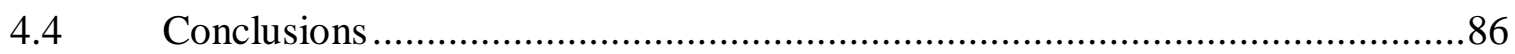

CHAPTER 5: A MODIFIED STÖBER METHOD USING COMMERCIALLY AVAILABLE SILICA SEEDS FOR PRODUCING LARGE, SILICA NANOPARTICLES WITH LOW POLYDISPERSITY AND THEIR SUBSEQUENT USE IN THE SYNTHESIS OF RATTLE-TYPE HOLLOW MESOPOROUS SILICA

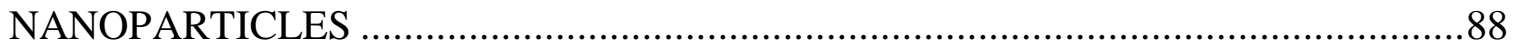

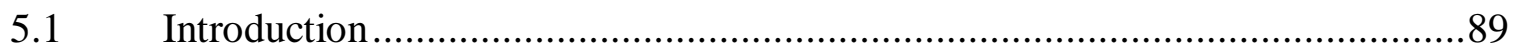

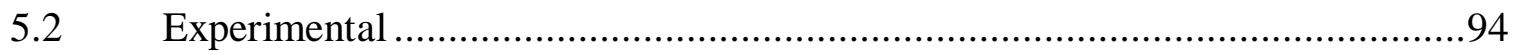

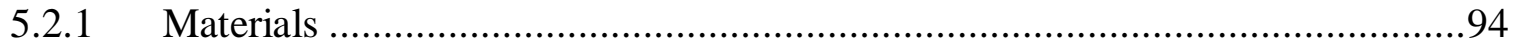

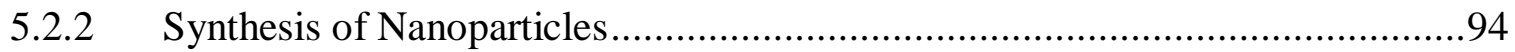

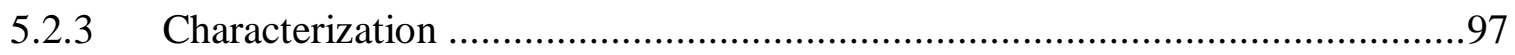

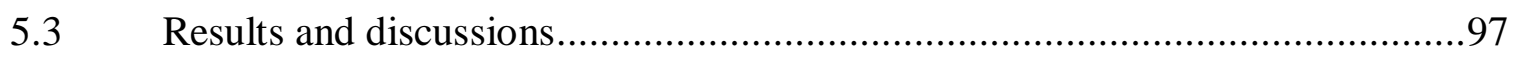

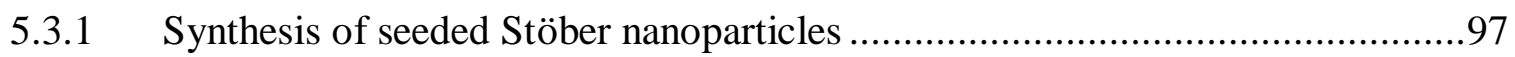

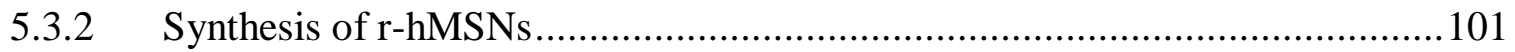

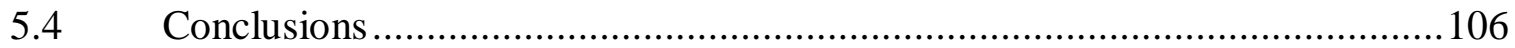

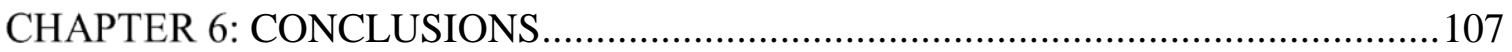

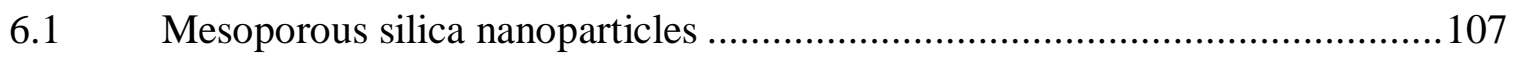

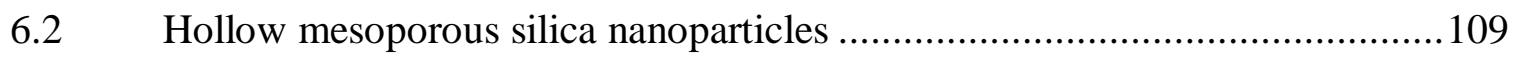

6.3 Surface functionalization, loading, and coating of MSNs ...............................111

6.4 A modified Stöber method using commercially available silica seeds for producing large silica nanoparticles with low size dispersity and their subsequent use in the synthesis of rattle-type hollow mesoporous silica nanoparticles..............................112

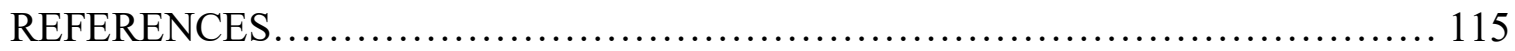


List of Tables

Table 1.1 Examples of nano-based systems that have been explored in CEST-MRI, advantages, and disadvantages.....

Table 2.1. Summary of MSNs morphological characteristics based on starting materials,

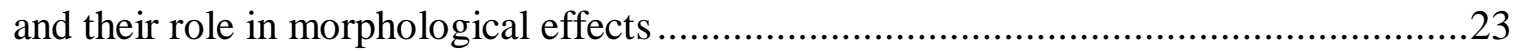

Table 3.1 Starting material concentration for different sized nanoparticles .....................58

Table 4.1 Silanol number calculations for std-MSNs and hMSNs ...............................77

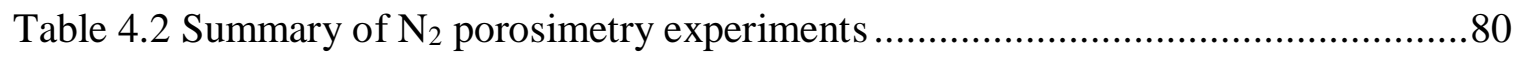

Table 4.3 Summary of loading and coating experiments. ........................................... 83

Table 4.4 Elemental mapping of loaded MSNs showing (atomic \%) ...........................86

Table 5.1 Experimental conditions and results...................................................98 
List of Figures

Figure 1.1 Chemical exchange saturation transfer: principles and measurements for pure

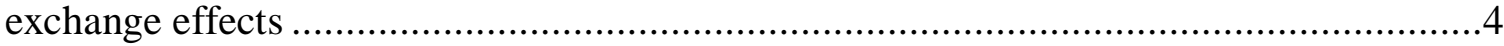

Figure 1.2 Mechanism for a nano-based host in CEST MR imaging ............................

Figure 1.3 The sol-gel method for making $\mathrm{SiO}_{2} \mathrm{NPs}$, here from tetraethoxysilane in

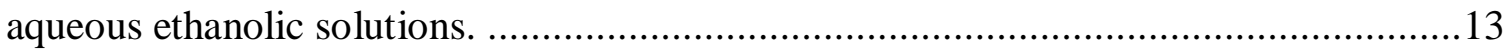

Figure 1.4 Synthetic routes based on the Stöber method for making different SiO2 NPs

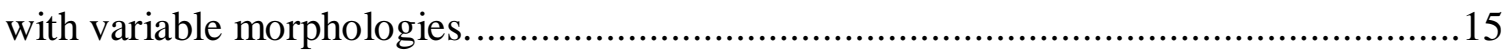

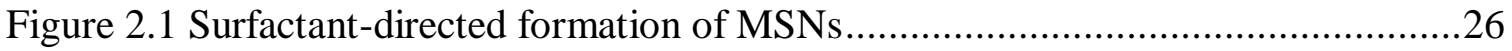
Figure 2.2 Ideal MSN-based host demonstrating appropriate target parameters for CEST-

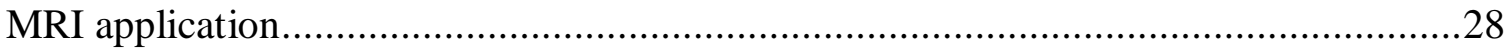

Figure 2.3 CTAB-based reaction scheme for the synthesis route of MSNs....................31

Figure 2.4 Infrared transmission spectra of A) CTAB, B) unetched MSNs, C) std-MSNs,

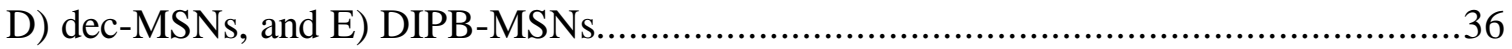

Figure 2.5 TEM images (Row 1), size TEM size distribution (Row 2), $\mathrm{N}_{2}$ sorption isotherms (Row 3), and corresponding BJH pore size distributions curves of std-MSNs,

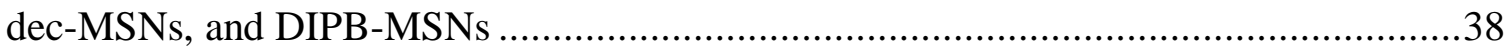

Figure 3.1 Schematic illustration for the template synthesis of hollow $\mathrm{SiO}_{2} \mathrm{NPs}$...........46 Figure 3.2 Ideal hMSN-based host demonstrating appropriate target parameters for CESTMRI application.

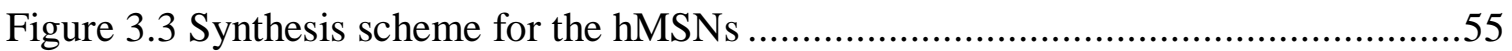

Figure 3.4 Schematic representation of the two different addition procedures (or reagent mixing procedures) explored in the synthesis of the solid $\mathrm{SiO}_{2}$ templates.....................57 Figure 3.5 TEM images of nanoparticles synthesized according to the reagent amounts presented in Table 3.1 .... .59

Figure 3.6 Supporting DLS size distributions of nanoparticles synthesized according to the

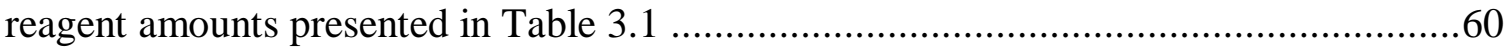

Figure 3.7 DLS results of solid $\mathrm{SiO}_{2}$ diameter as a function of $\mathrm{NH}_{4} \mathrm{OH}$ concentration....62 Figure 3.8 TEM images of A) Mesoporous shell coated Solid $\mathrm{SiO}_{2}$ nanoparticles B) Core etched mesoporous coated Solid $\mathrm{SiO}_{2}$ nanoparticles C) Nanoparticle with etching order reversed D) Infrared transmission spectra of $\mathrm{CTAB}$, mesoporous coated Solid $\mathrm{SiO}_{2}$ nanoparticles and complete hMSNs .....................................................................6 63

Figure 3.9 TEM image of complete hMSNs ....................................................65

Figure 3.10 Left) $\mathrm{N}_{2}$ porosimetry isotherm and Right) $\mathrm{BJH}$ pore size distribution curve for synthesized hMSNs

Figure 4.1 Effect of surface charge on the loading capacity of cationic vs. anionic dyes. 74 Figure 4.2 Thermogravimetric analysis (TGA) curves for std-MSNs and hMSNs .........76 Figure 4.3FTIR spectra of A) APTES, B) as-synthesized silica and amine modified std-

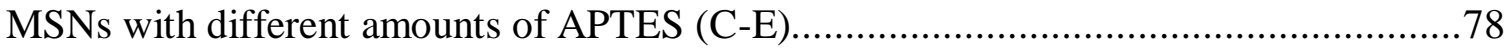


Figure $4.4 \mathrm{~N}_{2}$ porosimetry studies showing isotherms (Top) and pore size distribution (Bottom) of MSNs (Left column) and h-MSNs (Right).......................................79

Figure 4.5 Ninhydrin standard curve for amine concentration determination ..................80

Figure 4.6. Standard curve for determining the loading capacity ...............................82

Figure 4.7 TEM images of the different samples presented in Table 4.3 .......................84

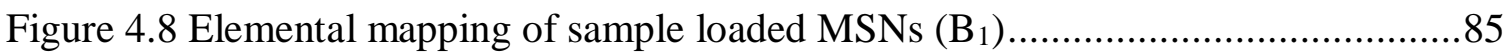

Figure 5.1 The sol-gel method is used to create $\mathrm{SiO}_{2} \mathrm{NPs}$ from tetraethoxysilane in aqueous ethanolic solutions.

Figure 5.2 Representative TEM images for Ludox-TM40 (A), larger $\mathrm{SiO}_{2}$ NP samples SS1 (B), SS2 (C), and SS3 (D), and a comparative TEM image from a non-seeded synthesis (E) under otherwise similar synthesis conditions

Figure 5.3 DLS distributions for SS1, SS2, and SS3 with modal sizes and PdIs. 100

Figure 5.4 TEM images (A) showing a thin mesoporous shell coated-SS1 with CTAB still in pores, (B) core-etched hybrid nanoparticles showing small rattle with a magnified image in the insert $(\mathrm{C})$ completely r-hMSNs after etching 103

Figure 5.5 Nitrogen porosimetry studies showing surface area and cumulative pore volumes for (A) Ludox-TM40, (B) Seeded SS1 and (C) Complete r-hMSNs and (D) Pore size distribution as determined by the JBH method). 105 
List of Abbreviations

CEST-Chemical Shift Exchange Saturation Transfer

CMC-Critical Micelles Concentration

CTAB-Cetyltrimethylammonium bromide

DEA-Diethanolamine

dec-MSN- Decane Mesoporous Silica Nanoparticle

DIPB-MSN- diisopropyl benzene Mesoporous Silica Nanoparticle

DMHA-N, N-dimethylhexadecylamine

EtOH-Ethanol

FDA- Food and Drug Administration

$\mathrm{H}_{2} \mathrm{O}-$ Water

h-MSN-Hollow Mesoporous Silica Nanoparticle

IUPAC - International Union of Pure and Applied Chemistry

LoD-Limit of detection

LE-Loading efficiency

MRI-Magnetic resonance imaging

MSN-Mesoporous Silica Nanoparticle

$\mathrm{NaOH}-$ Sodium hydroxide

$\mathrm{NH}_{4} \mathrm{OH}$-Ammonium hydroxide

NMR- Nuclear magnetic resonance

NPs-Nanoparticles

OTAB-Octyltrimethylammonium bromide

PARACEST-Paramagnetic Chemical Shift Exchange Saturation Transfer

PdI-Polydispersity Index

RF- Radiofrequency

r-MSN- Rattle mesoporous Silica Nanoparticle

SAR- Specific Absorption Rate 
$\mathrm{Si}(\mathrm{OR})_{4}(\mathrm{R}=$ various alkyl groups, usually Et or $\mathrm{Me})$,

$\mathrm{SiO}_{2}-$ Silica

std-MSN- standard Mesoporous Silica Nanoparticle

TEA-Triethanolamine

TIPB-Triisopropylbenzene

TOA-Trioctylamine 


\section{CHAPTER 1: INTRODUCTION TO CHEMICAL EXCHANGE SATURATION TRANSFER, ROLES OF NANOPARTICLES AND SILICA NANOPARTICLES}

\subsection{MRI AND CEST-MRI}

Magnetic resonance imaging (MRI) uses a strong external magnetic field, radio waves, and a computer to generate images. When exposed to a magnetic field, nuclei with an inherent magnetic moment populate low and high energy states according to the Boltzmann statistical probability distribution. The sum of all nuclear magnetic moments is the net magnetization $\left(\mathrm{M}_{0}\right)$, which is aligned along the longitudinal $(\mathrm{z})$ axis at equilibrium. A small excess of spins populates the lower energy. A radio frequency pulse at the resonance frequency of the nuclear spin applies torque to $\mathrm{M}_{0}$, coherently moving the net magnetization vector into the transverse plane $\left(\mathrm{M}_{\mathrm{XY}}\right)$. The net magnetic vector precesses about the external field at a radio frequency.

After the radio frequency pulse is turned off, the net magnetization vector precesses in the transverse plane. This precession gives rise to a current in the receiver coil, which is recorded and provides information specific to the chemical and magnetic environment of each chemically and magnetically distinct nucleus. In the case of MRI, spatial information is obtained by applying gradient fields that change the resonance frequency of the nuclear spins based on their location. MRI involves probing the differences detected amongst water molecule protons. Two-thirds of the human body is composed of water, allowing MRI to generate high-resolution images without utilizing ionizing radiation. This has made MRI the most popular NMR-based technique in diagnostics. ${ }^{1,2}$ The relatively low sensitivity of 
the techniques, which limits the detection of tiny lesions, has since been a limitation. Improving signal intensity has been the main objective of several studies. ${ }^{3}$

Using $T_{1}$ or $T_{2}$ contrast agents to change the nuclear magnetic relaxation rates constants results in increased or decreased signal intensity, depending on which effects are more evident (a basis for their classification). $\mathrm{T}_{1}$ or $\mathrm{T}_{2}$ contrast agents are employed to reach thermal equilibrium quickly to reduce the super long natural relaxation durations. $\mathrm{T}_{1}$ contrast agents are used in MRI to increase the difference in $\mathrm{T}_{1}$ relaxation rates by decreasing the $T_{1}$ rate constant of water protons directly near the contrast agent, whereas $\mathrm{T}_{2}$ contrast agents are used for decreasing the water signal intensity by shortening the transverse relaxation periods., ${ }^{4,5}$

All paramagnetic agents will cause both $\mathrm{T}_{1}$ and $\mathrm{T}_{2}$ to shorten, but because $\mathrm{T}_{2}$ in vivo is much shorter than $T_{1}$, an agent must significantly shorten $T_{2}$ for any observable effects to be realized. For this reason that $\mathrm{Mn}^{2+}$ and $\mathrm{Gd}^{3+}$ complexes have been used as $\mathrm{T}_{1}$ agents, and such paramagnetic complexes have been able to sense metabolite levels, $\mathrm{pH}$, redox, and oxygenation alterations in bulk tissue. ${ }^{6,7}$ Moreover, their low molecular weight enables rapid clearance through the renal system. However, sensitivity remains one of the hurdles justifying the small number of MRI agents making their way into living subjects.

Encapsulating many chelates into a single nanoparticle (NP) structure to increase local contrast agent concentration and thus increase signal intensity has been one strategy that has been used to reduce the high detection limits of molecular (or ionic) $\mathrm{T}_{1}$ complexes. ${ }^{8}$ Alternatively, using superparamagnetic metal oxide NPs generates greater $T_{2}$ 
contrast, which overshadows the smaller effects on $\mathrm{T}_{1}$, making them viable $\mathrm{T}_{2}$-shortening agents. Although it has been demonstrated that this class of agent has much lower detection limits than $\mathrm{T}_{1}$-shortening agents, the tendency of metal oxide NPs to remain intact means that they cannot be renally cleared (due to large size) and persist in the body well after administration. An additional major intrinsic limitation to both classes of agents is that they cannot be turned "off"; thus, one will need to acquire pre and post-injection images to determine signal changes, which might cause errors due to time delays and motion-induced artifacts..$^{9,10}$

Chemical Exchange Saturation Transfer (CEST) is an alternate MRI contrast enhancement mechanism that allows for the indirect detection of low concentration solute molecules (or CEST agents) with chemically distinct labile protons. ${ }^{11}$ Figure 1.1 below shows the two-pool proton exchange model that summarizes a CEST-based MRI mechanism. Exchangeable protons (in green) are saturated using a frequency-selective radiofrequency $(\mathrm{RF})$ pulse. The saturated spins are repetitively exchanged with the unsaturated spins (in blue) of bulk water protons via chemical exchange, thus decreasing the bulk water magnetization over time (CEST effects). CEST effects are quantified by comparing the water signal without saturation $\left(\mathrm{S}_{0}\right)$ to the water signal with saturation $\left(\mathrm{S}_{\mathrm{sat}}\right)$, where the magnitude of the decrease depends on the number of exchangeable protons on the molecule of interest and the exchange rate, $k_{s w}$. The repeated saturation and exchange of labile protons thus allows for the indirect visualization and quantification of otherwise undetectable low concentration molecules. Moreover, the ability of the pre-saturation pulse 
to be switched "on" and "off" allows pre and post-contrast images to be acquired at the same time, thus permitting direct comparison of images, reducing detection limits, and
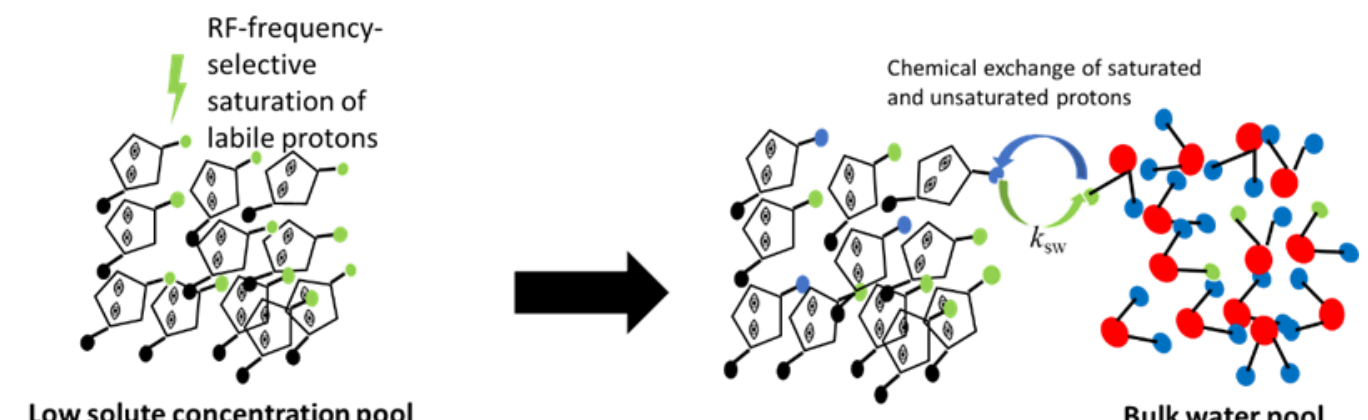

Low solute concentration poo

Bulk water pool

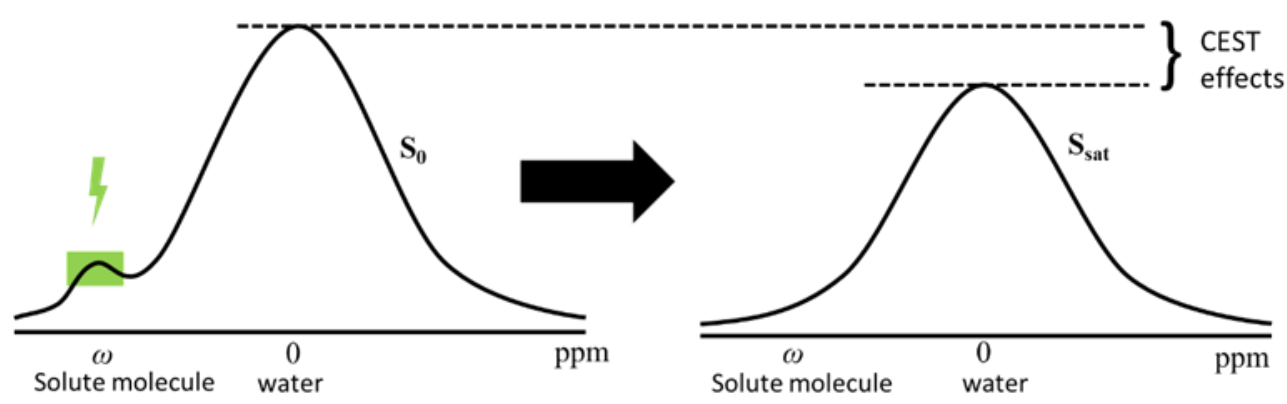

Figure 1.1 Chemical exchange saturation transfer: principles and measurements for pure exchange effects eliminating artifacts due to organ movement and repositioning; these features are an advantage over the conventional MRI technique.

CEST generation requires that a distinct chemical shift difference $(\Delta \omega)$ exist between the bulk water proton and the labile proton on the low concentration solute molecule, such that the proton exchange rate, $k_{\mathrm{sw}}$ is both relatively slow on the NMR time

$$
k_{s w}<\Delta \omega
$$

scale and less than $\Delta \omega$ (Equation 1.1). ${ }^{12,13}$ Naturally present functional groups on biomolecules have labile protons with chemical shifts distinct from water; for example, 
hydroxyl, amine, or amide groups on proteins thus can be used as CEST agents. ${ }^{10,14,15}$ However, because these functional group chemical shifts are relatively close to that of water, the slow exchange condition (Equation 1.1) implies that very slow exchange rates are required for these biological molecules if CEST effects are to be realized. In general, these molecules also have a relatively small number of exchanging protons per agent, causing their detection limits to be an order of magnitude higher than those of conventional $\mathrm{Gd}^{3+}$-based contrast agents. Furthermore, the proximity of some biomolecule functional group chemical shifts to that of water may result in indirect saturation of water molecules. These factors will contribute to a reduction in, or total loss of, CEST effects over time.

For CEST agents satisfying the slow to intermediate exchange rate condition, the observed CEST effects are approximately linearly correlated to the concentration of labile protons and dependent on the exchange rate. Thus, recent studies to improve the sensitivity of CEST have concentrated on increasing the numbers of exchanging protons ${ }^{15,16}$ and optimizing exchange rates. ${ }^{17,18}$ Increasing the number of labile protons allows the simultaneous saturation of many protons on the agent to transfer saturation to the bulk water pool, thus enhancing sensitivity. Increasing the proton exchange rate, such that saturation transfer from agent to water is more efficient, while still maintaining an exchange rate that is slow on the NMR time scale, increases the sensitivity by ensuring that more saturated spins are transferred to the solvent water (per unit time) thereby increasing the observed CEST effect.

Paramagnetic Chemical Shift Exchange Saturation Transfer (PARACEST) agents are 
paramagnetic ions that are chelated by organic multidentate ligands to create complex ions that possess a pool of labile protons and/or labile ligands, with large chemical shifts of 50 ppm or more ${ }^{12}$ compared to the typical 1-5 ppm of endogenous molecules. Because of these larger chemical shifts, CEST effects are realized even at more rapid chemical exchange rates ${ }^{14}$ without going beyond the intermediate exchange limit. ${ }^{19}$ While these relatively fast exchanging complexes can further be optimized to increase contrast, their dependence on the initiating saturation pulse means RF saturation intensities higher than FDA guidance limits (SAR whole-body exposure in patients with "normal thermoregulatory function" is $4.0 \mathrm{~W} / \mathrm{kg}$ and $1.5 \mathrm{~W} / \mathrm{kg}$ for all other cases) ${ }^{20}$ may be required, reducing the feasibility of applying such agents in vivo. The further development of paramagnetic systems that combine large chemical shifts with moderate-to-slow proton/water exchanges offers advantages of endogenous molecules and PARACEST agents. $^{14}$

Paramagnetic chemical shifts agents are designed to utilize water as the source of the saturated proton pool, and efforts have been made to construct agents with a slowexchanging water molecule bound to a coordination site of the paramagnetic complex. Because these systems utilize only one water coordination site per complex, and because the exchange rate is relatively slow, the sensitivity is low, as expected. This led researchers to the consideration of systems possessing larger numbers of water molecules in contact with the paramagnetic shift agents, for example, by encapsulation of a relatively fast exchanging shift agent within an isolated water pool, where the isolated pool is then in 
slow exchange with the bulk water pool (compartmental exchange) as conceptualized in Figure 1.2. For such systems, compartmental water exchange should be slow compared to the frequency difference between the two exchanging water pools, shifted inside and unshifted outside; otherwise, the resultant peak will be unresolved. However, the bulk

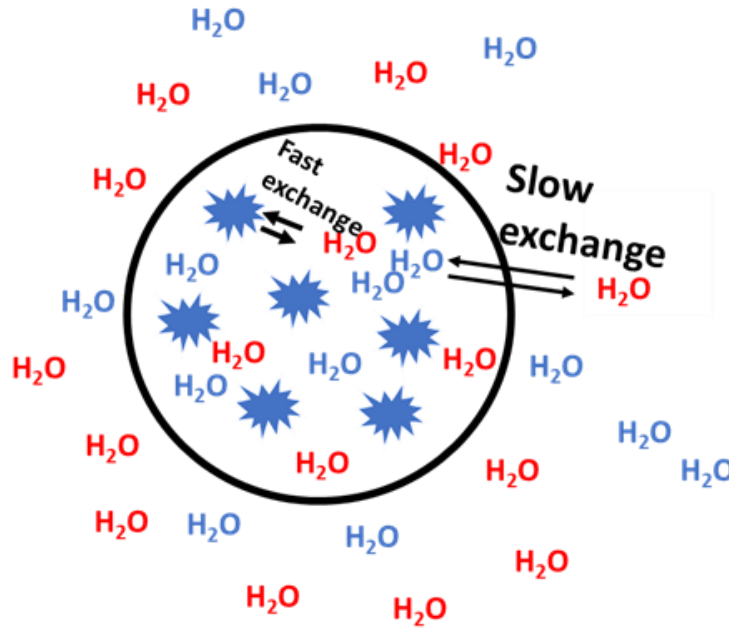

Figure 1.2 Mechanism for a nano-based host in CEST MR imaging

water's magnetization will not change considerably if the rate is too slow, and no contrast enhancement will be achieved. Different approaches have been explored to construct such compartmentalized paramagnetic agents to lower detection limits by increasing the local agent concentration (and thus the number of exchangeable sites) and optimizing the intercompartment water exchange rates.

\subsection{NANOPARTICLES IN CEST MR IMAGING}

Nanoparticles (NPs) of various types have shown great potential in biomedical applications 
due to attractive features such as prolonged blood circulation, ability to target a biological structure with surface-bound recognition elements, or to accomplish controlled release of a drug payload in the case of hollow or porous nanostructures; this has fueled the interest in developing functional, tailored NPs. ${ }^{21-23}$ Therefore, it is no surprise that several nanosystems, including dendrimers, supramolecular adducts, liposomes, micelles, and silica nanoparticles ( $\left.\mathrm{SiO}_{2} \mathrm{NPs}\right)$, have already been explored for potential use in CEST-MRI (Table 1.1).

In theory, these nano-based systems could increase CEST contrast by increasing the number of saturation sites or optimizing the exchange rate(s), or both. For example, Pikkermaat and co-workers developed functionalized poly(propylene imine) PARACEST dendrimers of various sizes (generations) and used them for CEST-based $\mathrm{pH}$ mapping. This work showed that the lowest concentration of agent detectable decreased as the number of amide protons increased; i.e., the limit of detection is lowest for the largest particles with the most amide groups. ${ }^{16}$ Some groups have also succeeded in increasing the number of saturation sites by making noncovalently bonded supramolecular adducts between a PARACEST agent and a macromolecule, where the PARACEST agent artificially shifts the resonance of a large number of labile protons on the macromolecule, allowing for selective irradiation of these protons and increasing the efficacy of saturation transfer. $^{24}$

Micelle-based CEST probes have also been developed and have shown great potential in lowering the detection limits of CEST-based MRI methods. For example, Evbuomwan and 
Table 1.1 Examples of nano-based systems that have been explored in CEST-MRI, advantages, and disadvantages

\begin{tabular}{|c|c|c|c|}
\hline Nanoparticle & Advantage & Disadvantages & References \\
\hline $\begin{array}{l}\text { Supramolecular } \\
\text { adducts }\end{array}$ & $\begin{array}{l}\text { Easy, cost-effective, and } \\
\text { facile approaches for } \\
\text { synthesis }\end{array}$ & $\begin{array}{l}\text { Possess a few numbers of exchangeable } \\
\text { protons. } \\
\text { Water kinetics difficult to tune } \\
\text { Linked with long-term toxicity effects }{ }^{25}\end{array}$ & $26-29$ \\
\hline Dendrimers & $\begin{array}{l}\text { Possess many } \\
\text { exchangeable protons. }\end{array}$ & $\begin{array}{l}\text { Incomplete safety profile, Unknown } \\
\text { biodegradation/excretion pathway, } \\
\text { Lacks well-understood surface chemistry } \\
\text { for functionalization, } \\
\text { Untunable water kinetics } \\
\text { Lack of reproducible standardized synthesis } \\
\text { methodologies }^{30}\end{array}$ & $16,31,32$ \\
\hline Micelles & $\begin{array}{l}\text { Possess a large interior } \\
\text { volume. } \\
\text { Generally regarded as } \\
\text { safe }\end{array}$ & $\begin{array}{l}\text { Poor loading capacity } \\
\text { Poor physical stability in vivo }\end{array}$ & $33-35$ \\
\hline Liposomes & $\begin{array}{l}\text { Possess a large interior } \\
\text { volume, } \\
\text { Safe, } \\
\text { Amenable } \\
\text { physicochemical }\end{array}$ & $\begin{array}{l}\text { Unstable } \\
\text { Costly and complex optimization process }\end{array}$ & $24,36-44$ \\
\hline $\begin{array}{l}\text { Mesoporous } \\
\text { silica }\end{array}$ & $\begin{array}{l}\text { Large surface area, } \\
\text { Generally regarded as } \\
\text { safe, } \\
\text { Easy, cost-effective, and } \\
\text { facile approaches for } \\
\text { synthesis }\end{array}$ & $\begin{array}{l}\text { Limited interior volume } \\
\text { Water kinetics difficult to optimize }\end{array}$ & $14,43,45$ \\
\hline
\end{tabular}

co-workers developed some lanthanide-tetraamide complexes with variable alkyl-carbon chain lengths and incorporated them during micelle formation; the different alkyl-carbon chain lengths of the complexes were observed to result in different-sized micelles and directly impact the measured water residence lifetimes in the micelles. For those micellar Ln-tetraamide agents exhibiting slow to medium water exchange kinetics, there was a noted increase in CEST sensitivity with longer alkyl chains Ln-complexes incorporated in 
the micelles compared to the monomethylamide control sample (5.3 $\mu \mathrm{m}$ for the $\mathrm{C}_{16}$ analog vs. $1.3 \mathrm{mM}$ for monomethyl-Ln-micelle). ${ }^{9}$

Overall, for nano-based CEST agents with paramagnetic complexes encapsulated in organic vehicles, while they can possess comparatively more exchangeable protons versus a single molecular agent, they still have low sensitivities that are mainly in the mM range. ${ }^{46}$ It can be argued that even better enhancement can be realized with fine control of the water exchange rate (kinetics) between the two water pools.

A system designed to both increase the exchanging proton population and optimize the exchange rate can be hypothesized to offer superior CEST efficacy. One way to envision accomplishing this is a liposome structure encapsulating a paramagnetic lanthanide complex (lipoCEST) in contact with a high volume of intraliposomal water molecules. Therefore, it is no surprise that after the first development and demonstration of the potential use of a lipoCEST agent by Aime and co-workers, ${ }^{24}$ more research efforts on liposome-based CEST agents would follow. Some lipoCEST agents displayed tremendous sensitivity, with the detection limit for the best agent reported to be in the picomolar concentration range. ${ }^{36-41,43,44,47}$

In a lipoCEST agent, the lanthanide complex trapped inside the liposome shifts the resonance frequency of the trapped water pool, enabling it to be selectively pre-saturated. The water-permeable phospholipid membrane of the liposome allows for water exchange that can be fine-tuned to the desired slow exchange regime by changing the lipid composition of the membrane. The size of the liposomes can also be varied (50 to 
450nm), ${ }^{48}$ and smaller liposomes have shown increased CEST effects due to their large surface-to-volume ratio offering an ample membrane water exchange. ${ }^{42}$ Despite the potential of lipid-based systems due to a large proton population in slow exchange, the instability of most liposome-based agents in vivo potentially limits their application in imaging. Further, although the exchange rate across the nanoscale host is potentially modifiable via incorporating specialty phospholipids, ${ }^{40}$ high cost and synthetic difficulty issues may hamper clinical translation. Therefore, there is a need to develop nano-based CEST systems that are stable, biocompatible, show sub-nanomolar sensitivities, and have exceptional amenability for potential functionalization at low costs.

\subsection{MESOPOROUS SILICA AS SUITABLE CEST-MRI AGENT HOST}

Mesoporous silica nanoparticles (MSNs) with their varied morphologies, uniform mesopores, and large surface areas are considered synthetically accessible, biocompatible, and relatively stable hosts for the delivery of drugs in biomedicine; a steady increase in publications on both preparations and applications of different silica-based nanomaterials has been noted in the last decade. ${ }^{45,47}$ The publication surge has resulted in welldocumented synthesis methods designed to scale up material production, fine-tune porosity, surface area, and volume, and tailor the surface chemical functionality for different applications. ${ }^{49-51}$ The presence of open channels and high surface area has paved the way for MSNs to be examined as possible MRI contrast agents, since $\mathrm{T}_{1}$ agents, like gadolinium chelates or paramagnetic ions, can be confined on the surface or inside the pores, thus localizing high concentrations of the agents and increasing the sensitivity of 
traditional $\mathrm{T}_{1}$ MRI techniques. ${ }^{45}$ The success of MSNs in conventional MRI techniques ${ }^{52,53}$ has led to their examination as CEST-MRI agents with great initial promise. , $^{9,43,45}$

By anchoring lanthanide chelates onto the surface of MSNs, Ferrauto and colleagues were able to effectively shift the surface silanol protons resulting in the marked improvement in sensitivity (with a limit of detection (LoD) in the $\mu \mathrm{M}$ range) in comparison to control samples with free chelates. ${ }^{43}$ Recently, Carniato and group further demonstrated the feasibility of mesoporous silica-based systems in CEST-MRI by anchoring lanthanide chelates onto the surface of MSNs and studying the interaction between the bound chelate and bulk water. They demonstrated that these systems exhibit slow water kinetics inside pores which is essential for improving their CEST efficiency. The developed complexes reached excellent sensitivities of $55 \pm 5 \mu \mathrm{M}$. Despite these recent systems exhibiting higher sensitivity than dendrimers and micelles, MSN systems so far are still inferior in achieving the sensitivity of the best lipoCEST agents, which have been indicated to reach nanomolar levels. $^{46}$

We hypothesize that this could be due to lower, or more constricted, internal volumes accessible to the (anchored) chelates within the MSNs compared to the internal volume of liposomes. Further, surface anchoring of the lanthanide chelates to the MSN surfaces is likely not ideal, compared to internalizing them, due to the lack of compartmentalizing barrier that could regulate the water exchange kinetics. With the optimization of surface chemistry and NP internal architecture (i.e., arrangement of the host material and void space in three dimensions), silica nanoparticles have the potential to become scalably 
synthesized, biologically stable hosts suitable for applications in vivo CEST-MRI. Furthermore, a silica-based host that is hollow can offer comparable volume to that of the lipoCEST agents, thus further increasing silica-based systems' sensitivity to levels comparable to those of lipoCEST agents.

(A)

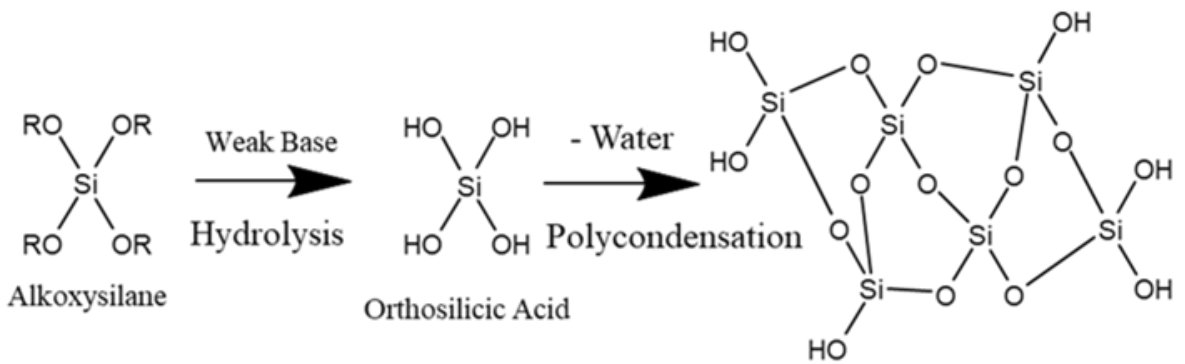

Where $\mathrm{R}$ is an alkyl group

Silica
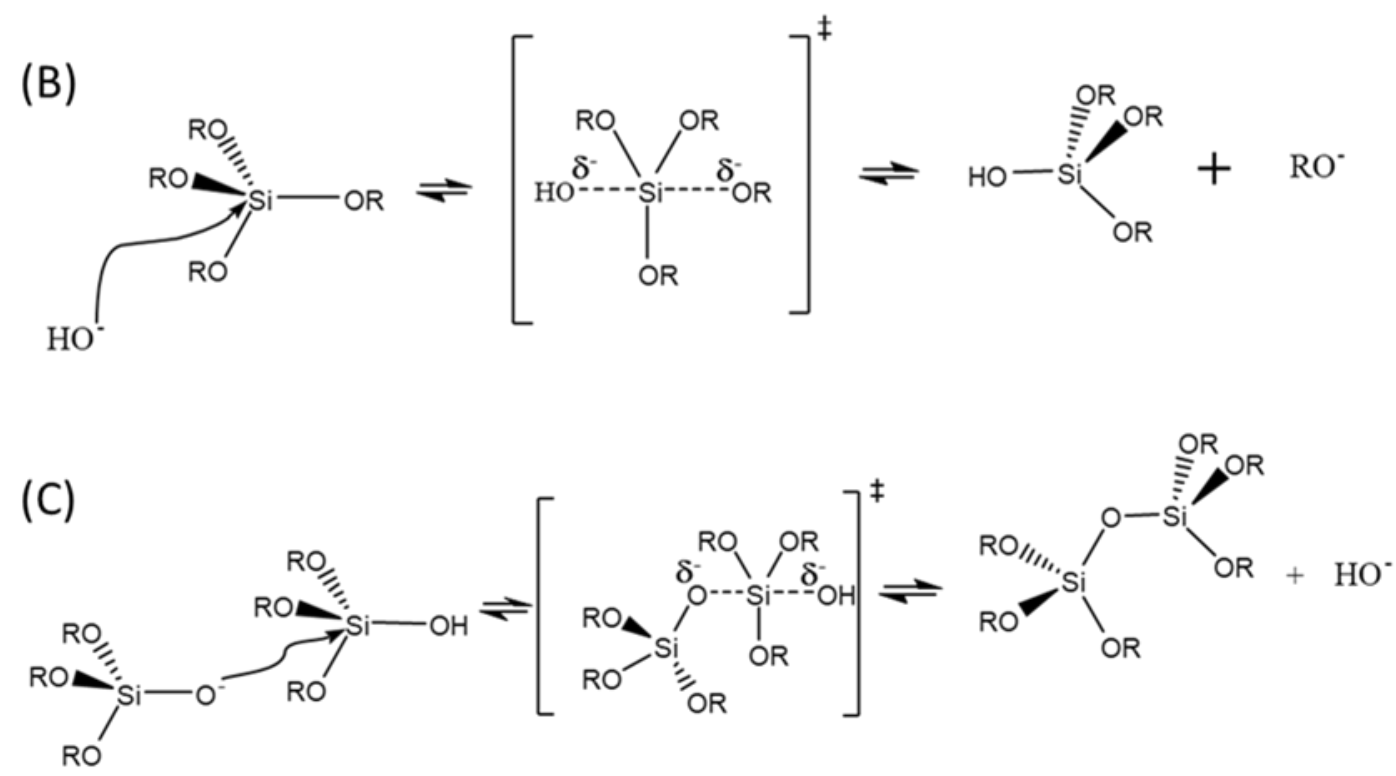

Figure 1.3 The sol-gel method for making $\mathrm{SiO}_{2} \mathrm{NPs}$, here from tetraethoxysilane in aqueous ethanolic solutions. During hydrolysis, the alkoxysilane undergoes hydrolysis with silanol groups replacing alkoxy groups in a nucleophilic substitution reaction. This is followed by a condensation reaction where neighboring silanols react, giving off water, thus forming siloxane bridges holding together the silica

There are various methods for synthesizing $\mathrm{SiO}_{2} \mathrm{NPs}$ with various morphologies, primarily 
based on the Stöber method, a sol-gel process. This wet chemistry synthetic approach involves hydrolysis and condensation of alkoxysilane monomers in the presence of an acid or base catalyst to produce nanoparticles having various sizes, depending mainly on the relative concentrations of the starting reactants. ${ }^{54}$ Figure 1.3 gives an overview of the basecatalyzed sol-gel (A) and mechanisms of hydrolysis (B) and condensation (C). Hydrolysis causes an alkoxy group to be substituted for a hydroxyl, forming a pentacoordinate transition state (Figure 1.3B). Multiple alkoxy groups may be hydrolyzed depending on the $\mathrm{Si} / \mathrm{H}_{2} \mathrm{O}$ ratio. The rate of each hydrolysis step is determined by the stability of the transition state, which is determined by the relative electron-withdrawing or electron-donating power of $-\mathrm{OH}$ and $-\mathrm{OR}$ groups. As a result, subsequent hydrolysis stages become increasingly faster under basic conditions. As illustrated in Figure 1.3C, condensation follows a similar process, culminating in siloxane linkages and the formation of a silica network.

The precise control of $\mathrm{SiO}_{2}$ NPs' morphology, size, uniformity, and dispersity is a progressive research concern due to their significant potential in scientific and technological applications. There have been several studies in which the Stöber method's kinetics and mechanisms have been investigated. ${ }^{55-57}$ This has led to a rational framework for the synthesis methods controlling size and morphology. During synthesis, the formation of particles and their properties rely on the balance between nucleation and growth, the two interdependent and highly dependent physicochemical parameters ${ }^{55,58}$ affecting the quality of particles. The precise control of starting material concentration and conditions is required to create an environment that facilitates burst nucleation and avoids multiple 
nucleation events, ${ }^{59}$ which may widen the size distribution of the particles.

MSNs with a variety of different morphologies have so far been synthesized. Figure 1.4 summarizes examples of different types of $\mathrm{SiO}_{2} \mathrm{NPs}$ that can be obtained by utilizing the central chemical principles of the Stöber method. MSNs can be synthesized by modifying the Stöber method to include a surfactant, a template for introducing pore into the silica

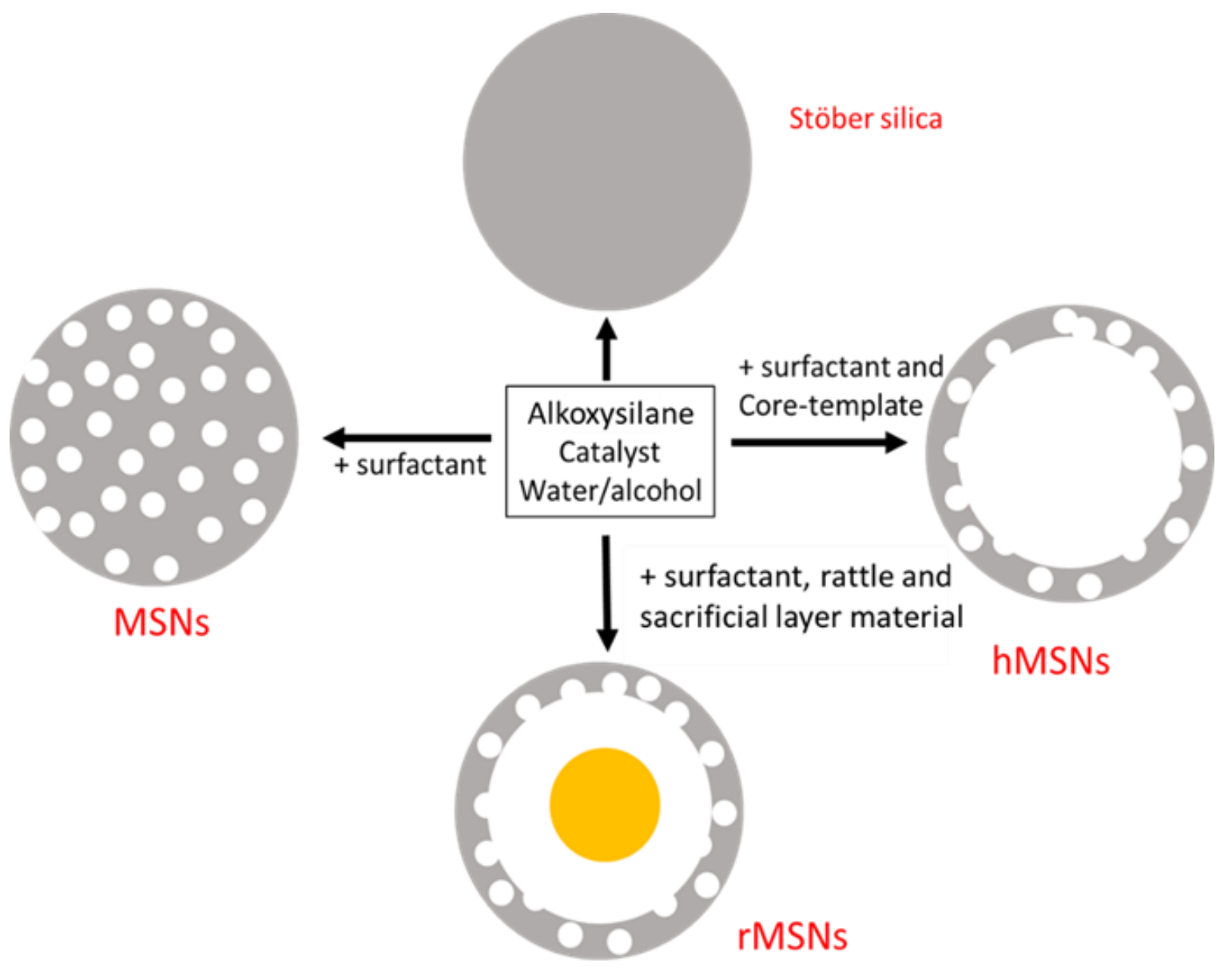

Figure 1.4 Synthetic routes based on the Stöber method for making different SiO2 NPs with variable morphologies. The introduction of surfactants into the solution of the starting material followed by the subsequent removal by chemical etching or calcination constitutes the basis of the synthesis of MSNs. hMSN are formed by the selective etching of a carefully chosen template resulting in a large cavity within the NP. In some cases, functional inorganic nanocrystals are also incorporated with a sacrificial material which, when dissolved, leaves the inorganic material within the cavity ( $r$-hMSNs) 
network. When added to an aqueous solution, an amphiphilic surfactant like cetyltrimethylammonium bromide (CTAB) forms spherical micelles. The outer, hydrophilic surfaces serve as small nucleation centers. The hydrolyzed silica precursors (alkoxy- or hydroxysilane) are concentrated and condensed to form an amorphous silica layer around the micelle. Following the synthesis and isolation of the surfactant/silica complex, the surfactant can be removed using either an ethanolic acid etch or by calcination in air, leaving behind mesopores (IUPAC definition of mesopore sizes: 2-50 nm) within the condensed silica nanostructure.

The synthesis of hollow mesoporous silica nanoparticles (hMSNs) includes adding a solid template to the aqueous surfactant/alkoxysilane mixture, which leaves behind a void interior when selectively removed. As in MSNs synthesis, the surfactant forms spherical micelles in the solution before initiation of the reaction by base hydrolysis. The hydrophilic surface of the micelles serves as small nucleation centers by concentrating alkoxy- or hydroxysilane at the hydrophilic interface, eventually resulting in the formation of an amorphous silica layer surrounding the entire micelle. The formed silica/surfactant structures are then deposited onto the solid template. When subsequently removed by selective structural etching or chemical dissolution (depending on core material composition) results in a hollow cavity. ${ }^{60-62}$ Just like in the synthesis of MSNs, the surfactant is finally removed by either calcination or acid etching. Closely related to hMSNs are rattle-type hMSNs (r-hMSNs), resulting from the template's partial etching. Given the range in viable $\mathrm{SiO}_{2} \mathrm{NP}$ host morphologies that are synthetically accessible using 
the famous Stöber technique, $\mathrm{SiO}_{2}$ NPs present limitless opportunities for obtaining morphological features and optimizing surface treatment to achieve high payloads of agent/water and optimized inter-compartmental water exchange kinetics, respectively.

\subsection{SCOPE OF THIS WORK}

Given the consistent development of $\mathrm{SiO}_{2}$ NPs for MRI and many other applications, due to their malleable morphology, tunable void volumes, and ease of chemical surface modifications, this thesis outlines the synthesis of two MSN types as possible hosts for paramagnetic chemical shift agents. Our overall goal is to prepare chemical shift agentloaded MSN hosts coated by a water-permeable $\mathrm{SiO}_{2}$ layer, to achieve the low detection limit in CEST-MRI. To do this, we will need two conditions to be met by the $\mathrm{SiO}_{2}$-based CEST agent: 1) the amount of encapsulated contrast agent and water (per particle) should be high, and 2) we will need to apply a coating that prevents the chelate from leaking out, while the encapsulated water can still exchange with the exterior water at least an appreciable rate (which could be further optimized). The two platforms will be pursued simultaneously as it is hard to predict whether MSNs' well-documented, easy synthesis route or hMSNs' independent volume and porosity optimization will be superior in maintaining two water pools that exchange at slow rates NMR time scales.

Our overarching hypothesis is that the morphological arrangement of the void volume/silica network and the surface coating characteristics (e.g., thickness, density, hydrophilicity) are the two most important parameters that can be optimized to increase 
CEST effects by ensuring an optimum payload and exchange rate of small molecules between the NP interior and exterior, respectively.

Overall, a thin silica layer coating is expected to be water permeable to some extent, allowing for water exchange between NP interior and exterior. While typical Stöber silica synthesis products are usually considered non-porous, many studies have concluded that chains of the solid network silica comprise pores that water molecules can penetrate. ${ }^{63-66}$ Further, evidence of gold particles embedded in solid silica being leached out over time supports a level of porosity within the Stöber-type silicas, as do findings that show that the experimentally determined number of hydroxyl groups consistently exceeds the theoretically calculated value based on the external surface area. ${ }^{64,67}$ Bazula and colleagues believe the degree of porosity depends on the specific synthesis parameters. The hydrophilic nature of the surfaces allows water to flow in and out of the solid. ${ }^{63}$ Our modified synthesis for hMSN also suppose a level of permeability as indicated by selective structural etching of the core while the coating remains intact.

This thesis project aims to establish synthetic access to two MSNs with different internal morphologies and determine whether a solid silica coating can effectively encapsulate the charged paramagnetic contrast agents within the MSNs interiors. With evidence of solid silica being porous to water, water can pass in and out of our host using a silica coating. At the same time, large molecules like a lanthanide complex would not escape, thus effectively establishing and maintaining a two-pool slow-rate exchange system required for CESTbased MR imaging. Accomplishing these goals will advance our knowledge of how the 
morphological and surface chemical features can be optimized to achieve high internal payload concentration and assist in identifying a lead MSN host candidate with which to optimize the small molecule exchange rate between interior and exterior compartments in further work. The thesis also presents two novel synthesis procedures for making monodisperse solid silica NPs and r-hMSNs.

Chapter 2 describes the synthesis of MSNs. MSNs have demonstrated immense potential as nanocarriers in MRI, offering plenty of surface area onto which gadolinium chelates have been anchored, resulting in increased relaxivities of the respective chelates. The synthesis procedure described in this thesis is based on a modified literature protocol that replaces the traditional sodium hydroxide with triethanolamine as the catalyst. Details of how the incorporation of two swelling agents (including one not reported previously) is also presented. The chapter concludes with the presentation of the different analytical techniques that have been used to characterize the synthesized NPs.

Chapter 3 outlines the synthesis of hMSNs. hMSNs have, in addition to the large surface area rendered by the mesoporous shell, a huge void space in the middle that can hold increased amounts of guest molecules. For this reason, hMSN may act as a better host as they present an opportunity for optimizing the chelate quantity. A modified literature-based method has been used, which has three steps: solid core synthesis, mesoporous shell deposition, and etching. A delayed-addition procedure for synthesizing the template was also developed. The chapter concludes with the presentation of the different analytical techniques that have been used to characterize the synthesized NPs. 
Chapter 4 describes the procedure for loading, coating, and accessing the effectiveness of the NPs to keep the load from leaking. For effective loading, the surface of the particles is rendered positively charged through amination, followed by electrostatic attractionassisted loading. Coating the loaded particles with a silica layer ensured the trapping of the load inside the pores. Details of functionalization, loading, coating and assessment of coating effectiveness are well presented and aided by different characterization techniques.

Chapter 5 describes developing a novel reproducible silica synthesis technique for producing uniform-sized solid $\mathrm{SiO}_{2}$ NPs particles, the most studied silica (synthesis and application). In this Chapter, a process is advanced to use the plentiful, reasonably-priced small $(22 \mathrm{~nm}) \mathrm{SiO}_{2}$ NPs as seeds in a Stöber synthesis to translate their monodispersity to large particle sizes ( $\sim 85-360 \mathrm{~nm})$. The resultant solid NPs were then used to synthesize $\mathrm{r}$ hMSNs. Like hMSNs, they possess an enormous surface area rendered by the presence of the mesoporous shell, huge void space in the middle that can keep improved quantities of guest molecules, and small rattle debris made of different metals or other inorganic compounds. Varying the selection of rattle and having specific surface functionalization within the interior can make the application of these silica NPs more significant to multi applications. The procedure to use seeded Stöber silica in synthesizing r-hMSNs, including the characterization techniques utilized, has been presented.

Chapter 6 concludes the findings of Chapter 2, Chapter 3, Chapter 4, and Chapter 5. The Chapter suggested how gained knowledge can be furthered to propel the use of silica NPs in CEST-MRI and also give insights on the potential use of r-hMSNs 
While the author designed all the experimental synthesis work for this thesis, some of the characterizations were done by other groups or collaborated with other groups. The author completed data analysis afterward. Kevin Fabrizio conducted nitrogen porosimetry studies (Brozek Lab, University of Oregon). Abdul Emmanuel (Rananavare Lab, Portland State University assisted with the thermogravimetric data acquisition). During the author's training, Dr. H. Winter (Goforth Lab, Portland State University) contributed to most of the photographs in Chapters 2 and 3. The author mentored and worked with four undergraduate students who helped with various project areas during the research. In Chapter 2, the author worked with Anne Wachana to manufacture mesoporous silica nanoparticles and optimize the amine modification technique. For the preliminary dye-loading experiments in Chapter 4, the author worked with Justin Pena. In Chapter 5, the author worked with Dimitri Buckallew to create rattle mesoporous silica nanoparticles. Sophia Kim statistically analyzed the different batches of produced nanoparticles. 


\section{CHAPTER 2: SYNTHESIS OF MESOPOROUS SILICA NANOPARTICLES (MSN)}

\section{$2.1 \quad$ INTRODUCTION}

MSNs offer many advantages as hosts for therapeutic and diagnostic molecules, including contrast agents; thus, their synthesis optimization remains a top research interest. Through careful $\mathrm{pH}$ control, different templates and solvents, hydrolysis rate control, MSNs with different morphologies can be synthesized. The synthesis of MSNs will be discussed in detail by looking at the roles of different starting reagents and mechanisms of formation.

\subsubsection{Synthesis of MSNs}

Despite the emergence of alternative synthesis methods like the precipitation method ${ }^{68}$ and the microemulsion method, ${ }^{69}$ the Stöber process continues to be the most applied and studied synthesis procedure due to its simplicity, effectiveness for morphological control, and ease of scale-up. ${ }^{70}$ As already presented in Figure 1.3, the Stöber process generally involves using a weak base that catalyzes the hydrolysis of an alkoxysilane to form orthosilicic acid. Polycondensation of the orthosilicic acid then occurs (via adjacent hydroxyls), resulting in the formation of siloxane bridges that hold together the silica network.

Following an in-depth understanding of the Stöber-based NP synthesis mechanism for producing solid $\mathrm{SiO}_{2} \mathrm{NPs}$, several modifications have been made, resulting in significant improvement in size and polydispersity control and tailoring other morphological characteristics. One such modification to the procedure was the inclusion of surfactants to 
introduce porosity into the $\mathrm{SiO}_{2}$ network. ${ }^{71}$ Further modifications followed in which careful selection of surfactant, the selection of catalysts, the tuning of reagent ratios, concentrations, and synthesis $\mathrm{pH}$, and use of organic silanes allowed for the synthesis of MSNs of desirable size, morphology, and pore distribution. ${ }^{72-75}$ Table 2.1 shows examples of primary starting reagents and a summary of their roles in determining the final MSNs characteristics, further discussed below.

Table 2.1. Summary of MSNs morphological characteristics based on starting materials, and their role in morphological effects ${ }^{76-78}$

\begin{tabular}{|c|c|c|}
\hline Possible Reagents & Examples & Morphological effects \\
\hline $\begin{array}{l}\text { Surfactants/ } \\
\text { structure directing } \\
\text { agents }\end{array}$ & $\begin{array}{l}\text { CTAB, CTAC, Triton X-100, Tween } \\
\text { 20, 40, 60, 80, OTAB } \\
\text { Pluronic F123, F127 }\end{array}$ & $\begin{array}{l}\text { Mesostructure (disordered, wormhole- } \\
\text { like pores, hexagonal network silica) } \\
\text { Pore volume and size changes } \\
\text { Hierarchical structures can be observed } \\
\text { (tubes, helical fibers) }\end{array}$ \\
\hline $\begin{array}{l}\text { Pore swelling } \\
\text { agents }\end{array}$ & TIPB, TOA, decane, or DMHA & Enlargement of pore size \\
\hline Silica sources & $\begin{array}{l}\mathrm{Si}(\mathrm{OR})_{4}(\mathrm{R}=\text { various alkyl groups, } \\
\text { usually Et or Me), } \\
\mathrm{Si}\left(\mathrm{OC}_{3} \mathrm{H}_{3}\right)_{3} \mathrm{C}_{2} \mathrm{H}_{3} \\
\mathrm{Na}_{2} \mathrm{SiO}_{3}\end{array}$ & Nanoparticle size, charge \\
\hline Cosolvent & EtOH, heptane & Solubilization of TEOS \\
\hline Base catalysts & TEA, $\mathrm{NaOH}, \mathrm{NH}_{4} \mathrm{OH}, \mathrm{DEA}$ & $\begin{array}{l}\text { Affects nanoparticle size by altering } \\
\text { hydrolysis (primarily affecting } \\
\text { nucleation) and condensation rates } \\
\text { (primarily affecting growth) }\end{array}$ \\
\hline
\end{tabular}

Surfactant chain length and the effective radius of the head group determine micelle shape (for example, spherical, hexagonal columnar, cubic columnar, or lamellar); thus, the 
appropriate surfactant can be intentionally chosen to template the desired $\mathrm{SiO}_{2}$ mesostructure. ${ }^{73,79,80}$ The surfactant concentration can also be controlled to achieve desirable porosity (pore volume) within the silica network ${ }^{80}$ but only over a limited range, with pore sizes usually ranging from 1.8 to $2.8 \mathrm{~nm}^{77}$ across a vast number of surfactants. Such pore diameters may be small for significantly large biomolecule passage. However, many small molecules are expected to be penetrable (such as water, $2.8 \AA$ ).${ }^{81}$ Incorporating additional hydrophobic molecules (swelling agents) into the reaction mixture increases pore size by enlarging the volumes of the templating micelles to create larger pores. Swelling agents like tri-isopropylbenzene (TIPB), tri-octylamine (TOA), decane, or N, Ndimethylhexadecylamine (DMHA) have been used in past works. ${ }^{79,82,83}$

Different silica sources have been used in MSN synthesis. Their effects on resulting nanoparticle size, either in isolation or in combination with other factors, have been well studied; tetraalkoxysilanes $\left(\mathrm{Si}(\mathrm{OR})_{4}\right)$ are the most commonly used precursors. To highlight a representative size-related finding, Yamada et al. synthesized MSNs ranging in size from 20 - $80 \mathrm{~nm}$ using $\mathrm{Si}(\mathrm{OR})_{4}$, where $\mathrm{R}=\mathrm{Me}$, Et, $\mathrm{Pr}$, and $\mathrm{Bu}$. The results showed that the alkoxysilane with the shortest chain $(\mathrm{R}=\mathrm{Me})$ produced nanoparticles with the smallest diameters. The overall size decrease noted for smaller alkyl groups was attributed to a faster intrinsic hydrolysis rate, resulting in nucleation dominating particle growth. ${ }^{84}$ Furthermore, the surfactant/alkoxysilane ratio can be adjusted to control pore structure in MSNs effectively. In one study, the surfactant/alkoxysilane molar ratio was increased from 0.5 to 2.0 , and the range of exhibited morphologies was hexagonal, cubic, and lamellar. ${ }^{85}$ 
Although MSNs can be synthesized under mildly acidic conditions, ${ }^{86,87}$ ammonia and other organic amines continue to be catalysts of choice in the synthesis of MSNs. Such bases catalyze the silica precursor hydrolysis and further alter the hydrolysis and condensation rates, which can effectively control the mean size of the particles. ${ }^{79}$ For example, one study that used $\mathrm{Na}_{2} \mathrm{HPO}_{4}-\mathrm{NaH}_{2} \mathrm{PO}_{4}$ as the catalyst in an $\mathrm{EtOH} / \mathrm{H}_{2} \mathrm{O}$ media showed that the particle size increased with the solution's starting $\mathrm{pH}(6-10) .{ }^{88}$ When L-lysine was used as a base catalyst, it inhibited the growth of silica particles after nucleation. This decrease in the sizes of the particles was attributed to a decrease in condensation rates caused by electrostatic interactions between protonated L-lysine ammonium groups and negatively charged silanol groups on the forming silica surfaces. ${ }^{89}$ Moreover, Moller et al. also demonstrated that a catalyst like TEA could act as a complexing agent for silicate species, thus inhibiting the growth of mesoporous particles and ensuring the production of nonaggregated nanoparticles. ${ }^{90}$

\subsubsection{Mechanism of MSNs synthesis}

Despite the existence of different proposed mechanisms depending on starting materials, the liquid-crystal templating (LCT) mechanism, a mechanism similar to the classical nucleation theory $(\mathrm{CNT})$, remains the most popular and agreed-upon mechanism for synthesis of mesoporous $\mathrm{SiO}_{2} \mathrm{NPs} ;{ }^{91,92}$ however, in MSN formation, nucleation starts with the surfactant micelle template. The LCT mechanism for MSN synthesis has two main stages: (i) the surfactant molecules self-assemble during micelle formation, which occurs above the critical micellar concentration (CMC) and ultimately results in the establishment 


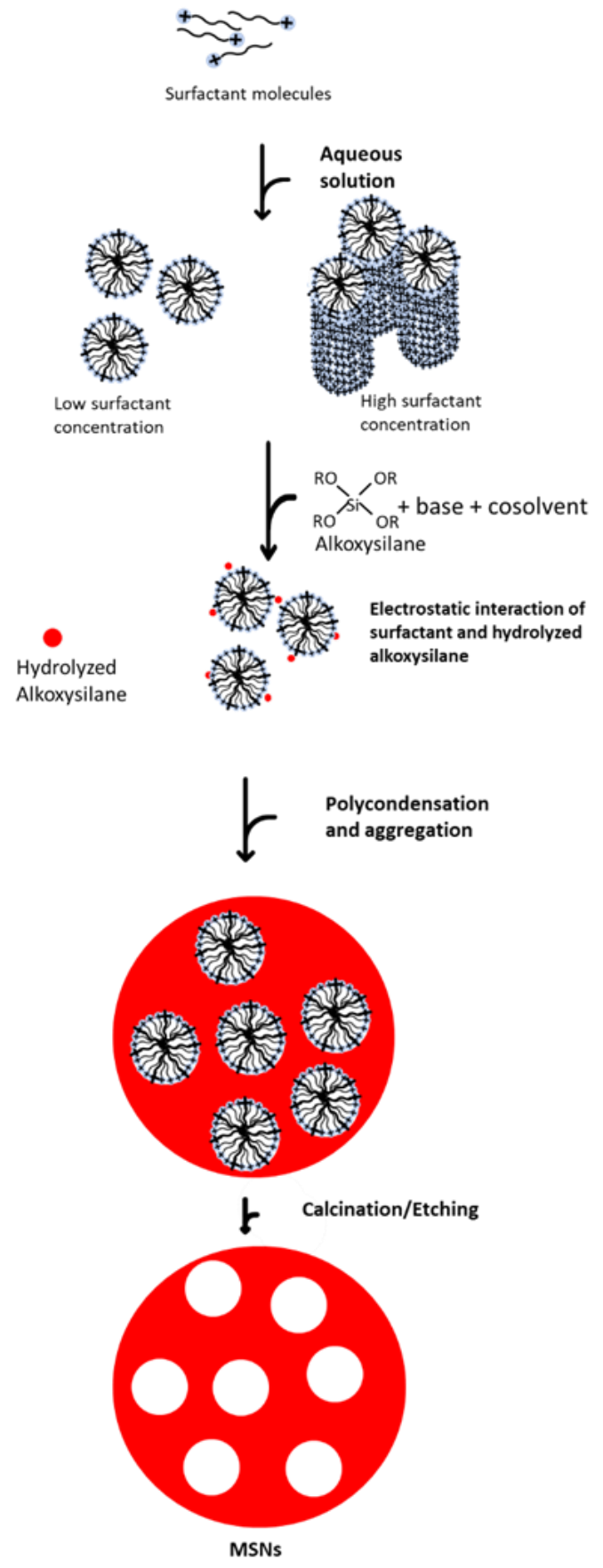

Figure 2.1 Surfactant-directed formation of MSNs (Adapted from Narayan et al., 2018) ${ }^{79}$ 
of an ordered structure that functions as a template; and (ii) the alkoxysilane is hydrolyzed into highly reactive monomers that subsequently undergo polycondensation and form the silica network surrounding the organic template. ${ }^{93}$ Figure 2.1 illustrates the LCT mechanism for MSN synthesis, where a cationic surfactant is used as an example. The interaction of the solvent with the cationic surfactant causes the cooperative self-assembly of the surfactant species when its concentration is higher than CMC. Depending on whether the concentration is high or low, the resultant micelles can be spherical or tubular. Under alkaline conditions, the alkoxysilanes are hydrolyzed, and they arrange themselves to the surfactant micelle through electrostatic interaction of their deprotonated silanol groups and cationic heads of the micelles. The formed micelle-silica template then aggregates and, following proper aging, results in silica-micelle composite nanoparticles of specified sizes. To give rise to mesoporous silica, the surfactant is removed by either calcination or solvent extraction. ${ }^{77,91}$

\subsubsection{Motivation and scope}

MSNs have been used in MRI due to their adjustable pore diameter, high chemical and thermal stabilities, large surface areas, excellent biodegradability, and high biocompatibility. However, MSN synthesis protocols are hampered by batch-to-batch inconsistencies, which limits potential application studies due to inconsistencies in physical-chemical properties. Thus, robust and reproducible methods must be developed to adjust synthetic parameters that yield MSNs with desired sizes, pore diameters, and morphological features. This chapter aims to make MSNs with the desirable properties 
shown in Figure 2.2 for use in subsequent studies using a modified literature procedure and then quantitatively assess method reproducibility and viability in pore expansion.

Nanoparticle dimensions of $100-200 \mathrm{~nm}$ are the best choice for preventing fast release, the acute toxic effect, and aggregation in physiological fluids, blood capillaries, and

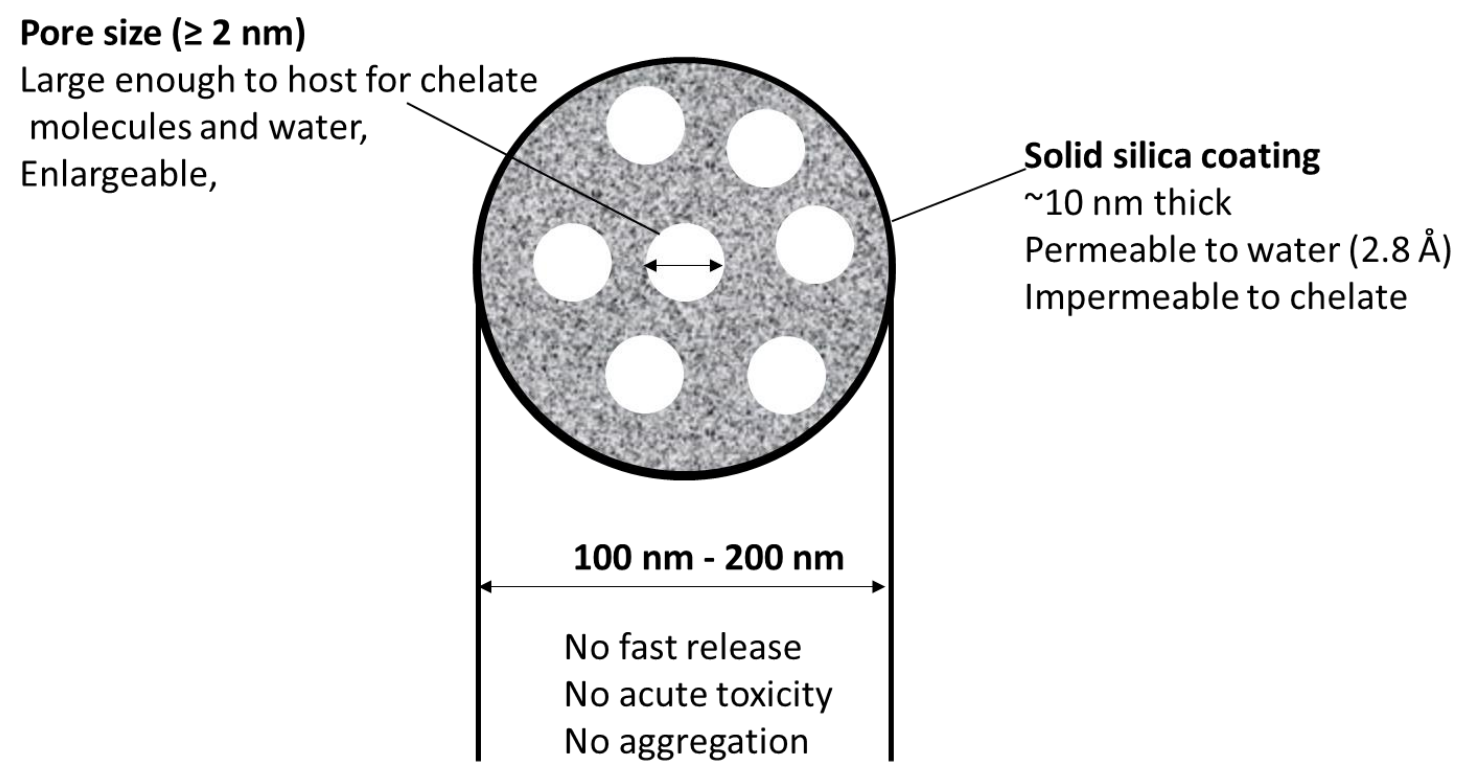

Figure 2.2 Ideal MSN-based host demonstrating appropriate target parameters for CEST-MRI application alveoli. To allow for the deposition of a thin silica shell $(10 \mathrm{~nm})$ that effectively traps the chelate inside, an approximate size of $80 \mathrm{~nm}$ was chosen while remaining within size limits and optimizing the surface area to volume ratio for loading. ${ }^{94}$ Pore diameters greater than $2 \mathrm{~nm}$ are large enough to allow chelates and smaller water molecules to pass through. If the pores are small, adding more hydrophobic molecules (swelling agents) to the reaction starting solution should increase pore diameter by increasing the volume of the templating 
micelles while maintaining size and uniformity. As a result, the method's viability was assessed using decane (which had previously been used in the model paper) and DIPB (not initially used in the method).

The deposition of a silica layer is expected to trap larger molecules inside while allowing many small molecules (such as water, $0.28 \mathrm{~nm}$ ) to pass through. While typical Stöber silica synthesis products are regarded as non-porous, various studies have concluded that solid network silica chains have gaps that water molecules can traverse. ${ }^{63,65,66}$ A porosity level within the Stöber-type silicas is supported by evidence that gold particles in solid silica are leached away over time. ${ }^{67}$ The surfaces' hydrophilic structure allows water to flow into and out of the nanoparticle. The surface's porosity depends on specific synthesis parameters; ${ }^{63}$ controlling the thickness and porosity of the solid silica coating layer is possible.

This chapter describes a fairly reproducible literature method for making $80 \mathrm{~nm}$ std-MSNs In our adopted synthesis method; we used CTAB in place of CTAC because the former was readily available in the lab. Although the stirring rate was not specified quantitatively in the adopted method, we used a specified stirring speed of $1000 \mathrm{rpm}$ during the synthesis to improve reproducibility. Stirring rate has been identified as a critical player in nanoparticle size control, with higher stirring speeds producing smaller particles. ${ }^{71}$ The incorporation of decane and DIBP as swelling agents into the procedure demonstrates the potential of tailorability of MSNs in increasing pore diameters. However, there was a marked decrease in size and a partial loss in the sphericity of the MSNs. The necessity of developing reproducible methods for MSNs is a critical step in expanding MSNs 
applications beyond the bench.

\subsection{EXPERIMENTAL}

\subsubsection{Materials}

All reagents were purchased and used without further purification. Tetraethoxysilane (TEOS, 98\%) and ammonium fluoride $\left(\mathrm{NH}_{4} \mathrm{~F}, 98 \%\right)$ were purchased from Sigma-Aldrich. Cetyl trimethyl ammonium bromide (CTAB, 99\%) and absolute ethanol (EtOH, 200 proof) were purchased from Research Organics and Deacon labs, respectively. Triethylamine (TEA, 99\%)), decane (99\%) and di-isopropylbenzene (DIPB, 98\%) were purchased from Acros. Hydrochloric acid $(\mathrm{HCl})$ was purchased as an AR grade. Electrophoretically pure $\mathrm{H}_{2} \mathrm{O}\left(18 \mathrm{M} \Omega \bullet \mathrm{cm}\right.$ resistivity, called millipore $\mathrm{H}_{2} \mathrm{O}$ ) was used for all synthesis protocols. Laboratory deionized water $\left(\mathrm{DI}-\mathrm{H}_{2} \mathrm{O}\right)$ and ethanol (95\% Fisher) was used for all washing steps. All reactions, washings, and analyses were done in ambient laboratory conditions unless otherwise indicated. Experiments were repeated at least three times with similar results. For representative samples, the mean \pm standard deviation was reported.

\subsubsection{Synthesis of MSNs}

MSNs were synthesized using a modified literature Stöber method, similar to procedures used by Moller ${ }^{95}$ using CTAB as the surfactant instead of CTAC. The overall synthesis scheme is represented in Figure 2.3. and gives rise to the product we refer to as standard MSNs (std-MSNs). This standard method was modified to include decane and DIBP swelling agents, giving rise to MSNs with enlarged pore diameters; we refer to these 
products as dec-MSNs and DIPB-MSNs, respectively.

Two reagent mixtures were set up for the standard procedure: 1) the micelle colloid and 2) the silane mixture. $0.2 \mathrm{~g} \mathrm{NH}_{4} \mathrm{~F}$ was combined with $44 \mathrm{~mL}$ millipore $\mathrm{H}_{2} \mathrm{O}$ and $1.33 \mathrm{~g} \mathrm{CTAB}$ in a $250 \mathrm{~mL}$ round-bottomed flask stirred at $500 \mathrm{rpm}$ on a hot plate kept at $60{ }^{\circ} \mathrm{C}$ to form the micelle colloid. At least 25 minutes were spent stirring this mixture. The silane mixture was made by carefully adding $4 \mathrm{~mL}$ of TEOS to $25 \mathrm{~mL}$ of TEA in a $50 \mathrm{~mL}$ polypropylene centrifuge tube. The tube was capped and immersed in a water bath maintained at $90{ }^{\circ} \mathrm{C}$

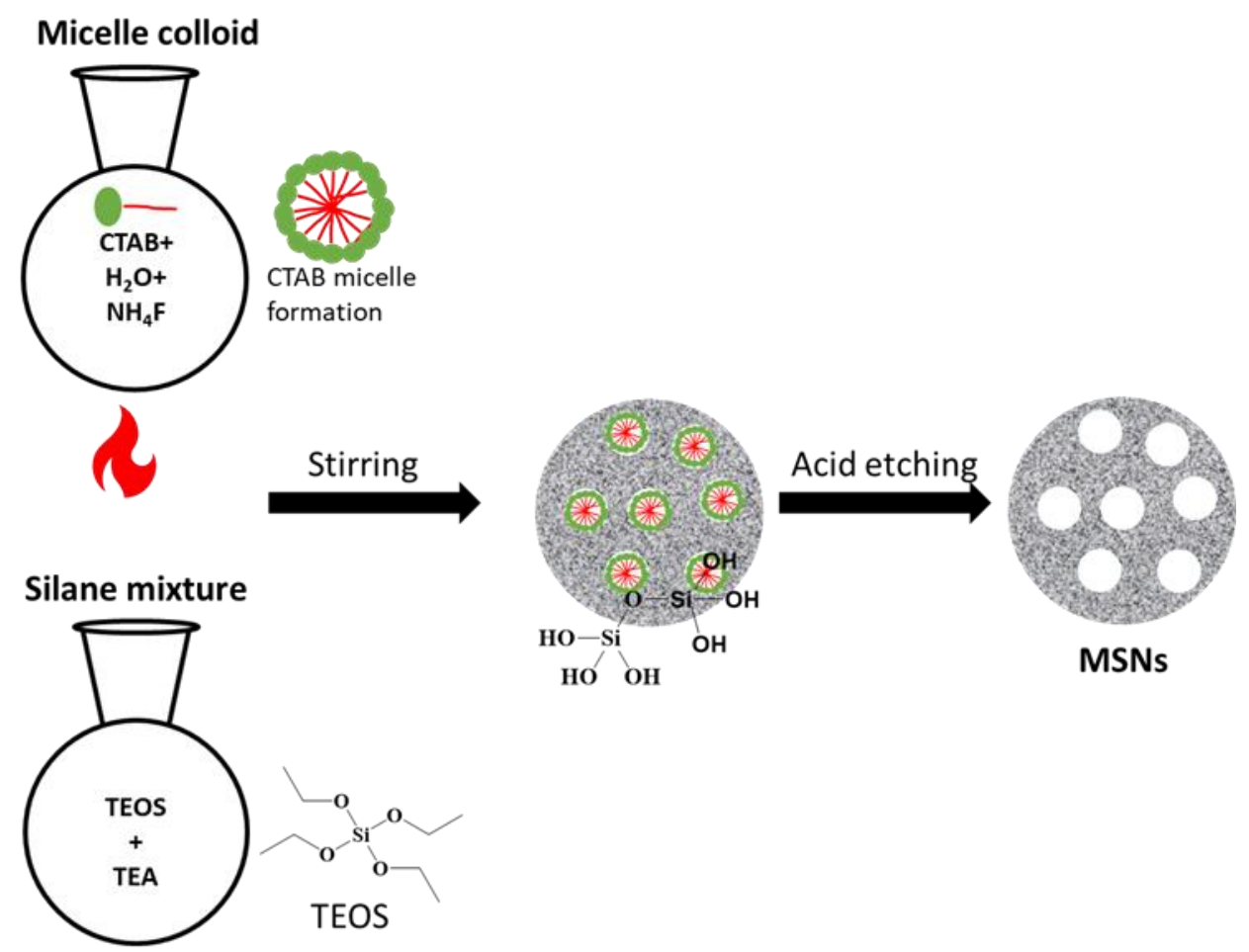

Figure 2.3 CTAB-based reaction scheme for the synthesis route of MSNs. Silicic acid monomers' condensation occurs on the CTAB micelle's surface following the mixing of the micelle colloid and silane mixture. Two partially condensed silicic acid monomers are depicted on the surface template-filled pore. Acid etching opens the pores, allowing MSNs to form.

for 30 minutes with the contents still unmixed. Following that, this mixture was added all 
at once to the stirring micelle colloid, followed by a rapid transfer of the entire mixture to a beaker fitted with a stir bar and spinning at $1000 \mathrm{rpm}$ on a room temperature stir plate; the reaction continued overnight.

Subsequently, the resulting suspension was centrifuged at $18000 \mathrm{Fc}$ and redispersed in a $70 \% \mathrm{v} / \mathrm{v} \mathrm{EtOH} / \mathrm{H}_{2} \mathrm{O}$ solution. The centrifugation-dispersion procedure was repeated with $95 \% \mathrm{v} / \mathrm{v} \mathrm{EtOH}$ to isolate a pellet of CTAB-templated MSNs after discarding the supernatant. To free the CTAB-filled pores, the pellet was resuspended in a $100 \mathrm{~mL}$ EtOH/HCl solution $(9: 1 \mathrm{v} / \mathrm{v})$ and refluxed at $90{ }^{\circ} \mathrm{C}$ for 1 hour. After cooling down the suspension to room temperature, the nanoparticles were centrifuged and went through four washing cycles before being analyzed by FT-IR. The etching procedure was repeated until no CTAB was detectable by FT-IR analysis. Finally, the CTAB-free MSNs were ovendried at $60^{\circ} \mathrm{C}$ overnight.

Decane and DIPB were incorporated into the micelle mixture of the standard method at a ratio of 1:1.3 (TEOS: swelling agent) to evaluate the effectiveness of swelling agents in increasing pore diameter. This resulted in the synthesis of dec-MSNs and DIPB-MSNs, respectively. After synthesis, the swelling agents and micelle templates were removed from these samples, as described for the std-MSNs above.

\subsubsection{Characterization of synthesized MSNs}

The morphologies of the synthesized nanoparticles were analyzed by TEM using a Technai F20 TEM operating at $4500 \mathrm{eV}$ (CEMN, Portland State University). Samples for analysis 
were thoroughly sonicated in EtOH, drop cast onto type-B carbon-coated copper TEM grids (Ted Pella product \#1844-F), and air-dried for about 10 minutes at room temperature before being fixed by heating at $100{ }^{\circ} \mathrm{C}$ for at least 30 minutes. The resulting TEM images were analyzed using the FIJI software package. The size distribution data (average and standard deviation) was determined by measuring at least 100 nanoparticles from each sample without selective measuring.

The composition and purity of the synthesized MSNs were investigated by FT-IR using a Thermo Nicolet iS10 spectrophotometer equipped with a single-bounce diamond attenuated total reflectance (ATR) attachment. A liberal amount of the dried $\left(\right.$ at $70{ }^{\circ} \mathrm{C}$ overnight) ground sample was placed directly onto the diamond crystal and gently pressed onto its surface using a spatula before measuring. For each sample, a total of 16 scans were collected from $4000-650 \mathrm{~cm}^{-1}$, and the signal was averaged to yield the final IR spectrum.

$\mathrm{N}_{2}$ porosimetry isotherms were obtained at $77 \mathrm{~K}$ using Micromeritics ASAP 2020 surface area analyzer. Specific surface areas were calculated using Brunauer-Emmett-Teller (BET), while the pore size was calculated using the Barrett, Joyner, and Halenda (BJH) adsorption curves. MSNs were dispersed in hexanes and dried at room temperature under vacuum for 18 hours. Before analysis, the samples were activated at $448 \mathrm{~K}$ for at least 24 hours to remove the solvent and trapped gas. Activation was considered complete when the outgassing rate fell below $2.5 \mu$ torr $\mathrm{min}^{-1}$. The sample mass was determined by the difference in mass between the empty sample tube and the loaded sample tube post- 
activation.

\subsection{RESULTS AND DISCUSSION}

\subsubsection{Synthesis of MSNs}

The modified Stöber method presented in Section 2.2.2 synthesized MSNs of varying NP diameters and pore sizes. The standard method was used to synthesis std-MSNs. The standard process was then modified to obtain MSNs with a larger pore diameter by incorporating decane and DIPB as swelling agents, resulting in dec-MSNs and DIPBMSNs, respectively. With stirring rate identified as a critical player in nanoparticle size control $^{22,71}$, we used a specified stirring speed of $1000 \mathrm{rpm}$ during the synthesis to improve reproducibility.

$\mathrm{CTAB}$, an amphiphilic surfactant, acts as the template and forms spherical micelles in the aqueous media. The outer hydrophilic surface of CTAB with the cationic head groups interacts with hydrolyzed, deprotonated silicate monomers (via electrostatic attractions). It creates small nucleation centers with the silicic acid/silicate monomer concentrated at the hydrophilic interface, resulting in an amorphous silica layer surrounding the micelles. Although $\mathrm{CTAB}$ is generally poorly soluble in water, the addition of ammonium ions to the reaction media is known to protonate the CTAB to a greater extent, resulting in increased solubility and enhanced control over nucleation rate. Moreover, ammonium has been shown to prevent particle aggregation ${ }^{96}$ in $\mathrm{SiO}_{2} \mathrm{NP}$ colloids by adjusting the ionic strength and helping to achieve electrostatic stabilization of the colloidal nanomaterials, 
which we also observed with $\mathrm{NH}_{4}{ }^{+}$addition.

In this standard method, $\mathrm{NaOH}$ base was replaced by TEA which served to control the hydrolysis and condensation rate of TEOS, allowing for colloidal solutions of MSNs with appropriate sizes and size distributions for further studies. The effectiveness of TEA in producing high-quality nanoparticles has been attributed to its ability to act as both a complexing agent for hydrolyzed silica species and an encapsulator for resultant MSNs. ${ }^{95}$ These features lead to growth limitation and aggregation inhibition making TEA-based synthesis procedures highly reliable and repeatable for making MSNs. Following synthesis, the surfactant was removed using $\mathrm{EtOH} / \mathrm{HCl}$ etching. The method relies on the acid's $\mathrm{H}^{+}$ions to interfere with the electrostatic attraction between the anionic silicate groups on the MSNs and the surfactant cationic head groups. The $\mathrm{H}^{+}$ions percolate the entire silica network and participate in an ion exchange with $\mathrm{CTA}^{+}$since the electrostatic interaction of the $\mathrm{CTA}^{+}$and the silicates at the interface are much weaker than the smaller $\mathrm{H}^{+}$ions. $^{97}$

\subsubsection{Characterization of MSNs}

FT-IR analysis was used to confirm specific silica functional groups and examine the etching protocol's effectiveness for removing CTAB molecules. To ensure that the pores were template-free, it was sometimes necessary to repeat the post-synthesis etching step until no CTAB peaks appeared. Figure 2.4 shows spectra of CTAB (A), unetched std- 


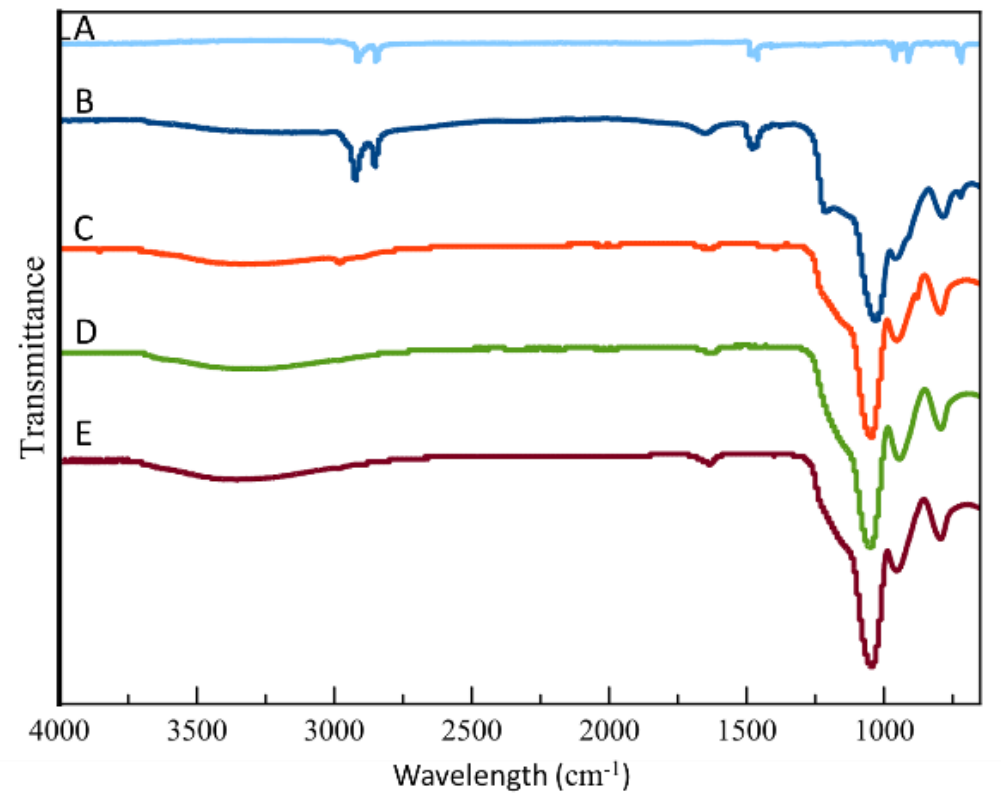

Figure 2.4 Infrared transmission spectra of A) CTAB, B) unetched MSNs, C) std-MSNs, D) dec-MSNs, and E) DIPB-MSNS

MSNs (B), and etched MSNs (C is std-MSNs, D is dec-MSNs, and E is DIPB MSNs, after removing the template). The CTAB spectrum (A) has peaks that occur at about $2920 \mathrm{~cm}^{-1}$ and $2850 \mathrm{~cm}^{-1}$, and these are caused by the asymmetric and symmetric vibrations of $-\mathrm{CH}_{2}$ groups. ${ }^{98,99}$ The two peaks are shown to be present in the unetched sample (B), and their presence in etched nanoparticles provided the basis for the process to be repeated until there were no CTAB peaks noted. The CTAB peaks are absent in the etched MSNs (C-E), indicating the absence of CTAB within the pores. The etched samples exhibit only the typical MSN peaks: $\mathrm{Si}-\mathrm{O}-\mathrm{Si}$ asymmetric stretching at about $1020 \mathrm{~cm}^{-1}, \mathrm{Si}-\mathrm{O}-\mathrm{H}$ asymmetric stretching around $960 \mathrm{~cm}^{-1}$, and Si-O-Si bending at $430 \mathrm{~cm}^{-1} \cdot 95,100$

The morphology, size distributions, and surface area of the particles were studied using 
TEM and $\mathrm{N}_{2}$ porosimetry studies. Figure 2.5 shows the TEM images, TEM size distributions, and the corresponding $\mathrm{S}_{\mathrm{BET}}$ and pore size measurements of representative images from triplicate experiments for std-MSNs, dec-MSNs, and DIPB-MSNs. Using our standard recipe with $\mathrm{CTAB}$ as the pore-generating template, we obtained nanoparticles $(78.6 \pm 8.2 \mathrm{~nm}$ ) with a wormhole arrangement (Figure 2.5 row 1), which is in close agreement with Bien et al. reported's measurements of 70-80 nm (the procedure we modified herein). 

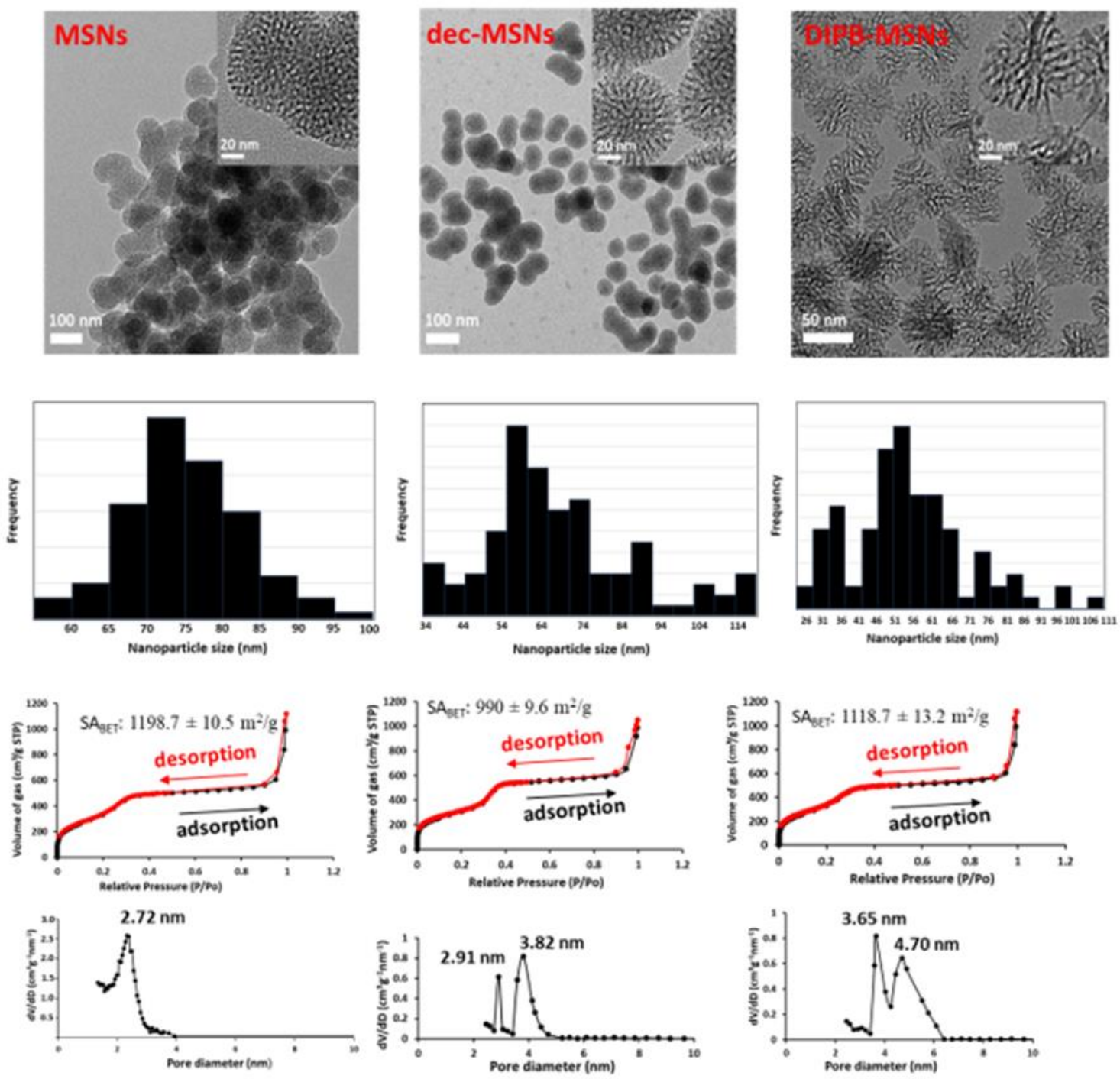

Figure 2.5 TEM images (Row 1), size TEM size distribution (Row 2), $N_{2}$ sorption isotherms (Row 3), and corresponding BJH pore size distributions curves of std-MSNs, dec-MSNs, and DIPB-MSNs

Although the size of the cationic surfactant head has been shown to affect nanoparticle size positively; for example, tosylate ion has increased size by at least $18 \%^{95,101}$; there appears to be no discernible difference when $\mathrm{Cl}^{-}$is replaced by $\mathrm{Br}^{-}$. This could be because the ionic difference between the two ions is insignificant (162 vs. $182 \mathrm{pm})$ enough to affect size noticeably. Our stirring speed could have also affected the size. To improve reproducibility, 
we intentionally used a stirring speed of $1000 \mathrm{rpm}$ during the synthesis. Stirring rate has been identified as a critical player in nanoparticle size control, with higher stirring speeds producing smaller NPs. ${ }^{22,71}$ If our chosen stirring rate was faster than the original method speed, the cation replacement effects could have been nullified by the faster stirring speed.

With the incorporation of decane and DIPB in the standard method, the pore morphology evolved from a wormhole structure to a more stellate architecture, as seen in the Figure 2.5 Row 1 (dec-MSNs and DIPB-MSNs). Contrary to what Bien et al. noted, the incorporation of swelling agents at a 1:1.3 ratio (TEOS to the swelling agent) resulted in a marked reduction in the size of the resultant nanoparticles; $61.2 \pm 17.3 \mathrm{~nm}$ and $49.2 \pm 19.3 \mathrm{~nm}$ for dec-MSNs and DIPB-MSNs respectively, as shown in the size distribution of representative of 100 nanoparticles from each type of MSNs (row 2). The size decrease could be because the incorporation of bulking agents lowers the $\mathrm{CMC}$, giving rise to multiple nucleation centers, resulting in size reduction. Other groups have noted this negative effect of swelling agents on the particles' size and monodispersity. ${ }^{102,103}$

Std-MSNs had the narrowest size distributions as indicated by the SD of 8.2. In contrast, dec-MSNs and DIPB-MSNs have 17.3 and 19.3, respectively, indicating a loss of uniformity with the introduction of swelling agents. Given the interconnection of nanoparticle synthesis factors, it is improbable that simply adding a bulking agent without optimizing starting concentration of the other reaction constituents will preserve the monodispersity and size. Careful optimization of reaction conditions might be required if uniform particles are to be realized. 
The size and distribution of the pores were determined using $\mathrm{N}_{2}$ porosimetry isotherms, and all three samples (std-MSNs, dec-MSNs, and DIPB-MSNs) showed the typical type IV isotherms (row 3) of predominantly mesoporous material. At low relative pressure, adsorption increased with rising relative pressure. At that time, the nitrogen molecules were adsorbed in single or multiple layers onto the inner surface of the sample's pores. When the relative pressure was increased gradually, an abrupt rise in adsorption occurred due to capillary condensation of $\mathrm{N}_{2}$ in the mesopores. After that, it became gradual until saturation was achieved. The determined $\mathrm{S}_{\mathrm{BET}}$ for std-MSNs, dec-MSNs, and DIPB-MSNs were $1198.7 \pm 10.5 \mathrm{~m}^{2} / \mathrm{g}, 990 \pm 9.6 \mathrm{~m}^{2} / \mathrm{g}$, and $1118.7 \pm 13.2 \mathrm{~m}^{2} / \mathrm{g}$ with no apparent trend noted The pore-size distributions were determined using the BJH method, and the obtained size distributions are shown in row 4. Std-MSN had narrow pore size distribution centered at around $2.7 \mathrm{~nm}$. Incorporating decane and DIPB into the synthesis procedure seems to have enlarged the pores (row 4). dec-MSNs size distribution had to peaks at $2.91 \mathrm{~nm}$ and 3.82 $\mathrm{nm}$ while DIPB-MSNs peaked at $3.65 \mathrm{~nm}$ and $4.7 \mathrm{~nm}$. We speculated that these hydrophobic molecules got trapped within $\mathrm{CTAB}$ micelles, bulking the template size, thus making the pore size bigger. The existence of two peaks within the size distributions of dec-MSNs and DIPB-MSNs indicated a lack of uniformity in the pore size distributions. This could have been caused by the inhomogeneous distribution of the swelling agent within the CTAB micelle. 


\subsection{CONCLUSIONS}

In this chapter, the synthesis and characterization of three types of MSNs were described. The method described here was adopted from literature, with the templating surfactant being CTAB instead of CTAC. This change resulted in nanoparticles (std-MSNs) with no discernible morphological and size differences to those reported in the literature source. We hypothesized that the ionic size difference between the $\mathrm{Cl}^{-}$and $\mathrm{Br}^{-}(162$ vs. $182 \mathrm{pm}$ ) could be insignificant. The swelling agents were incorporated to increase the pore size; decane has already been used in the synthesis, and DIPB has not been tried with this method. The incorporation of swelling-agent seems to have resulted in 1-size reduction, a deviation from the trends noted in the literature, 2-widening size distribution, and 3inhomogenous pore size distribution. Detailed explanations to the observations presented were given and justification supported through literature. We intentionally used a specific stirring speed as literature has identified it as a critical factor in batch-to-batch variations. The synthesis produced highly reproducible nanoparticles for std-MSNs with narrow size distributions.

Further improvement to size distribution for the swelling-agent modified methods should include optimizing the starting materials' molar ratios. Based on their size, uniformity, and desirable size, the std-MSN were then identified to progress to the next stage of functionalization, loading, and assessment presented in Chapter 4. 


\section{CHAPTER 3: SYNTHESIS OF HOLLOW MESOPOROUS SILICA NANOPARTICLES}

\subsection{INTRODUCTION}

hMSNs are advanced MSNs with an interior void space and homogenous pores integrated into a silica coating or shell, making them an excellent alternative for loading biomedical agents due to their superior loading capacity over conventional MSNs. The interior void size can be controlled by carefully selecting the void template, thus potentially providing a direct means of controlling the encapsulated molecular or ionic payload amount via synthesis. Further, we hypothesized that the thickness and porosity of the stable mesoporous $\mathrm{SiO}_{2}$ coating could provide a means of directly controlling the water exchange between the encapsulated and bulk water, making them an attractive, tunable host for CEST-MRI agent construction. The synthesis of hMSNs, including a facile delayedaddition Stöber synthesis procedure and characterization of the hMSNs, will be discussed in detail. The hMSNs synthesized in this Chapter will be used in our subsequent studies in Chapter 4 (functionalization, loading, and coating).

\subsubsection{Synthesis of hMSNs}

hMSNs have been fabricated using three main methods: device-based synthesis, in situ template synthesis, and sacrificial template synthesis. ${ }^{104-106}$ The device-based methods use some equipment, like spray pyrolysis or nozzle processes, to synthesize hMSNs. For example, hMSNs were synthesized from preformed small silica suspensions deposited onto emulsion droplet templates using the spray drying approach. In the spray drying approach, 
the solvent is rapidly evaporated, which causes silica particle clusters to form on the interface of the templates. ${ }^{107-110}$ Because this procedure is a continuous process, it offers the possibility of scalable production of hMSNs. The resulting hMSNs, on the other hand, are prone to aggregation. Due to the random assembly of the sprayed colloids at the emulsion droplet surfaces, the resultant particles lack a continuous amorphous silica shell and exhibit a significant degree of observable polydispersity. Finally, the nanoparticles generated using this approach are typically micron-sized, limiting their biomedical application potential. ${ }^{106,111}$

The in-situ template method allows the fabrication of hollow nanoparticles without additional reagents by using intermediates or by-products as templates, eliminating the need for etching or calcination as the template is automatically removed at the end of the reaction. Wang et al. created hollow hMSNs using styrene droplets as a soft template and in situ polymerized polystyrene/silica nano-domains as a hard template. Because of its amphiphilicity, the methyltriethoxysilane pre-hydrolysate attached to the styrene droplets and then proceeded hydrolysis-condensation to form the mesoporous silica shell. Then, using a mesoporous silica shell as a nanoreactor, a portion of the in situ polymerized polystyrene/silica chains migrated to the outer surface of the mesoporous silica shell due to strong capillary force in the mesoporous channels. At the same time, some siloxane oligomers migrated due to apparent interfacial activity, resulting in hierarchical hMSNs. ${ }^{112}$

The in-situ method has also been extended to include the use of void structures as templates. The introduction of bubbles into the starting reactant solution introduces 
chemical heterogeneity into the synthesis solution at the solvent-template interface that lowers the surface energy and is vital for promoting crystal assemblies. ${ }^{104}$ The in situ method is advantageous in offering a low-cost, high-productivity method. Furthermore, the lack of a sacrificial template makes it less time-consuming due to fewer required synthetic steps. However, size uniformity is difficult to control using the in-situ synthesis method.

The sacrificial template methods remain the most commonly used approach for synthesizing hMSNs. These methods involve a minimum of two main synthetic steps: First, a silica coating is deposited onto an already formed core template. Then, selective dissolution of the core template gives rise to a hollow cavity inside the remaining $\mathrm{SiO}_{2}$ shell. The used etchant (whether acidic/basic) depends on the physicochemical nature of the template (e.g., organic compound, elemental metal, inorganic compound). These methods have traditionally utilized soft and hard templates. Hard templates have gradually gained popularity despite their time-consuming synthesis processes, most likely because it is relatively easier to control the morphological and size $(30-100 \mathrm{~nm})$ characteristics of the resulting hMSNs when using more rigid templates. ${ }^{106,111}$ Table 3.1 summarizes the different templates that have been used in synthesizing hMSNs and compares their advantages and disadvantages.

Lastly, the pore-forming surfactants in the shell are removed by either calcination or etching to provide empty pores in the remaining silica shell. Selective dissolution (explained in detail in Section 3.1.2) is generally preferred over calcination because treating the nanoparticle at high temperatures can cause irreversible aggregation due to 
dehydration and crosslinking between particles. Moreover, the high-temperature treatments may drastically reduce the number of functional surfaces silanol groups needed for further functionalization. ${ }^{92,126}$

Table 3. Advantages and disadvantages of templating strategies used in hMSNs synthesis

\begin{tabular}{|c|c|c|c|c|}
\hline \multicolumn{2}{|l|}{ Template type } & Advantages & Disadvantages & \multirow{2}{*}{$\begin{array}{l}\text { References } \\
106,113-115\end{array}$} \\
\hline Soft templates & $\begin{array}{lr}\text { Polymer } & \text { aggregates } \\
(e . g ., \text { PVP and PTMS) } \\
\text { surfactant micelle }(e . g . \\
\text { CTAB), } \\
\text { droplets }\end{array}$ & $\begin{array}{l}\text { Well established } \\
\text { synthesis process }\end{array}$ & $\begin{array}{l}\text { Limited control of } \\
\text { size, typically } \\
\text { produces very small } \\
\text { hMSNs, low } \\
\text { synthesis yield }\end{array}$ & \\
\hline \multirow[t]{4}{*}{$\begin{array}{l}\text { Hard } \\
\text { templates }\end{array}$} & Bacteria, yeast cells & $\begin{array}{l}\text { Different shapes of } \\
\text { hMSN }\end{array}$ & $\begin{array}{l}\text { High cost, not } \\
\text { scalable, difficulty } \\
\text { in size control }\end{array}$ & 116,117 \\
\hline & $\begin{array}{l}\text { Polymer templates (e.g., } \\
\text { polystyrene and PAA }\end{array}$ & $\begin{array}{l}\text { Precise size control, } \\
\text { well-established } \\
\text { synthesis process }\end{array}$ & Low yield, high cost & $\begin{array}{l}118-120 \\
\end{array}$ \\
\hline & $\begin{array}{l}\text { Inorganic particles (e.g., } \\
\text { carbon, Zinc oxide, } \\
\text { calcium carbonate) }\end{array}$ & Precise size control & $\begin{array}{l}\text { Time-consuming, } \\
\text { high cost, low yield }\end{array}$ & $121-123$ \\
\hline & Solid silica & $\begin{array}{l}\text { Precise size control, } \\
\text { well-known } \\
\text { chemistry, } \\
\text { Inexpensive }\end{array}$ & Time-consuming & $111,124,125$ \\
\hline
\end{tabular}

PVP: Polyvinylpyrrolidone; PTMS: Phenyltrimethoxysilane

\subsubsection{Mechanisms of templated-hMSNs synthesis}

The synthesis of hMSNs usually follows a dual-templating procedure: one template creates the central hollow void, and another template creates the mesopores in the silica shell, as described above. The mesoporous silica coating is usually deposited onto a spherical sacrificial template using the standard Stöber method. Figure 3.1 illustrates the process. The template is introduced to a silica precursor solution containing pore-forming compounds (surfactants). For shell deposition to be successful, the template surface must 
carry functional groups that support silica deposition, or else an additional surface activation step will be required. Just as in MSN synthesis, the precursor is hydrolyzed under basic conditions to form oligomers that electrostatically interact with surfactant micelles. Because of the presence of the template, instead of creating new nucleation centers, the silica deposition takes place on the surface of the template, giving rise to template-mesoporous silica hybrid nanoparticles. ${ }^{77,91}$

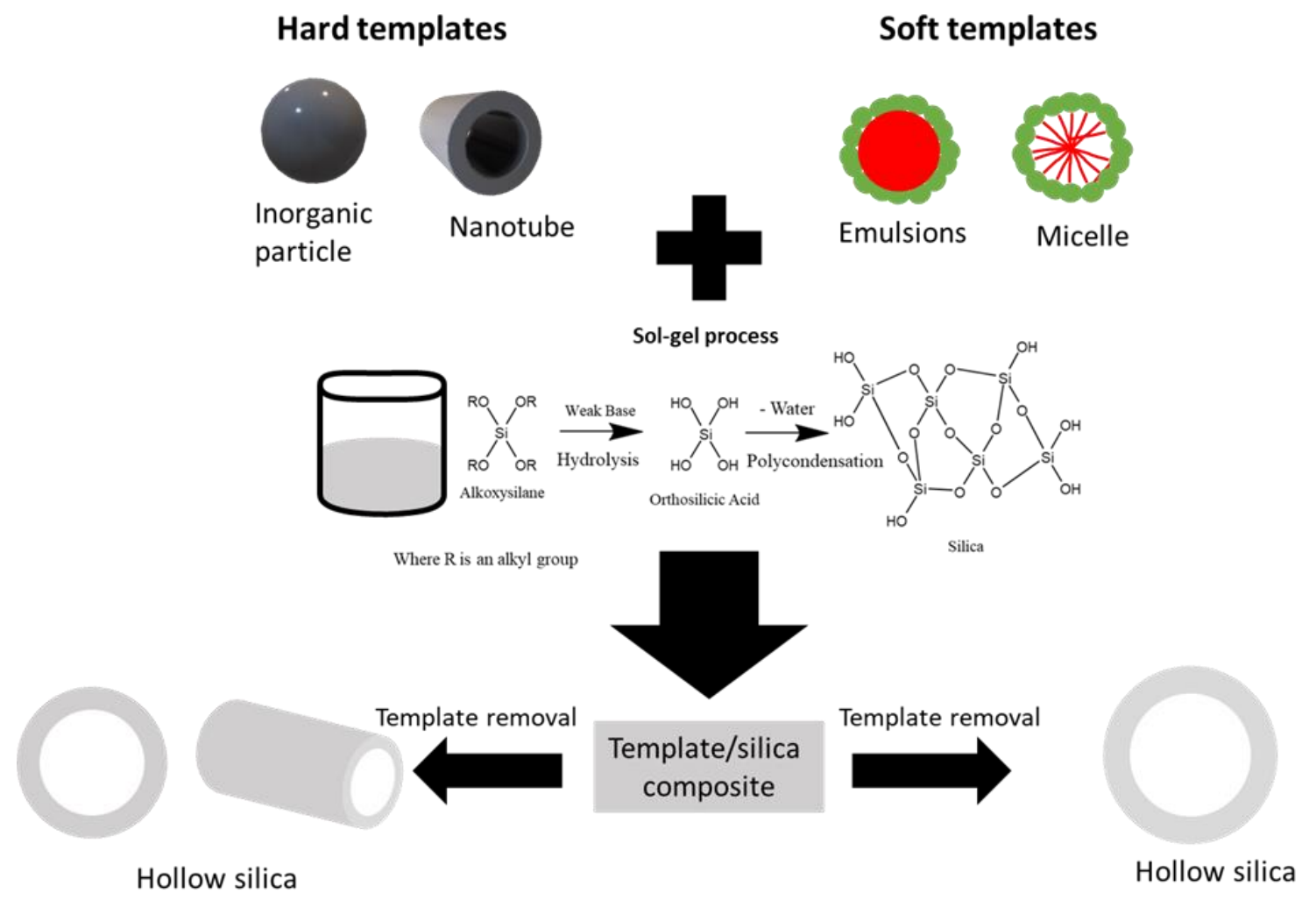

Figure 3.1 Schematic illustration for the template synthesis of hollow $\mathrm{SiO}_{2} \mathrm{NPs}$ (Adapted from Bao et al., $2016)^{106}$

Depending on the nature of the template, it is usually followed by selective removal of the template. Acidic or basic etching treatments that use nitrates or acid to oxidize the core 
material apply to organic matter and inorganic (especially metal oxides) templates. Polymer templates such as polystyrene or polymethylmethacrylate (PMMA) can also be etched selectively with organic solvents such as toluene. ${ }^{127,128}$ Calcination is another popular method for removing polymer templates. This procedure entails heating dried mesoporous silica samples for at least five hours in an oven set at or above $500{ }^{\circ} \mathrm{C}$. The surfactants that reside within the pores decompose at high temperatures, leaving the desired mesoporous silica framework intact.

\subsubsection{Motivation and Scope}

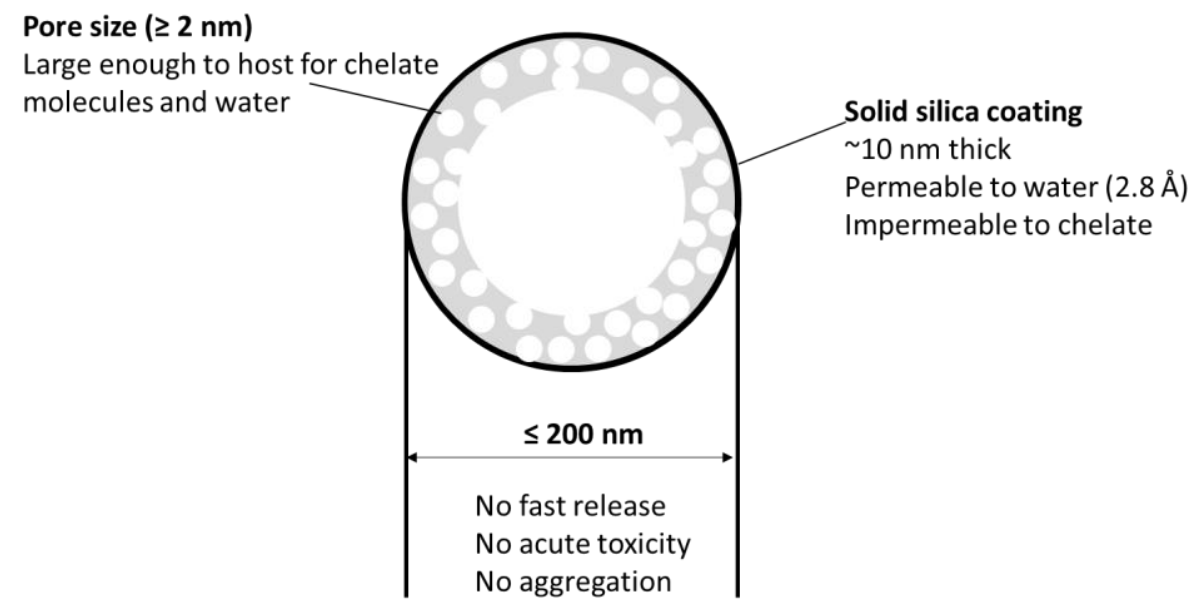

Figure 3.2 Ideal hMSN-based host demonstrating appropriate target parameters for CEST-MRI application hMSNs have an internal void that can operate as a reservoir for small molecule contrast agents, in addition to possessing the good physical and chemical qualities of MSNs (adjustable pore size, chemical and thermal stability, large surface area, superior biodegradability, and biocompatibility). The large interior volume has rendered them superior loading capacity than their MSNs counterparts without the hollow interior. We 
envisioned synthesizing hMSNs with control over the void size and shell thickness would allow us to independently tailor the two important structural parameters for optimum CEST-MR contrast generation. The large void can encapsulate a large payload and pool of water for exchanging protons, and the shell thickness and porosity could permit fine-tuning of the water exchange rate. By changing the core sizes in these nanoparticles, we can control the number of water molecules encapsulated. These features would mean hMSNs represent an ideal candidate for CEST-MRI agents. Although MSNs have been explored, hMSNs are yet to be explored in this application, and an hMSN host designed to have the feature illustrated in Figure 3.3 below will be an ideal candidate.

Nanoparticle diameters of $100-200 \mathrm{~nm}$ are considered optimum for avoiding rapid release, acute toxic impact, and aggregation in physiological fluids, blood capillaries, and alveoli. Our decision to adopt hMSNs as a host was motivated by hMSNs' relative volume (hollow interior) to liposomes, the leading nano-based CEST agent explored so arr $^{46}$ Zhao et al. discovered that CEST efficiency declined as liposome size increased due to a decrease in the surface-to-volume ratio, hinting that nanoparticles smaller than $90 \mathrm{~nm}$ may have a greater effect. However, they discovered that the best sizes for biodistribution and lipoCEST contrast are in the range of $90-200 \mathrm{~nm},{ }^{42}$ which corresponds to our synthesis targets. A starting size of around $150 \mathrm{~nm}$ was chosen to allow a thin silica shell $(10 \mathrm{~nm})$ deposition that successfully traps the chelate. Pore diameters greater than $2 \mathrm{~nm}$ should be large enough to allow guest molecules and water into the inside cavity. As mentioned in Chapter 2, a thin silica coating should allow for water $(2.8 \AA)$ circulation between the 
interior and outside of the NP while successfully trapping the big guest molecules.

This Chapter describes a modified literature-based procedure for making hMSNs using solid $\mathrm{SiO}_{2}$ as core templates. We used one of the synthesized solid $\mathrm{SiO}_{2}$ to synthesize hMSNs by first depositing a mesoporous silica shell. Afterward, we performed a structuralselective etching step to remove the core template following the literature procedure with slight modifications. To tackle the problem of reproducibility associated with dropwise addition methods, we developed a delayed-addition method which, compared to an all-atonce addition method (across 3 trials), showed some noticeable improvement in the monodispersity of solid $\mathrm{SiO}_{2}$ NPs. We consistently made 3 different sizes of the solid $\mathrm{SiO}_{2}$ NPs by changing the catalyst concentration, an important experimental outcome in tailoring different sized hMSNs.

\subsection{EXPERIMENTAL}

\subsubsection{Materials}

Tetraethoxysilane 98\% (TEOS), aminopropyltriethoxysilane $\geq 98.0 \%$ (APTES), triethylamine $\geq 99.0 \%$ (TEA), anhydrous sodium carbonate, granular, $\geq 99.5 \%\left(\mathrm{Na}_{2} \mathrm{CO}_{3}\right)$, ACS reagent grade hydrochloric acid, $37 \%$ w/w aqueous solution $(\mathrm{HCl})$ and cetyltrimethylammonium bromide $(\mathrm{CTAB}, \geq 99.0 \%)$ were purchased from Sigma-Aldrich. Ammonium hydroxide as a $30 \% \mathrm{w} / \mathrm{w}$ aqueous solution $\left(\mathrm{NH}_{4} \mathrm{OH}(\mathrm{aq})\right)$ was purchased from Fisher. Absolute EtOH 200 proof (absolute EtOH) was bought from Decon labs. All synthesis reagents were utilized without additional purification, and all synthesis 
procedures used electrophoretically purified $\mathrm{H}_{2} \mathrm{O}$ with a resistivity of $18 \mathrm{M} \Omega \bullet \mathrm{cm}$ (Millipore, mp $\left.\mathrm{H}_{2} \mathrm{O}\right)$. Laboratory deionized water $\left(\mathrm{DI}-\mathrm{H}_{2} \mathrm{O}\right)$ and ethanol $(\mathrm{EtOH}$, reagent grade) was used for all washing steps. All reactions, washings, and analyses were done at ambient laboratory conditions unless otherwise stated.

\subsubsection{Synthesis of hMSNs}

hMSNs were synthesized according to a literature method, ${ }^{129}$ where the $\mathrm{SiO}_{2}$ core template was first produced using a facile delayed-addition Stöber synthesis procedure. The synthesis of hMSNs comprises four steps: 1. synthesis of solid $\mathrm{SiO}_{2}$ template (synthesized using the delayed-addition method), 2. deposition of a mesoporous silica shell on the template, 3. selective dissolution etching to remove the core template, and 4. shell etching to remove the CTAB from the mesopores in the silica shell.

\subsubsection{Synthesis of solid $\mathrm{SiO}_{2}$ template}

Two synthesis methods were explored to synthesize the solid $\mathrm{SiO}_{2}$ core template; 1) a delayed-addition method in which the TEOS is allowed to mix with the solvent before adding the catalyst and hydrolyzing agent (Procedure A), and 2) an all-at-once method in which all reagents are added together (Procedure B).

\section{Procedure A}

In this delayed-addition method, $80 \mathrm{~mL}$ of absolute EtOH and $4 \mathrm{~mL}$ of TEOS were added in a $100 \mathrm{~mL}$ round-bottomed flask and stirred at $450 \mathrm{rpm}$ at room temperature for $30 \mathrm{mins}$. After that, a pre-mixed solution of $\mathrm{mp} \mathrm{H}_{2} \mathrm{O}(1.29 \mathrm{~mL})$ and aqueous $\mathrm{NH}_{4} \mathrm{OH}(4 \mathrm{~mL})$ was 
added to the ethanolic TEOS solution. The $\mathrm{NH}_{4} \mathrm{OH}$ amount was then increased to synthesize different-sized nanoparticles. The absolute EtOH volume was subsequently adjusted to ensure a constant volume so that the other reagents remained at a constant concentration. The reaction mixture was left to stir at room temperature overnight. The resulting colloidal $\mathrm{SiO}_{2} \mathrm{NPs}$ were centrifuged at 18,000 Fc for 20 minutes and re-dispersed in $70 \% \mathrm{v} / \mathrm{v}$ aqueous EtOH by sonicating. The centrifugation-dispersion procedure was repeated three more times: first time with $70 \% \mathrm{v} / \mathrm{v}$ aq. $\mathrm{EtOH}$ and twice with $95 \% \mathrm{v} / \mathrm{v}$ aq.

EtOH. Following the final wash, the nanoparticles were re-dispersed in $17.5 \mathrm{~mL}$ absolute $\mathrm{EtOH}$ (giving a final concentration of $51.4 \mathrm{mg} / \mathrm{ml}$ ) for storage, subsequent use, and analysis.

Procedure B

In this all-at-once method (a comparative method to Procedure A), $80 \mathrm{~mL}$ of absolute EtOH, $4 \mathrm{~mL}$ of TEOS, $1.29 \mathrm{~mL} \mathrm{mp} \mathrm{H}_{2} \mathrm{O}$, and $4 \mathrm{~mL}$ aqueous $\mathrm{NH}_{4} \mathrm{OH}$ were stirred in a $100 \mathrm{~mL}$ round-bottomed flask at $450 \mathrm{rpm}$. The reaction mixture was left to stir at room temperature overnight, and subsequent washing procedures were done as in Procedure A.

\subsubsection{Deposition of a mesoporous silica shell}

3.5 $\mathrm{mL}$ aliquot of the stock suspension of solid $\mathrm{SiO}_{2}$ NPs from Section 3.2.2.1 was put into a $100 \mathrm{~mL}$ round-bottom flask to perform the next step of depositing a mesoporous shell onto the $\mathrm{SiO}_{2}$ core templates. $0.06 \mathrm{~g}$ of $\mathrm{CTAB}, 33 \mathrm{~mL}$ of water, and $28 \mu \mathrm{L}$ of TEA were added to the suspension, followed by stirring for 50 minutes at $80{ }^{\circ} \mathrm{C}$ at $600 \mathrm{rpm}$. The 
stirring speed was increased to $1400 \mathrm{rpm}$ after that, and $144 \mu \mathrm{L}$ of TEOS was added. The reaction was stirred for a further 4 hours. The mesoporous silica-coated $\mathrm{SiO}_{2}$ core templates were centrifuged at $18,000 \mathrm{Fc}$ for 20 minutes before rinsing three times with $95 \% \mathrm{v} / \mathrm{v}$ aq. $\mathrm{EtOH}, 70 \%$ v/v EtOH, and DI water, respectively.

\subsubsection{Core etching}

To create the hollow central void, mesoporous silica-coated solid $\mathrm{SiO}_{2}$ nanoparticles were re-suspended in $10 \mathrm{~mL} \mathrm{mp} \mathrm{H}_{2} \mathrm{O}$ and sonicated until homogeneous. Meanwhile, $1.33 \mathrm{~g}$ of sodium carbonate was mixed with $10 \mathrm{~mL} \mathrm{mp} \mathrm{H}_{2} \mathrm{O}$ at a stirring rate of $600 \mathrm{rpm}$ for 30 minutes at $50{ }^{\circ} \mathrm{C}$. The sonicated suspension was then added to the sodium carbonate solution, and the stirring rate was increased to $1200 \mathrm{rpm}$. The etching reaction proceeded for 10 hours under these conditions, and afterward, the resultant etched particles were washed three times with DI water, 70\% v/v aq. EtOH, and 95\% v/v aq. EtOH, respectively.

\subsubsection{Shell etching}

To remove the CTAB surfactant from the pores of the mesoporous silica shell, the resultant pellet from the final centrifugation in Section 3.2.2.3 was resuspended in $100 \mathrm{~mL}$ of an EtOH/ $\mathrm{HCl}$ solution $(9: 1 \mathrm{v} / \mathrm{v})$ and refluxed at $90{ }^{\circ} \mathrm{C}$ for 1 hour. Afterward, the nanoparticles were washed three times with $95 \%$ v/v aq. $\mathrm{EtOH}, 70 \%$ v/v aq. $\mathrm{EtOH}$, and lastly DI water, respectively. A sample of the acid-etched and washed nanoparticles was analyzed using FTIR spectroscopic analysis as described in the next session. The above etching procedure was repeated until no CTAB was detectable by FT-IR spectroscopic analysis. The CTAB- 
free hMSNs were then oven-dried at $60{ }^{\circ} \mathrm{C}$ overnight.

\subsubsection{Characterization}

FT-IR spectroscopy was used to analyze the microstructural composition of the hMSNs. FT-IR measurements were made on a Thermo Nicolet iS10 spectrophotometer with a single-bounce diamond attenuated total reflectance (ATR) attachment. For each measurement, a liberal amount of the dried, solid sample was placed directly onto the diamond crystal and gently pressed down using a spatula before measuring. The final IR spectra were calculated by averaging 16 scans from $4000-650 \mathrm{~cm}^{-1}$ for each sample.

The sizes and morphologies of the nanoparticles at various stages in the synthesis procedure were analyzed by TEM using a Technai F20 TEM operating at $4500 \mathrm{eV}$ (CEMN, Portland State University). To prepare samples for TEM analysis, NPs were thoroughly sonicated in EtOH and drop cast onto type-B carbon-coated copper TEM grids (Ted Pella product \#1844-F). The sample grids were air-dried for about 30 minutes at room temperature before being fixed by drying at $100{ }^{\circ} \mathrm{C}$ for another 30 minutes. The resulting TEM images were analyzed with the FIJI software package. The size distribution data reported herein were obtained by measuring and averaging the diameters of at least 100 nanoparticles, with all nanoparticles in the field of view counted to reduce bias in averaging the sizes.

The hydrodynamic radius of the NP samples in their re-dispersed aqueous colloidal form $(0.43 \mathrm{mg} / \mathrm{mL})$ was measured using a Horiba LB-550 DLS particle analyzer. $\mathrm{N}_{2}$ porosimetry 
isotherms were obtained at $77 \mathrm{~K}$ using a Micromeritics ASAP 2020 surface area analyzer. As indicated in Chapter 2, the surface areas of the samples were calculated using BET ${ }^{130}$ theory using isotherm adsorption. The JBH method was used to determine the pore size distributions using the adsorption isotherm. ${ }^{131}$ Before measurements, the NP samples were dispersed in hexanes and dried under vacuum for 18 hours at room temperature. Then, after placing them on the instrument, each sample was first thoroughly evacuated to remove adsorbed solvent and trapped gas by pulling vacuum at $\mathrm{T}=448 \mathrm{~K}$ for at least 24 hours. The evacuation was considered complete when the outgassing rate fell below $2.5 \mu$ torr $/ \mathrm{min}$. The sample mass was determined by the difference in mass between the empty sample tube and the loaded sample tube post-activation.

\subsection{RESULTS AND DISCUSSION}

\subsubsection{Synthesis of hMSNs}

The synthesis scheme used for the fabrication of hMSNs is shown in Figure 3.3. It comprises 4 main steps: 1) synthesis of solid $\mathrm{SiO}_{2} \mathrm{NP}$ using the Stöber method, 2) deposition of a mesoporous silica shell on the template using a modified Stöber method that included a surfactant to introduce porosity, 3) selective structural dissolution of the core using $\mathrm{Na}_{2} \mathrm{CO}_{3}$, and lastly, 4) $\mathrm{EtOH} / \mathrm{HCl}$ etching of the shell to remove CTAB from the mesopores. We followed the hMSN synthesis from the literature paper ${ }^{129}$ with slight modifications but used a delayed-addition procedure for synthesizing templating solid $\mathrm{SiO}_{2}$. The developed synthesis procedure produced uniformly sized nanoparticles in comparison to the all-at-once method $(92.1 \pm 10.0 \mathrm{~nm} v s 93.2 \pm 23.5 \mathrm{~nm}$, respectively) 


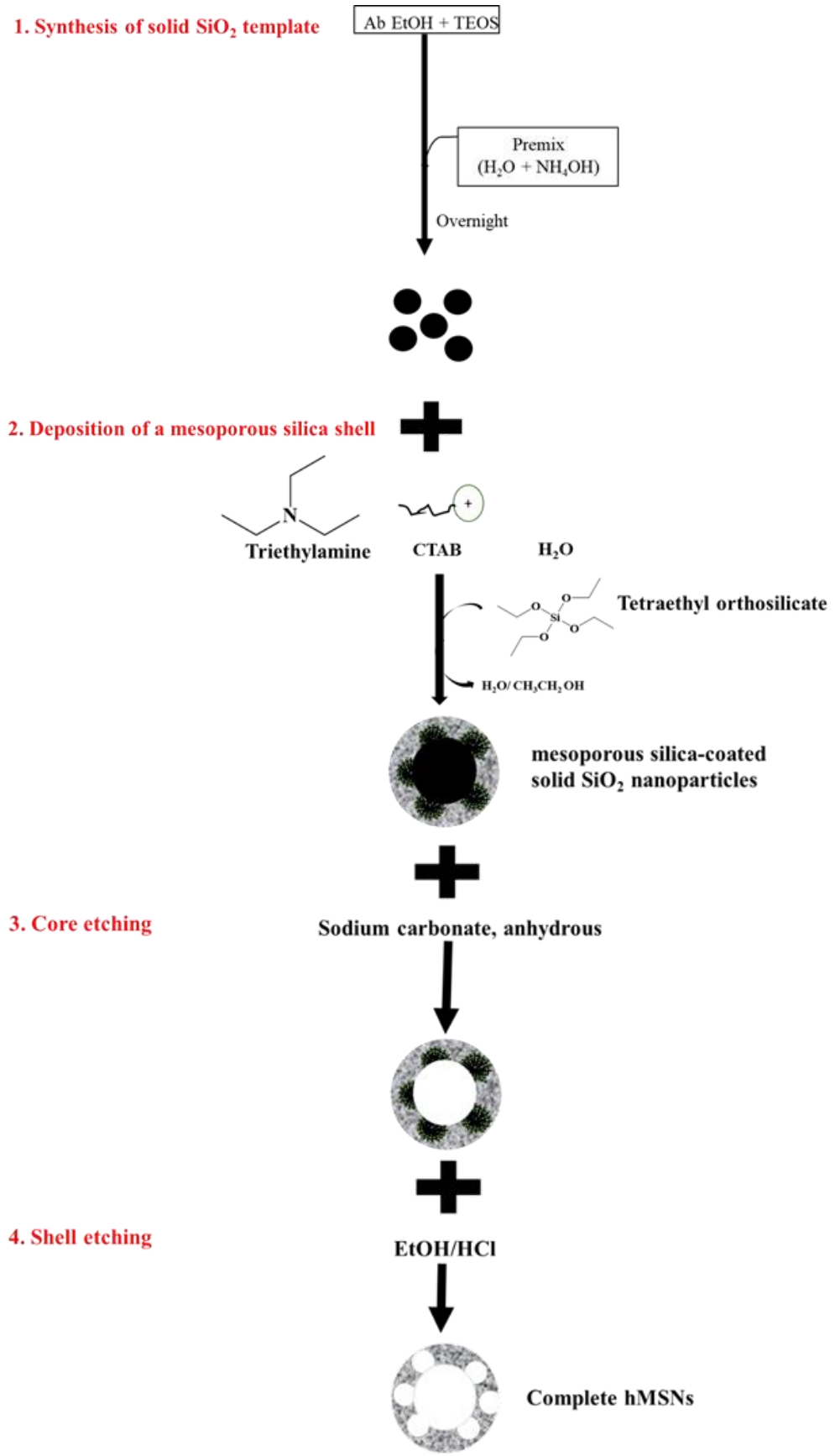

Figure 3.3 Synthesis scheme for the hMSNs 


\subsubsection{Synthesis of Stöber template}

The synthesis of hMSNs started with solid $\mathrm{SiO} 2 \mathrm{NP}$ synthesis, onto which a mesoporous silica layer was next deposited. The spheres' characteristics in size and shape are expected to translate to the final product, so the cores should be as uniform as possible. The original method used a dropwise method which resulted in very uniform nanoparticles (PD1 $<0.2$ ). After size differences were noted from batch to batch (ranging from $\sim 120-215 \mathrm{~nm}$ over 3 batches performed by 3 individuals), a thorough analysis of the method was thought to result from differences in TEOS addition rate during the TEOS-dropwise addition step; an observation supported by the literature. One study revealed that the particle size decreases as the rate of addition of TEOS increases regardless of temperature. ${ }^{132}$ Another study also reported that the feed rate impacts size though with contradictory trends. ${ }^{133}$ One way of addressing the challenge would have been using an all-at-once procedure, but though highly reproducible, such a method would have increased the size distribution. Using the same starting concentration and conditions from the paper, we thus developed a delayedaddition procedure to narrow the size distribution and increase batch-to-batch consistency (Procedure A). Procedure B is the comparable version of the method in which all the reactants were added at once. Figure 3.4 shows the schematic representation of these two procedures pursued under otherwise identical reaction conditions producing solid $\mathrm{SiO}_{2}$ templates with approximately the same size (representative batch of the 3 trials done). The TEM averages of the solid $\mathrm{SiO}_{2}$ NPs synthesized particles by Procedures $\mathrm{A}$ and $\mathrm{B}$ were $92.1 \pm 10.0 \mathrm{~nm}$ and $93.2 \pm 23.5 \mathrm{~nm}$, respectively. Thus, we find that Procedure A produced 

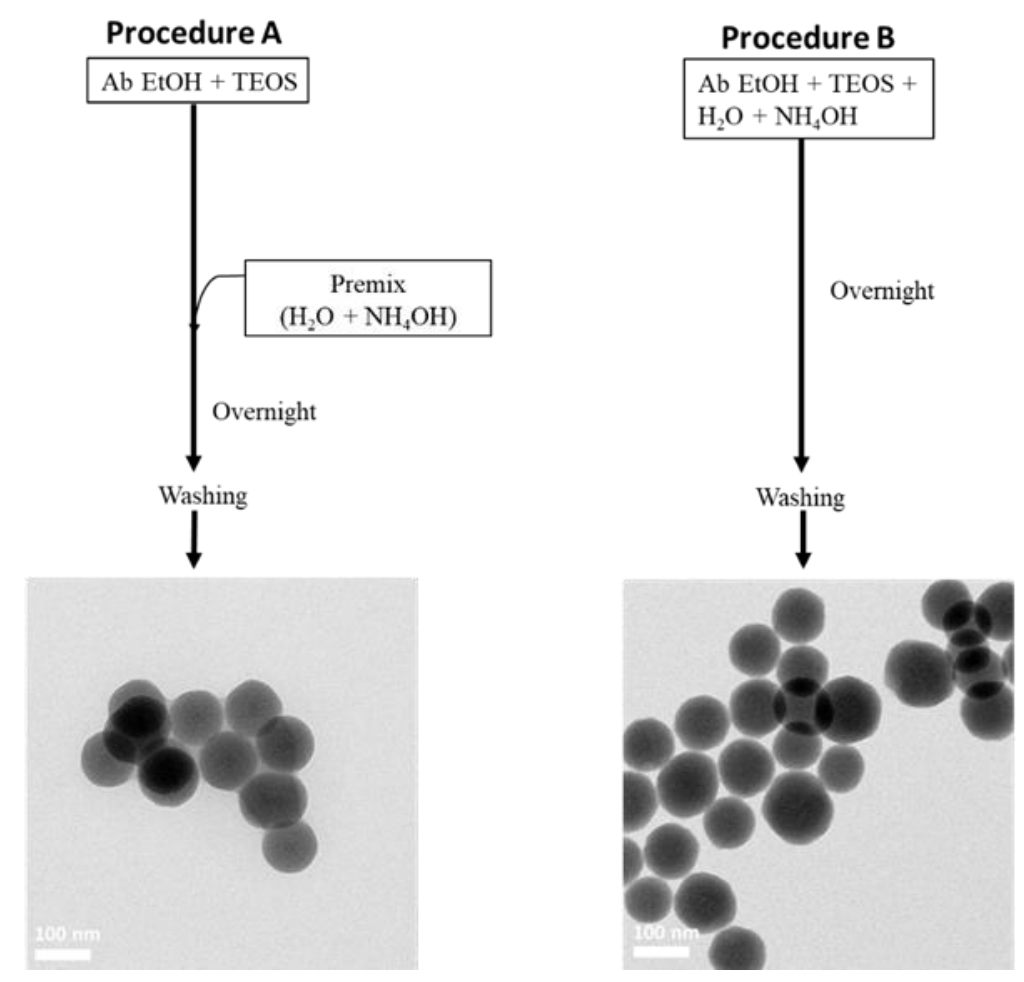

Figure 3.4 Schematic representation of the two different addition procedures (or reagent mixing procedures) explored in the synthesis of the solid $\mathrm{SiO}_{2}$ templates. Procedure A resulted in particles that were $92.1 \pm 10.0$ $\mathrm{nm}$ in comparison to Procedure B's $93.2 \pm 23.5 \mathrm{~nm}$

slightly better results in terms of narrowing the size distribution. These findings are consistent with prior research that showed that the order and rate of reagent addition provided a means to control particle size and that delayed mixing of the reagents can improve size homogeneity under otherwise consistent conditions (i.e., fixed concentrations of starting reactants and circumstances). ${ }^{133}$

The slight difference in uniformity between the two procedures is likely caused by TEOS interaction with other reactants, directly affecting TEOS homogeneity within the starting solution, although the actual mechanism is unclear. Water has also been observed to form several hydrogen bonding structure arrangements that have diverse shapes (e.g., dimer, 
trimer (cyclic), tetramer (cyclic)). ${ }^{134}$ These structures have various numbers of water molecules $\left(\mathrm{H}_{2} \mathrm{On}, \mathrm{n}=3-60\right)$. The presence of such structures is more likely in Procedure B and might contribute to lower homogeneity of TEOS molecules in the starting mixture leading to nanoparticles with broader size distributions. In Procedure A, mixing TEOS and EtOH before introducing water, in theory, allows for a greater TEOS homogeneity than in Procedure B. Further, the EtOH solvent effectively dilutes the TEOS, aiding the establishment of effective controlled interactions between the TEOS and water.

To change the solid $\mathrm{SiO}_{2}$ template size, the concentration of $\mathrm{NH}_{4} \mathrm{OH}$ used in the standard method was adjusted. EtOH as the primary solvent was used to even out the total volume of the starting solution to maintain the concentration of the other starting materials. Table 3.1 shows the starting material concentration and TEM with A-D corresponding to starting $\mathrm{NH}_{4} \mathrm{OH}$ concentrations of $0.56 \mathrm{M}, 074 \mathrm{M}, 0.91 \mathrm{M}$, and $2.22 \mathrm{M}$, respectively. Figure 3.5 shows the representative TEM images and DLS size distributions synthesized solid $\mathrm{SiO}_{2}$ NPs.

Table 3.1 Starting material concentration for different sized nanoparticles

\begin{tabular}{cccccc} 
Sample & $\begin{array}{c}\text { Absolute EtOH } \\
(\mathbf{M})\end{array}$ & $\begin{array}{c}\text { TEOS } \\
(\mathbf{M})\end{array}$ & $\begin{array}{c}\text { DI Water } \\
(\mathbf{M})\end{array}$ & $\begin{array}{c}\mathrm{NH}_{4} \mathrm{OH} \\
(\mathbf{M})\end{array}$ & $\begin{array}{c}\text { TEM Size } \\
(\mathbf{M e a n} \pm \mathbf{S D} \mathbf{n m})\end{array}$ \\
\hline $\mathrm{A}$ & 16.2 & 0.21 & 0.84 & 0.56 & $85 \pm 8.9$ \\
$\mathrm{~B}$ & 15.9 & 0.21 & 0.84 & 0.74 & $107 \pm 9.5$ \\
$\mathrm{C}$ & 15.7 & 0.21 & 0.84 & 0.91 & $135 \pm 11.2$ \\
$\mathrm{D}$ & 13.4 & 0.21 & 0.84 & 2.22 & $83 \pm 51.2$
\end{tabular}

It was noted that increasing $\mathrm{NH}_{4} \mathrm{OH}$ concentration from $0.56 \mathrm{M}-0.91 \mathrm{M}(\mathrm{A}-\mathrm{C})$ increased nanoparticle size as noted by TEM analysis (Figure 3.5A-C) which shows that Samples A- 
C had uniform particles with average sizes of $85 \pm 8.9 \mathrm{~nm}, 107 \pm 9.5 \mathrm{~nm}$, and $135 \pm 11.2$ $\mathrm{nm}$. The same findings have been reported in the literature, ${ }^{56,132,135}$ indicating that particle size increases with increasing $\mathrm{NH}_{4} \mathrm{OH}$ concentrations at constant water and TEOS concentrations.
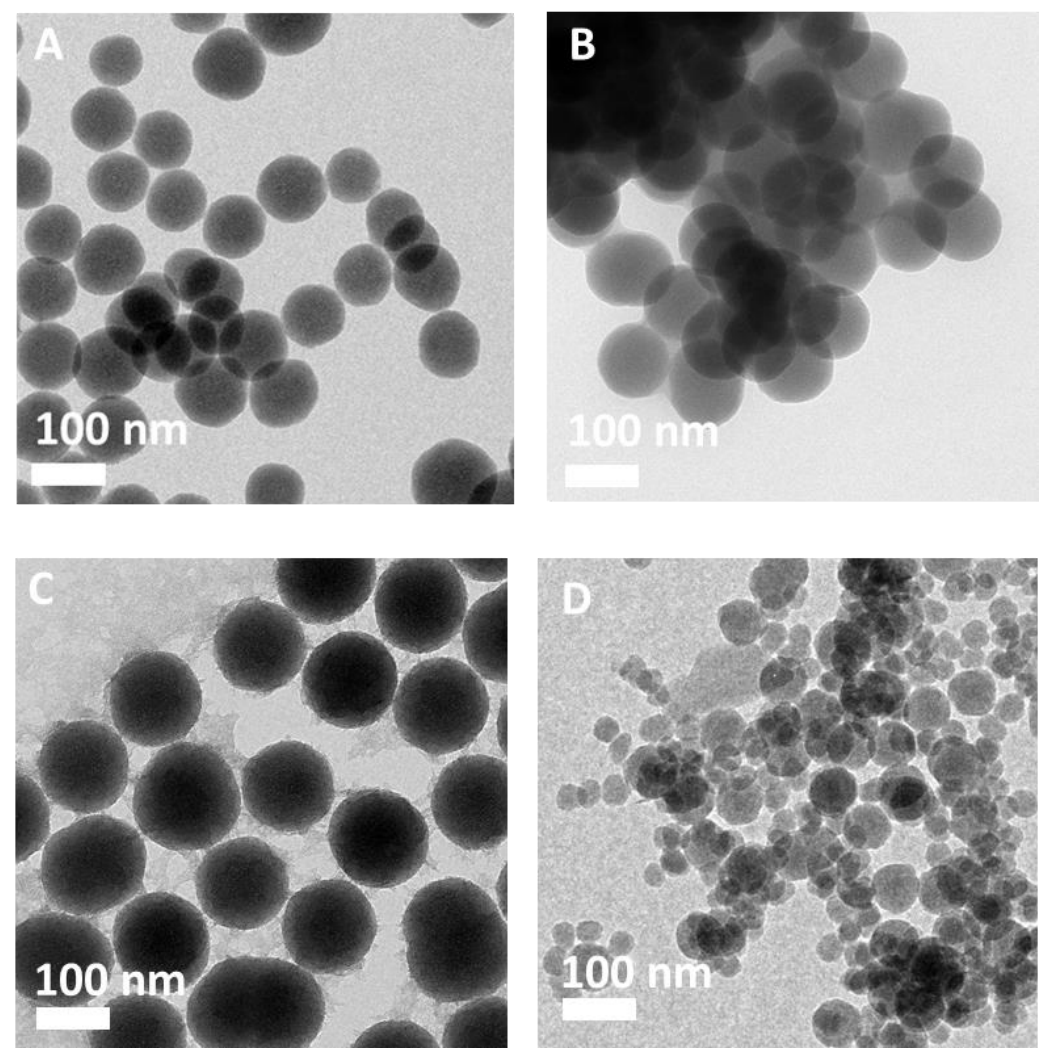

Figure 3.5 TEM images of nanoparticles synthesized according to the reagent amounts presented in Table 3.1

The observed trends are due to an increase in catalysis rate; as $\mathrm{NH}_{4} \mathrm{OH}$ concentration increases, it liberates many precursor molecules that increase the total energy of the systems and cause spontaneous aggregation to lower the energy levels, thereby accelerating polycondensation. Aside from that, $\mathrm{NH}_{4} \mathrm{OH}$ creates an environment with a much higher 
$\mathrm{pH}$ level above the isoelectric point, causing the silanol groups to be highly negatively charged and repel one another, critical in synthesizing nanoparticles with narrow size distribution (monodisperse).

To see if further increasing $\mathrm{NH}_{4} \mathrm{OH}$ concentration would maintain the same trend, a very high concentration (2.2 M) was used for the last sample (Figure 3.5D), and a decrease in size was noted in TEM $(83 \pm 51.2 \mathrm{~nm})$, indicating a wide size distribution. The findings are supported by the literature once more: the positive impact of ammonium concentration on size is observed between concentrations of $0.5 \mathrm{M}$ and $2 \mathrm{M}$. Beyond $2 \mathrm{M}$, the $\mathrm{NH}_{4} \mathrm{OH}$ negatively impacts size. The reasons for this observation have not been fully explained in the literature. Given the interactive effects of starting materials ${ }^{136}$, elucidating the interactive roles of starting material concentrations beyond the commonly used $\mathrm{NH}_{4} \mathrm{OH}$ concentrations may help explain the trend.

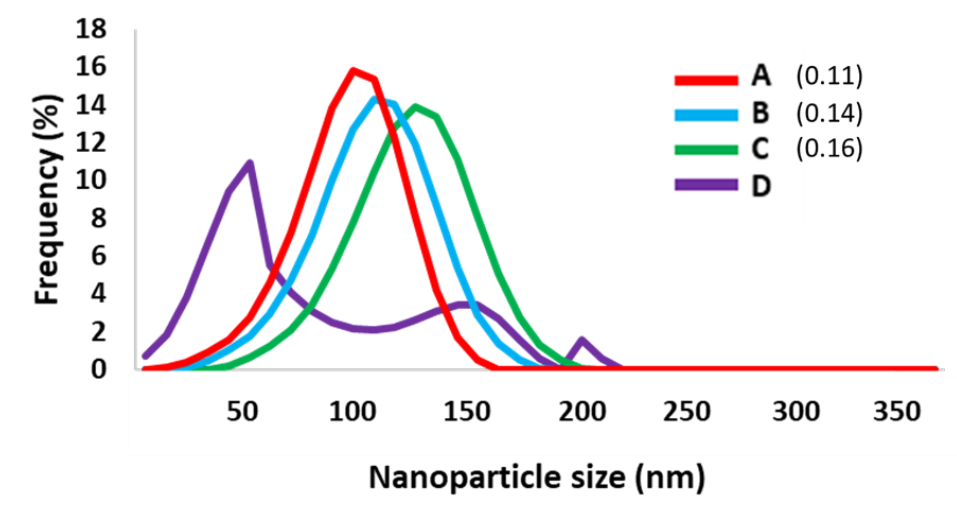

Figure 3.6 Supporting DLS size distributions of nanoparticles synthesized according to the reagent amounts presented in Table 3.1 
The TEM observations are further backed by DLS data (Figure 3.6) which shows that samples A-C have monomodal diameter sizes of $106.0 \mathrm{~nm}, 127.2 \mathrm{~nm}$, and $159.1 \mathrm{~nm}$ and polydispersity index $(\mathrm{PdI})$ values of $0.11,0.14$, and 0.16 while $\mathrm{D}$ had multimodal size distribution; hence the PdI could not be reliably determined. PdI values range from 0.0 (for a sample where all particles are the same size) to 1 (for samples with variable size populations). They can be calculated from DLS data using Eqn 3.1 below. Because PdI values less than 0.2 are presumably acceptable in practice and indicate highly homogenous samples ${ }^{137}$, the obtained PdI values show our synthesized nanoparticles are within the acceptable range of monodispersity.

$$
P d I=\left(\frac{\delta}{\text { mean }_{d}}\right)^{2}
$$

The link between $\mathrm{NH}_{4} \mathrm{OH}$ and the mean particle size was estimated using regression analysis with $\mathrm{r}$-values ranging from -1 to +1 . Values close to +1 indicate a strong positive linear association and values closer to - 1 suggest a strong negative linear relationship. Eqn 3.2 was used to calculate the r-value (correlation coefficient).

$$
\mathrm{r}=\frac{1}{n-1} \sum\left(\frac{x_{i}-\bar{x}}{S_{x}}\right)\left(\frac{y_{i}-\bar{y}}{S_{y}}\right)
$$

where $\mathrm{n}$ is the number of samples, $\bar{x}$ is the mean value of $\mathrm{x}$ variable, $\bar{y}$ is the mean value of $\mathrm{y}$ variable, and $\mathrm{S}_{\mathrm{x}}$ and $\mathrm{S}_{\mathrm{y}}$ is the standard deviation for $\mathrm{x}$ and $\mathrm{y}$ variables.

Figure 3.7 shows the analysis results. We conclude that the concentration $\mathrm{NH}_{4} \mathrm{OH}$ and mean values of synthesized NPs are positively correlated, with a correlation coefficient of 


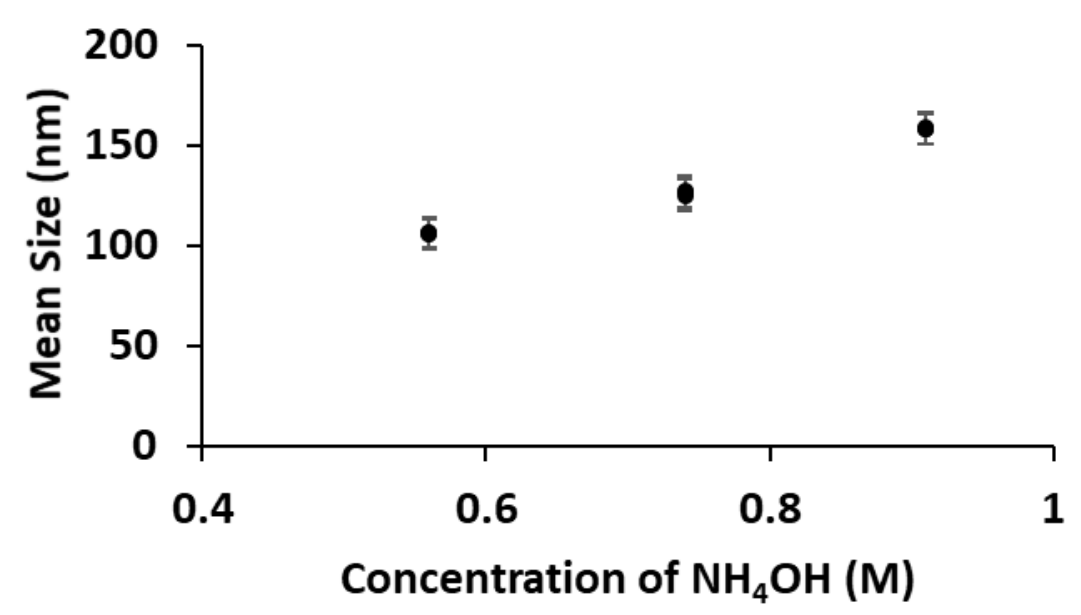

Figure 3.7 DLS results of solid $\mathrm{SiO}_{2}$ diameter as a function of $\mathrm{NH}_{4} \mathrm{OH}$ concentration

0.98 . With a p-value of 0.05 , the correlation coefficient of 0.98 is statistically significant. A high correlation means that these two variables have a strong relationship with each other. A positive correlation implies that the movement of two variables in the same direction. Thus, as the $\mathrm{NH}_{4} \mathrm{OH}$ concentration increases, the mean size of the nanoparticles increases as well. Such a linear correlation has also been reported in previous literature. However, the study stated that the relationship is linear until at about $0.4 \mathrm{M}$, after which the relationship becomes non-linear but increases before decreasing. ${ }^{138}$

\subsubsection{Fabricating the hMSNs}

The solid $\mathrm{SiO}_{2} \mathrm{NPs}(\mathrm{A})$ were then coated with a mesoporous silica shell by a modified Stöber method that utilized CTAB as the pore-forming agent. TEM analysis (Figure 3.8 A) showed the successful deposition of a silica shell approximately $20 \mathrm{~nm}$ in diameter. The outer hydrophilic (with the cationic head) surface of CTAB came in contact with both the 
hydrolyzed TEOS anions and negatively charged silanols of the solid $\mathrm{SiO}_{2}$ templates ensuring that condensation occurred on the surface. The hydrolysis, nucleation, and condensation proceed according to the process already explained for MSNs in Chapter 2. The only difference is that condensation takes place on the surface of the solid templates introducing a $\mathrm{CTAB} / \mathrm{SiO}_{2}$ hybrid layer onto the nanoparticles.
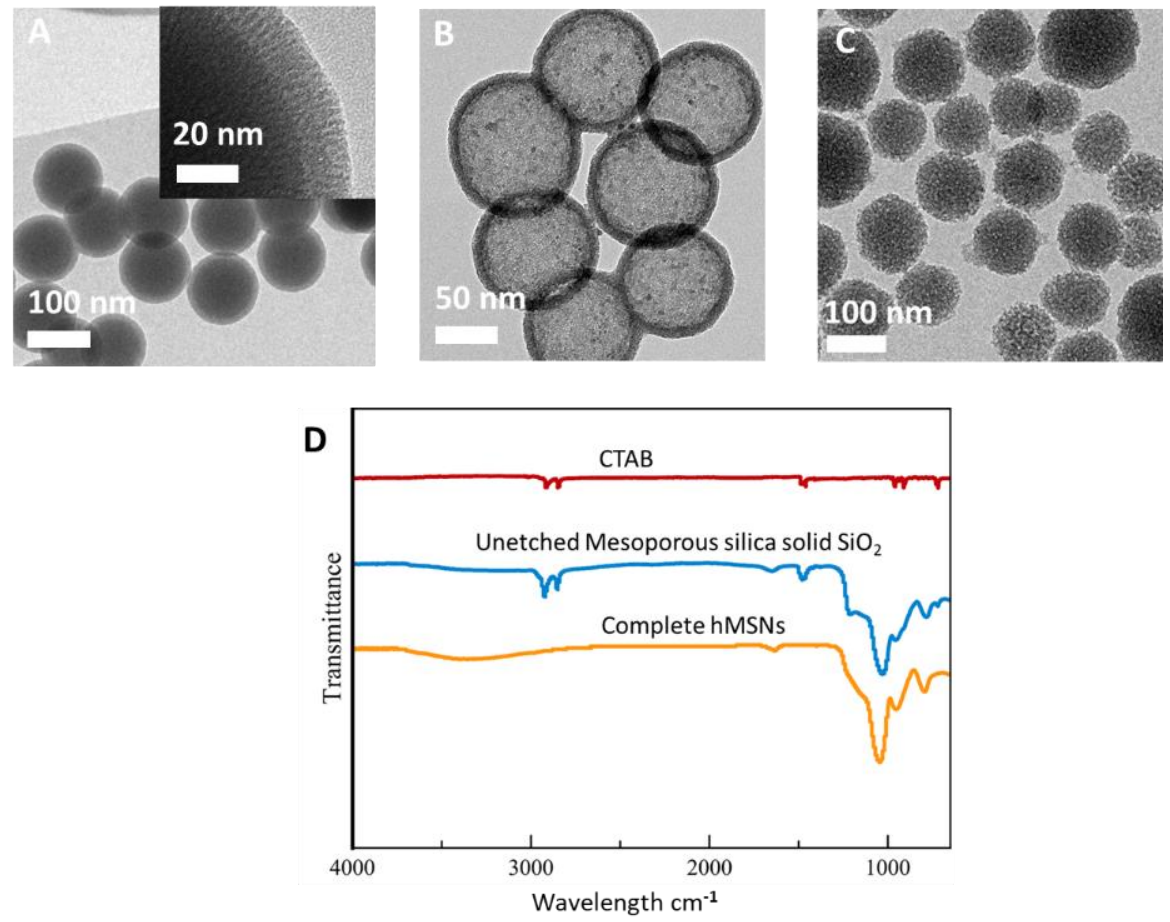

Figure 3.8 TEM images of A) Mesoporous shell coated ( 20nm) Solid $\mathrm{SiO}_{2}$ nanoparticles B) Core etched mesoporous coated Solid $\mathrm{SiO}_{2}$ nanoparticles $C$ ) Nanoparticle with etching order reversed D) Infrared transmission spectra of CTAB, mesoporous coated Solid $\mathrm{SiO}_{2}$ nanoparticles and complete hMSNs

The use of monodisperse silica templates allows for the replication of uniformity to the hMSNs. The presence of silanol also allows for interfacial recognition of the silicates, encouraging condensation on the surface and no other nuclei formation. ${ }^{77}$ The absence of small nuclei and uncoated solid $\mathrm{SiO}_{2}$ on the TEM image (Figure 3.8A) indicates the 
successful deposition of the shell and the prohibition of new nuclei. Furthermore, FTIR analysis (Figure 3.8D) of the mesoporous silica shell coated nanoparticles, when compared to the CTAB spectra (top spectrum), indicates the presence of peaks that are ascribed to CTAB: at about $2920 \mathrm{~cm}^{-1}$ and $2850 \mathrm{~cm}^{-1}$ which are caused by the asymmetric and symmetric vibrations $-\mathrm{CH}_{2}$ groups. ${ }^{98,99}$

The third stage involves using $\mathrm{Na}_{2} \mathrm{CO}_{3}$ for etching out the solid silica core through a selective structural procedure. Figure 3.8B depicts a completely cored etched nanoparticle with a negligible size change. The etching method relies on structural differences between the core and the shell. The mesoporous coating is said to undergo a higher degree of condensation due to the high synthesis temperature. It is further protected by the presence of $\mathrm{CTAB}$, while the core exhibits a lower level of condensation. When subjected to sodium carbonate etchant, the core is thus etched away relatively faster than the preserved mesoporous coating. The role of $\mathrm{CTAB}$ is thus not only to act as a soft template for pore formation but also to promote etching out of the core. ${ }^{139}$ This results in the core being etched relatively quickly than the preserved mesoporous silica since it allows etchant species $\left(\mathrm{OH}^{-}\right)$to penetrate.

To test this hypothesis, the etching order of the nanoparticles was reversed, and Figure 3.8 $\mathrm{C}$ shows the structural changes that occur when shell etching precedes core etching. There appears to be no directionality in the etching as the resultant TEM image shows wide size varieties. This might indicate that $\mathrm{CTAB}$ also controls the direction of etching, maintaining the size uniformity. Indeed, without the CTAB, the shell is equally vulnerable to the etchant 
and may well be etched even relatively faster given the increased surface area onto which dissolution might occur, leading to a much smaller TEM size than when the order is reversed.

Finally, the surfactant was removed using $\mathrm{EtOH} / \mathrm{HCl}$ etching as done for the MSN synthesis, and Figure 3.9 shows the complete hMSNs TEM images. The method relies on $\mathrm{H}^{+}$ions from the acid to interfere with the electrostatic attraction between the anionic

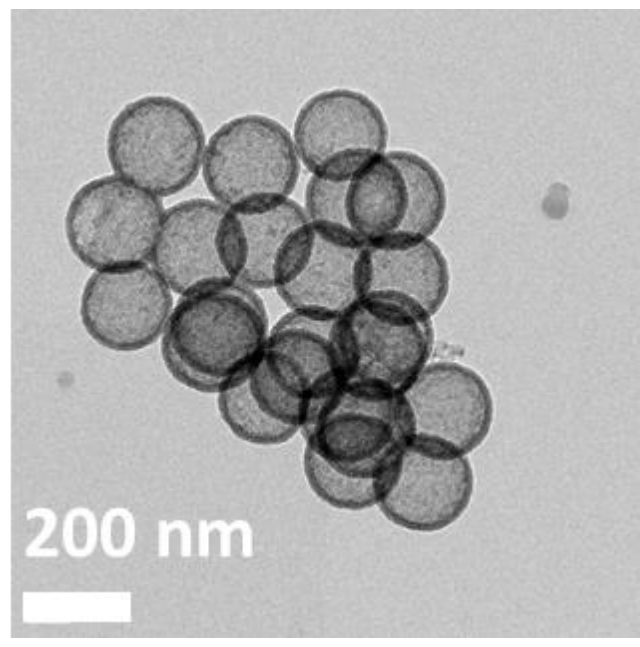

Figure 3.9 TEM image of complete hMSNs

silicates and the surfactant's cationic heads. The $\mathrm{H}^{+}$ions percolate through the entire silica network electrostatically exchanging with $\mathrm{CTA}^{+}$because the CTA+ and the silicates interactions at the interface are weaker than those with the small $\mathrm{H}^{+}$ions. ${ }^{140}$ Following successful extraction, CTAB peaks were missing (Figure 3.8C). The only peaks present were typical silica peaks: $\mathrm{Si}-\mathrm{O}-\mathrm{Si}$ asymmetric stretching at about $1020 \mathrm{~cm}^{-1}$ and $\mathrm{Si}-\mathrm{O}-\mathrm{H}$ asymmetric stretching around $960 \mathrm{~cm}^{-1} \cdot 95,141$

The size and distribution of the pores were measured using $\mathrm{N}_{2}$ porosimetry isotherms, and Figure 3.10 summarizes the findings. hMSNs exhibited the characteristic type IV isotherms 

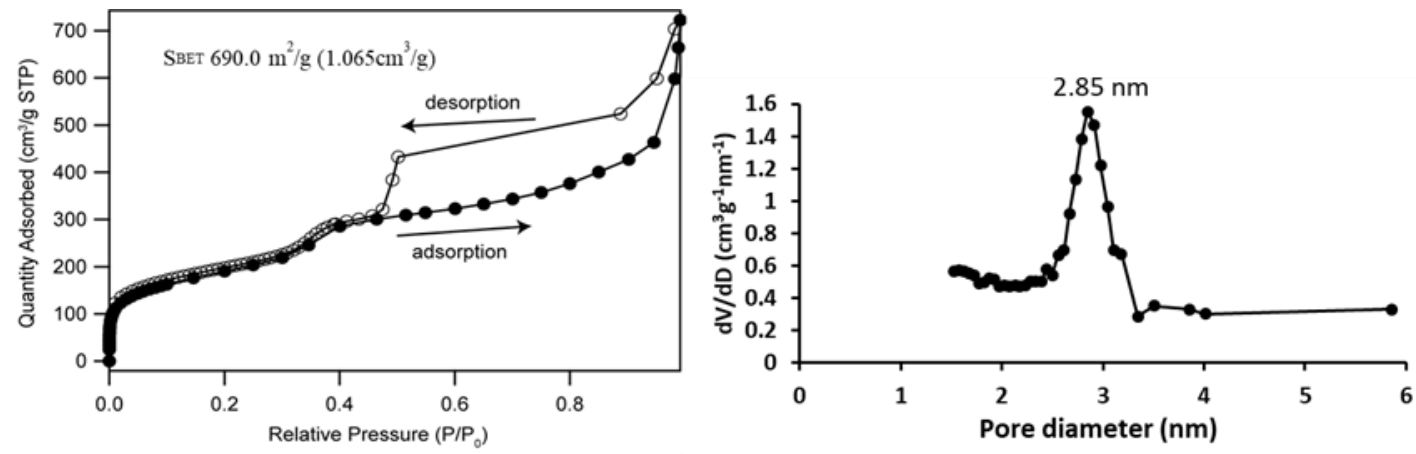

Figure 3.10 Left) $N_{2}$ porosimetry isotherm and Right) BJH pore size distribution curve for synthesized hMSNs (left) associated with primarily mesoporous material. Adsorption increases with increasing relative pressure at low relative pressures. At that time, the nitrogen molecules were adsorbed onto the inner surface of the sample's pores in single or multiple layers. When the relative pressure was gradually increased, the adsorption rose abruptly due to capillary condensation of $\mathrm{N}_{2}$ in the mesopores. Following that, the increase was moderate until saturation was reached. $\mathrm{S}_{\mathrm{BET}}$ for the hMSNs was determined to be $690.0 \mathrm{~m}^{2} / \mathrm{g}$. BJH pore size distribution curve for synthesized hMSNs is presented in Figure 3.10 (right) and reveals that hMSNs have a narrow pore size distribution that was peaked at $2.85 \mathrm{~nm}$.

\subsection{Conclusions}

This chapter documents the synthesis and characterization of hMSNs. To ensure reproducibility of hMSNs, a reliable synthesis procedure for solid template $\mathrm{SiO}_{2} \mathrm{NPs}$ needed to be developed. Besides the strict control of starting material concentration and conditions, the order and manner of reactant addition can further enhance the quality of synthesized nanoparticles. Procedure-A contributed to the better homogeneity of the TEOS 
within the starting solution, making it the mode of choice for synthesizing solid $\mathrm{SiO}_{2} \mathrm{NPs}$ with narrow size distributions. Different sized solid $\mathrm{SiO}_{2}$ were then synthesized by changing the base concentration, and the noted trends have been reported in the literature before. Procedure-A can be adopted as the standard method in the lab for continuously producing solid $\mathrm{SiO}_{2} \mathrm{NP}$ of different sizes.

The subsequent deposition of a mesoporous silica shell was done following a literature method with slight modifications, and TEM, FTIR spectroscopy, and $\mathrm{N}_{2}$ porosimetry confirmed the synthesis success. The role of CTAB in protecting the shell during core etching was demonstrated, and results may suggest that CTAB also offers directionality in etching. Overall, the synthesis yielded very repeatable nanoparticles, which were then functionalized, loaded, and evaluated as described in Chapter 4. 


\section{CHAPTER 4: LOADING, COATING, AND QUANTIFICATION OF SURROGATE DYE (ROSE BENGAL)}

\subsection{INTRODUCTION}

MSNs potentially have higher loading capacity than other nanocarriers, and substantial work has been done to further improve their loading capacities. The synthesis of MSN derivatives with increased void volume (hMSNs, as shown in Chapter 3) and the use of bulky silanes such as OTMS, APTES, and CPTES for functionalization and shape adjustment has been accomplished with a high level of complication. ${ }^{79}$ Because of the presence of numerous $\mathrm{Si}-\mathrm{OH}$ surface sites on $\mathrm{SiO}_{2} \mathrm{NPs}$, the surfaces are easily modifiable, allowing the MSNs to be functionalized appropriately to load various charged molecules via electrostatic interactions, hydrophilic/hydrophobic balance effects, and van der Waals forces. ${ }^{142}$

It has been previously shown that changing the type of functionalization can improve the loading capacity of ionic species by increasing the number of electrostatic attraction sites and the overall magnitude of surface charge. ${ }^{106}$ For example, She et al. increased the loading of 5-fluorouracil (5-FU) into hMSNs to $28.89 \%$ (weight) by surface functionalizing the NPs with amines from the $18.34 \%$ unmodified hMSNs. They credited the increase in loading to the electrostatic attractions between 5-FU and positively charged amino-modified hMSNs (due to the negatively charged groups on 5-FU and ammonium

groups on the hMSNs). ${ }^{143}$ Other research has also been conducted to increase the loading capacity of negatively charged ions into MSNs; these works record a significant increase 
in loading capacity (weight percent of payload) over unmodified samples. ${ }^{79,83,144}$

There are two main approaches through which functionalization has been achieved in MSN surface modifications in past work. Post-synthesis grafting attaches functional groups to the surface of MSNs after surfactant removal. In this method, the surface $\mathrm{Si}-\mathrm{OH}$ groups act as the anchoring points for organic functional groups. Silylation is the most frequent surface functionalization method with organic groups, which is accomplished through hydrolysis-condensation grafting of additional silane monomers. Alternatively, tetraalkoxysilane and one or more organoalkoxysilanes with at least one Si-C linkage can be used in situ co-condensation (during particle synthesis), which results in functionalization of the resulting mesoporous silicate nanostructures. ${ }^{145}$ However, unlike post-synthesis grafting, which results in an inhomogeneous distribution of the organic functional groups, in situ co-condensation (e.g., of TEOS and an APTES) within the NP synthesis step produces a more homogeneous distribution of organic groups.

Therefore, if uniform surface coverage with organic groups is desired in a single-step synthesis, the in-situ co-condensation method may be chosen. Suppose uniformity of functional groups is not an issue or is relatively less important $v s$. preservation of the porous structure (as in our case), the post-synthesis grafting method is usually preferred because bulky organoalkoxysilanes precursors often disrupt the natural textural qualities. Moreover, in situ co-condensation has been shown to increase the hydrodynamic radius of resulting amino-functionalized $\mathrm{SiO}_{2}$ particles significantly ${ }^{146}$ due to a greater tendency to aggregate. 
On top of surface functional group manipulations, additional post-synthesis coating and entrapment steps have been investigated for the effectiveness of increasing the amount of entrapped small molecule or ionic payloads in MSNs, hMSNs, and other types of nanoparticles with pores. ${ }^{147-149}$ Although encapsulation or coating of loaded NPs is frequently done to improve NP performance and increase circulation time in medical applications, ${ }^{150,151}$ some additional advantages of such steps include stabilizing the shape and structure of a nanomaterial, protecting an active surface or internal component from environmental impacts, separating compartments in a multi-component nanostructure, and adding new/additional functionalities. ${ }^{152}$ As previously indicated and supported in Chapters 2 and 3, we hypothesize that a thin silica coating on the NP's exterior surface should allow water exchange while entrapping the larger molecules (or ions) inside.

\subsection{EXPERIMENTAL}

\subsubsection{Materials}

Tetraethoxysilane 98\% (TEOS), Aminopropyltriethoxysilane 99\% (APTES), and methanol (reagent grade) were purchased from Sigma-Aldrich. Ammonium hydroxide $30 \%$ w/w aq. $\left(\mathrm{NH}_{4} \mathrm{OH}\right)$ and ninhydrin (ACS grade) were purchased from Fisher. Absolute EtOH 200 proof (absolute EtOH) was bought from Decon Labs, Inc., and electrophoretically pure $\mathrm{H}_{2} \mathrm{O}, 18 \mathrm{M} \Omega \bullet \mathrm{cm}$ resistivity $\left(\mathrm{mp} \mathrm{H}_{2} \mathrm{O}\right)$, was used for all synthesis protocols. Laboratory deionized water $\left(\mathrm{DI}-\mathrm{H}_{2} \mathrm{O}\right)$ and ethanol $(\mathrm{EtOH}$, reagent grade) was used for all washing steps. Experiments were performed in triplicates and representative samples, the mean \pm standard deviation was reported. 


\subsubsection{Thermogravimetric analysis (TGA)}

The TGA was done using a Mettler TA3000 thermal analysis system consisting of a TG50M3 thermobalance controlled by a TC10 TA processor. The data from the analysis was extracted from the processor via a LabVIEW script. The measurements were done on assynthesized std-MSNs and hMSNs to estimate silanol number (number of silanol groups per $\mathrm{nm}^{2}$ ) using approximate sample sizes of 5-10 $\mathrm{mg}$. The temperature was increased from $50{ }^{\circ} \mathrm{C}$ to $700{ }^{\circ} \mathrm{C}$ at a $10{ }^{\circ} \mathrm{C} / \mathrm{min}$ rate during sample measurement. To normalize the data for comparisons, the raw TGA data is expressed as a percentage of $\left(\mathrm{m}_{\text {initial }}-\mathrm{m}_{\text {final }}\right) / \mathrm{m}_{\text {initial }}$, with $m_{\text {initial }}$ and $m_{\text {final }}$ being the starting masses and mass at individual temperatures measurement during the experiment, respectively.

\subsubsection{Amine-functionalization of MSNs}

In a typical surface modification procedure, $50 \mathrm{mg}$ of the as-synthesized MSNs were dispersed in $3.27 \mathrm{~mL}$ of methanol using ultrasonication for 30 minutes, resulting in a milky suspension. Then, a pre-determined volume of APTES was then added. At room temperature, the mixture was stirred for 24 hours to complete the surface functionalization of MSNs. Afterward, the mixture was centrifuged for 15 minutes at $18000 \mathrm{rpm}$, and the resulting amine-functionalized NP solid was washed twice with $\mathrm{DI}-\mathrm{H}_{2} \mathrm{O}$ and twice with methanol and oven-dried overnight at $50^{\circ} \mathrm{C}$.

\subsubsection{Loading and coating}

In a $50 \mathrm{~mL}$ round bottom flask, $20 \mathrm{mg}$ of dry MSNs were suspended using sonication in 
$20 \mathrm{~mL}$ of an aqueous Rose Bengal $(10 \mathrm{mg} / \mathrm{mL})$ solution for $30 \mathrm{mins}$; the initial $\mathrm{pH}$ of this solution was not determined. This MSN-RB dispersion was stirred for 24 hours, and at the end of this time, the $\mathrm{pH}$ was adjusted to approximately 9 using $\mathrm{NH}_{4} \mathrm{OH}$. A pre-determined volume of TEOS was then mixed with an equal amount (volume) of absolute EtOH and added dropwise to the stirring MSN-RB suspension; the suspension was subsequently left to stir overnight. Afterward, centrifugation was used to collect solid NPs, then resuspended in $\mathrm{DI}-\mathrm{H}_{2} \mathrm{O}$ and centrifuged again. This procedure was repeated two more times with water. After each of the three washes, the supernatants were collected for spectroscopic quantification of the leached (from the MSNs) RB dye. The resultant pellet after the three washes and oven-dried overnight at $50{ }^{\circ} \mathrm{C}$.

\subsubsection{Characterization of loaded and coated MSNs}

The size, morphological and compositional features of the functionalized, loaded, and coated MSNs were analyzed by TEM and EDS using a Titan TEM with ChemiSTEM capability with Bruker SDD detectors (Oregon State University). All sample preparations for TEM and EDS, FT-IR spectroscopy, and $\mathrm{N}_{2}$ porosimetry were done according to procedures and methodologies previously described in Chapters 2 and 3.

\subsubsection{Amine quantification}

A spectroscopic ninhydrin assay was used to quantify the number of surface amines of functionalized MSNs (per $\mathrm{nm}^{2}$ of surface area). This method is primarily a biochemistry method for the detection of amine groups. In our case, the ninhydrin reagent oxidizes and deaminates the amino group from the surface of MSNs. Because two molecules of 
ninhydrin (2, 2- dihydroxyindane-1, 3-dione) react with the amino group to form a deep purple compound, the intensity of the formed complex is proportional to the concentration of amino groups in the solution. The resulting dye-to-amine group ratio is known, approximate quantification of the amino groups is possible (1:1). After being shown to be as good as the previously used calibration compounds (octylamine and propylamine), APTES was used as a standard ${ }^{153}$ as follows; absolute EtOH was used to create a $50 \mu \mathrm{M}$ $(0.05 \mu \mathrm{mol} / \mathrm{mL})$ solution of APTES. In absolute EtOH, a $2 \% \mathrm{w} / \mathrm{w}$ ninhydrin solution was also prepared. Then, $2 \mathrm{~mL}$ of the APTES stock solution was serially diluted with EtOH to make standards (using $0.5 \mathrm{~mL}$ aliquots). To each solution (and to a solvent blank containing only absolute EtOH), $1 \mathrm{~mL}$ of the ninhydrin solution was added. These were then heated to $60{ }^{\circ} \mathrm{C}$ for 30 minutes.

After APTES calibration curve generation, oven-dried amine-functionalized materials were suspended in absolute $\mathrm{EtOH}$ and ultrasonically dispersed (for about an hour) for amine quantification. $2 \mathrm{~mL}$ of nanoparticle suspension $(10 \mathrm{mg} / \mathrm{mL})$ was mixed with $1 \mathrm{~mL}$ of ninhydrin solution. The heating and measurement procedures were performed according to the calibration standards. Where necessary, the samples were diluted to the appropriate concentration to not saturate the absorbance. The concentrations of amino groups were determined by comparing the readings to the APTES-ninhydrin calibration curve.

\subsubsection{Loading capacity}

UV-Visible spectroscopy was used to determine the concentration of RB in the NP. Before measuring a supernatant sample, a calibration curve was generated using Rose Bengal 
solutions of known concentrations and plotting the measured absorbance intensity (in arbitrary units) $v s$. concentration of RB. Each sample was analyzed in at least three different dilutions (prepared by adding small aliquots of water ( $\mathrm{pH} 6.0$ - 6.5)). Absorbance intensity measurements at $546 \mathrm{~nm}$ were made on the supernatants taken from the NP samples washing steps. By comparing the readings to the standard curve, the concentration of RB leached from the particles is determined; $\mathrm{RB}$ that is not leached into the supernatants is assumed to be still associated with the particles.

\subsection{RESULTS AND DISCUSSION}

\subsubsection{Quantification of surface hydroxyls using TGA}

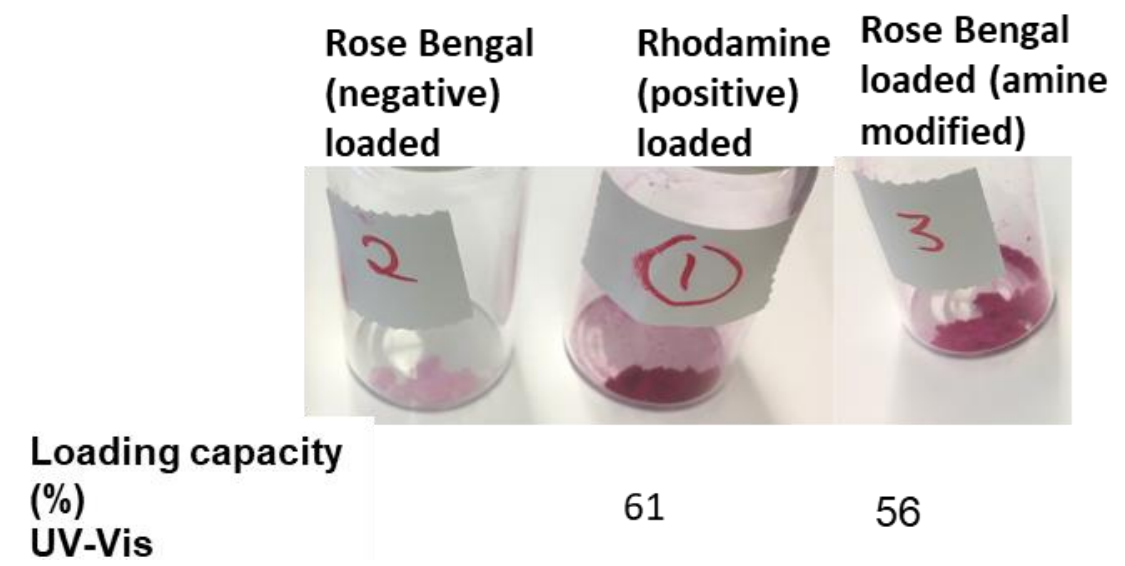

Figure 4.1 Effect of surface charge on the loading capacity of cationic vs. anionic dyes.

The silica surface adsorptive properties influence the adsorption of molecules in mesoporous matrices. As a result, the molecular or ionic payload associated with a porous silica NP is expected to be determined by chemical interactions between the functional groups covering the silica NP surface and the functional groups of the guest molecule (or 
ions). For as-prepared mesoporous silica NPs, the surface is negatively charged at nearneutral $\mathrm{pH}$ due to deprotonated silanol groups.

We demonstrated in preliminary data that Rhodamine, a positively charged dye, loads more efficiently than RB (negative) in unmodified mesoporous silica nanoparticles (Figure 4.1) (61\% loading capacity vs. 9\%). To increase the loading capacity of RB, we functionalized the surface with positive amine groups, which resulted in an increase in the loading capacity of RB from (9\% to $56 \%$ ).

We thus amine-functionalized the MSN and hMSN particles prepared in earlier chapters to increase the RB's loading capacity. APTES is an organic silane with one amine functional group and three hydrolyzable ethoxy groups that permit attachment to the silanol surface; we hypothesized that the amine functional groups would be protonated to some extent, which should reduce charge repulsions between RB (negatively charged) and the nanoparticles (initially negatively charged).

In the surface reaction between APTES and the MSNs (or hMSNs), the hydrolyzable ($\left.\mathrm{OCH}_{2} \mathrm{CH}\right)_{3}$ groups of APTES are first hydrolyzed to - $\mathrm{OH}$ groups and then condensed with free silanol groups at the MSN surface, resulting in overall positively charged MSNs once resuspended in aqueous solution. Additionally, we chose conditions where APTES would not be expected to fully cover the silanol surface groups. The surface functionalization needed to spare some hydroxyls onto which the subsequent deposition of a thin solid $\mathrm{SiO}_{2}$ shell could be deposited. In order to approximate the number of silanols on the surface, a TGA method was used. Figure 4.2 shows the representative thermogravimetric curves of 
std-MSN and hMSNs before amine modification.

TGA graphs of silicas generally show two steps (characterized by a change in slope steepness); Step 1, removing physically adsorbed water at $200{ }^{\circ} \mathrm{C}$ and below, ${ }^{154,155}$ which corresponds to our findings of a temperature range of 50 to $190{ }^{\circ} \mathrm{C}$ for physically adsorbed water removal and Step $2\left(>200^{\circ} \mathrm{C}\right)$ shows weight loss from the powder surface due to the

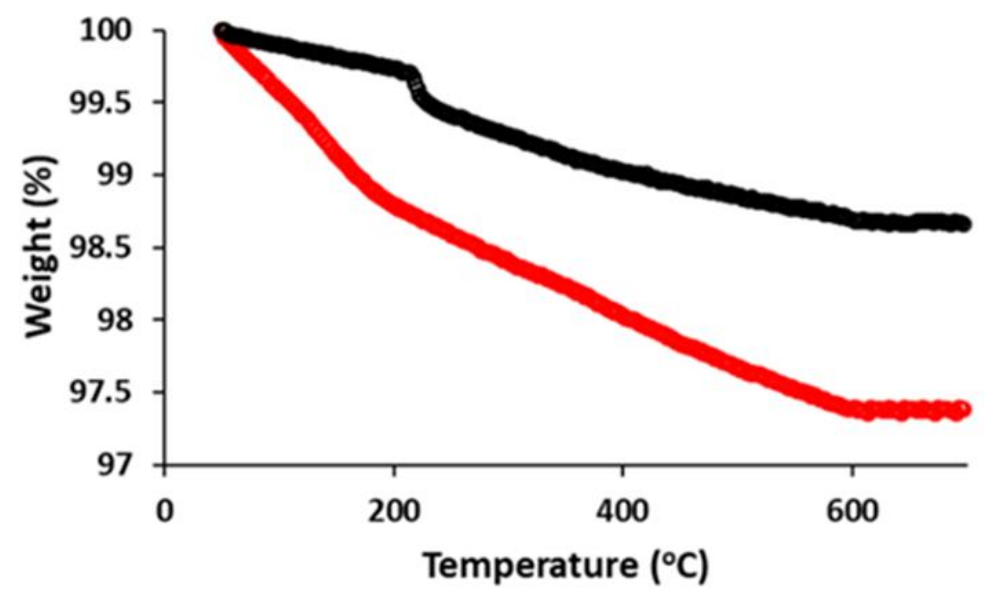

Figure 4.2 Thermogravimetric analysis (TGA) curves for std-MSNs (Black) and hMSNs (red)

elimination of silanol groups. ${ }^{154} \mathrm{hMSNs}$ appear to have a higher quantity of adsorbed water molecules (approximately $1.2 \%$ compared to $0.3 \%$ for std-MSNs) despite the two samples being dried under identical conditions before analysis. Literature has since explained that such differences are typical since the water content can be affected by humidity during sample preparation. ${ }^{156}$ Using data from TGA experiments, the Eqn 4.1 below can be used to determine the number $\mathrm{nOH}$ per $\mathrm{nm}^{2}$ of the samples:

$$
n_{O H=\frac{m_{T G A \times N_{A}}}{M_{W} S_{B E T}}}
$$


Where $m_{T G A}\left(\mathrm{~g} \mathrm{~g}^{-1}\right)$ is the mass loss due to $-\mathrm{OH}$ occurring at temperatures above $200{ }^{\circ} \mathrm{C}$, $\mathrm{N}_{\mathrm{A}}$ is the Avogadro's number $\left(\mathrm{mol}^{-1}\right), \mathrm{MW}_{\mathrm{W}}\left(\mathrm{g} \mathrm{mol}^{-1}\right)$ is the molecular weight for the silanol group, and $\mathrm{S}_{\mathrm{BET}}\left(\mathrm{m}^{2} \mathrm{~g}^{-1}\right)$ is the surface area of the sample obtained from $\mathrm{N}_{2}$ porosimetry experiments.

Table 4.1 summarizes the results for silanol numbers of the samples with hMSNs calculated to have $1.34 \mathrm{~nm}^{2}$ while std-MSNs have $1.02 \mathrm{~nm}^{2}$.

Table 4.1 Silanol number calculations for std-MSNs and hMSNs

\begin{tabular}{|l|l|l|l|}
\hline Sample & $\mathrm{S}_{\mathrm{BET}}\left(\mathrm{m}^{2} \mathrm{~g}^{-1}\right)$ & $m_{T G A}\left(\mathrm{~g} \mathrm{~g}^{-1} \mathrm{~g}\right)$ & $\begin{array}{c}\text { Silanol number } \\
\left(\text { per } \mathrm{nm}^{2}\right)\end{array}$ \\
\hline Std-MSNs & $1198.8 \pm 4.5$ & 1.07 & 1.02 \\
\hline hMSNs & $690.02 \pm 1.44$ & 1.40 & 1.34 \\
\hline
\end{tabular}

Details of $\mathrm{S}_{\mathrm{BET}}$ characterization are given in the subsequent sections

\subsubsection{Amine modification of MSNs}

In order to investigate the feasibility of this, 3 different amounts of APTES (meant to functionalize $25 \%, 50 \%$, and $75 \%$ of the total silanols) were used to treat $50 \mathrm{mg}$ sample std-MSNs. The FTIR analysis was used to analyze the NPs functional groups and observe the effect of the APTES concentration on the silica surface. As-synthesized and surfacemodified MSNs were then characterized using FTIR (Fig 4.3) to confirm the incorporation of the different functional groups onto the MSNs' surface. The spectrum of the assynthesized MSNs and all the modified samples (B-E) peaked around $1020 \mathrm{~cm}^{-1}$ and $960 \mathrm{~cm}^{-1}$ due to $\mathrm{Si}-\mathrm{O}-\mathrm{Si}$ asymmetric stretching vibrations and asymmetric Bending stretching vibration Si-OH, respectively. After APTES functionalization, the $\mathrm{CH}_{2}$ 


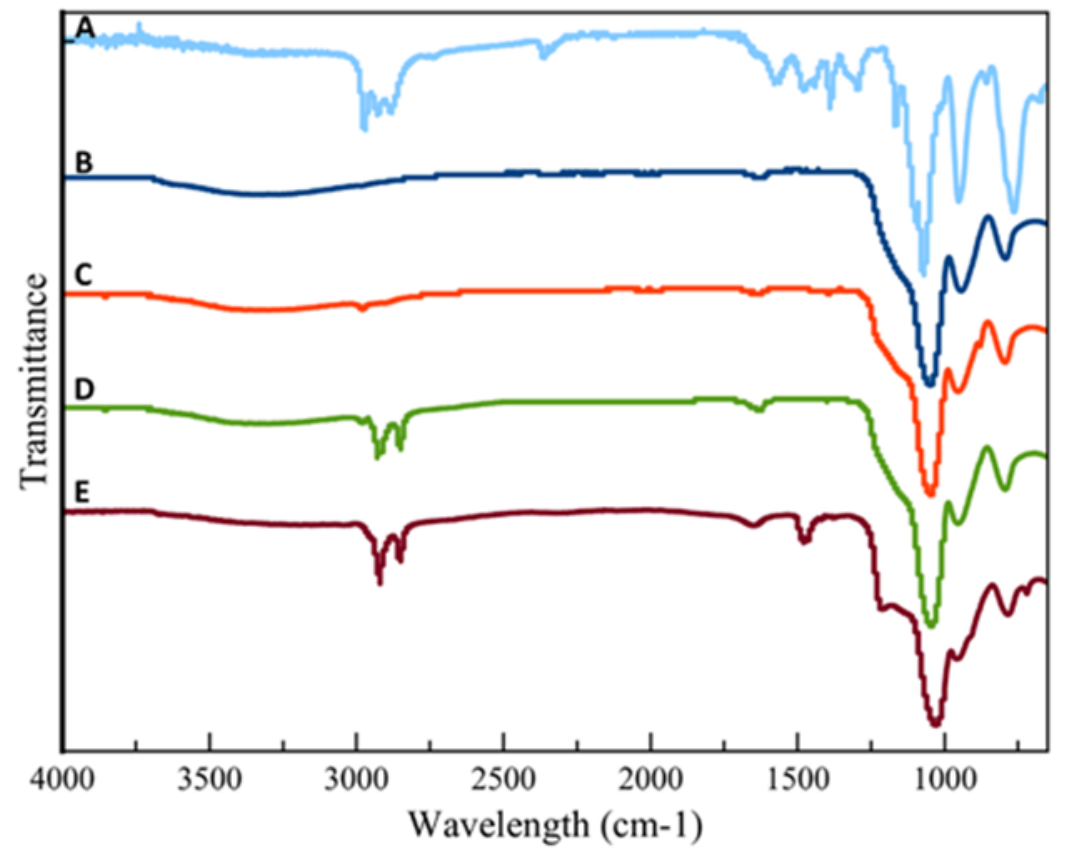

Figure 4.3FTIR spectra of A) APTES, B) as-synthesized silica and amine modified std-MSNs with different amounts of APTES $(C-E)$

asymmetric and symmetric stretching modes at roughly $2932 \mathrm{~cm}^{-1}$ and $2864 \mathrm{~cm}^{-1}$, respectively, indicate the successful introduction of amines onto the surface of std-MSNs. The presence of the $\mathrm{NH}_{2}$ group from APTES was shown by the two labeled peaks appearing at roughly $1600 \mathrm{~cm}^{-1}$, and belonging to the $\mathrm{NH}_{2}$ scissor vibrations is also evidence of successful grafting.

Interestingly, as the amount of APTES was increased, the peaks accredited to APTES became more and more pronounced. Despite the peaks in Sample $\mathrm{C}$ not being as pronounced, zeta potential measurements revealed that the particles had a surface charge of $12.6 \mathrm{mV}$ compared to $13.5 \mathrm{mV}$ for the as-synthesized samples. hMSNs were also treated with just enough APTES to cover approximately $25 \%$ of the surface based on theoretical 
calculations from using $S_{\mathrm{BET}}$ and TGA results.

The amine-modified samples were analyzed using $\mathrm{N}_{2}$ porosimetry to investigate the effect of functionalization on surface area and volume. Figure 4.4 shows the $\mathrm{N}_{2}$ porosimetry findings, and Table 4.2 summarizes the major findings. The std-MSNs and hMSNs (assynthesized and amine-modified) have a predominantly mesoporous structure, typical of Type IV mesoporous materials. A monolayer to multilayer adsorption mechanism on the mesopore walls is indicated at a lower relative pressure $\left(\mathrm{P} / \mathrm{P}_{0}<0.4\right)$. At pressures less than the saturation vapor pressure, the gas condenses into bulk liquid in the mesopores at higher relative pressures, giving rise to the observed hysteresis loop, which is more pronounced in the hMSNs, presumably due to the presence of the internal void.
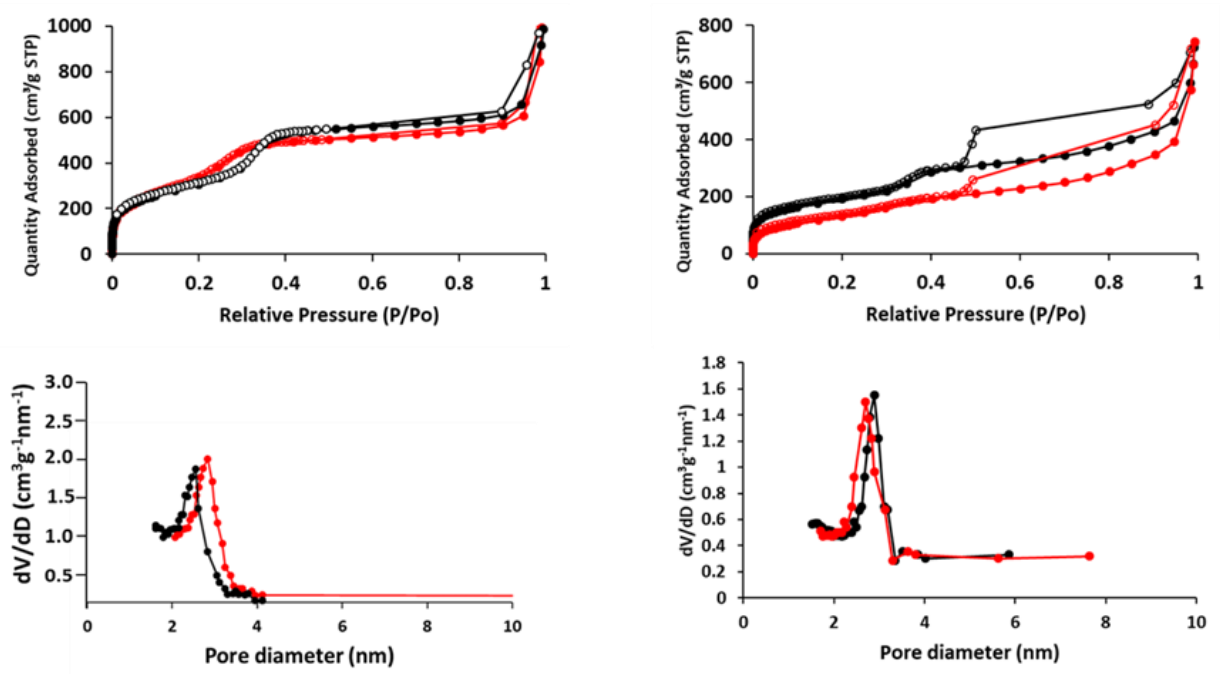

Figure 4.4 $N_{2}$ porosimetry studies showing isotherms (Top) and pore size distribution (Bottom) of MSNs (Left column) and h-MSNs (Right). Black shows the as-synthesized nanoparticles, and red shows the aminemodified samples.

The $\mathrm{S}_{\mathrm{BET}}$ of std-MSNs, $1198.8 \mathrm{~m}^{2} \mathrm{~g}^{-1}$, went down to $1113.2 \mathrm{~m}^{2} \mathrm{~g}^{-1}$, while that of hMSNs went down from $690.0 \mathrm{~m}^{2} \mathrm{~g}^{-1}$ to $676.8 \mathrm{~m}^{2} \mathrm{~g}^{-1}$ following amine functionalization. These 
Table 4.2 Summary of $\mathrm{N}_{2}$ porosimetry experiments

\begin{tabular}{lll} 
Sample & $\mathbf{S}_{\text {BET }}\left(\mathbf{m}^{\mathbf{2}} / \mathbf{g}\right)$ & $\begin{array}{l}\text { Pore diameter } \\
(\mathbf{n m})\end{array}$ \\
\hline Std-MSNs & $1198.8 \pm 4.5$ & 2.72 \\
NH-MSNs & $1113.18 \pm 3.76$ & 2.56 \\
hMSNs & $690.02 \pm 1.44$ & 2.84 \\
NH-hMSNs & $676.79 \pm 2.42$ & 2.62
\end{tabular}

changes were caused by a possible decrease in the surface area caused by adding amine groups. The pore sizes of the modified MSNs are nearly the same as that of the unmodified std-MSNs as summarized in Table 4.2, while hMSNs had a larger decrease (MSNs (decreases of $5.8 \%$ for std-MSNs and $7.7 \%$ for hMSNs). The particles needed to retain as much loading volume as possible; thus, a limited amount of APTES was used, which in both cases did not result in a drastic reduction of volume and surface area.

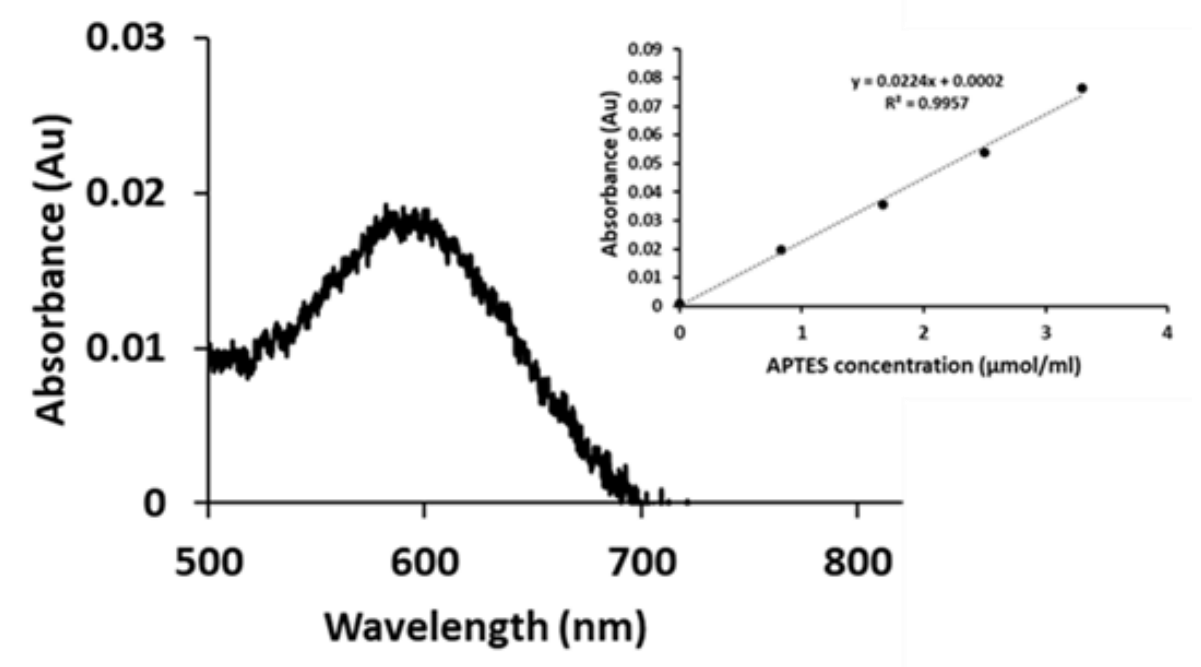

Figure 4.5 Ninhydrin standard curve for amine concentration determination

The surface coverage of amines on the surface was determined using the ninhydrin test via 
the APTES calibration curve shown in Figure 4.5 after Hristov et al. demonstrated that APTES could be used as standard with fair results. ${ }^{153}$ We also chose APTES as our standard since it is the amine modification agent we used in our surface modification. The obtained concentrations and $\mathrm{S}_{\mathrm{BET}}$ were used to estimate the surface amine surface coverage of StdMSNs and hMSNs, which were $0.232 \mathrm{~nm}^{-2}$ and $0.329 \mathrm{~nm}^{-2}$, respectively. These values are slightly lower than the anticipated levels of $0.255 \mathrm{~nm}^{-2}$ and $0.332 \mathrm{~nm}^{-2}$ based on the used amounts of TEOS. We speculated this could be due to functionalization being a diffusionlimited process; thus, surface silanol groups are more easily accessible than the interior pore surface silanols.

\subsubsection{Loading and coating}

A one-pot loading procedure was developed and used to load and trap RB (surrogate dye), with the loading quantified and the efficiency determined using UV-Vis spectroscopy.

While mesoporous silica coatings have been used in different types of nanoparticles, applying a silica coating requires careful consideration of starting material concentrations and the attempt to do it in a one-pot synthesis with compounds to the anticipated challenges. The main goal of developing the coating was to show that a silica shell could be deposited and effectively trap RB without initiating aggregation and self-nucleation. We needed to use the exact amount of TEOS in the coating procedure. We used Eqn 4.2 to estimate the amount of TEOS required for successful coating deposition based on our extensive knowledge of working with silica.

We calculated the mass of the required $\mathrm{SiO}_{2}$ coating layer $\left(\mathrm{m}_{\text {coat }}\right)$ ): 


$$
m_{(\text {coat })}=\rho_{\mathrm{SiO}_{2}} \times N_{\mathrm{SiO}_{2}} \times V_{(\text {coat })}
$$

Where $\rho_{\mathrm{SiO}_{2}}$ is the silica density, $N_{\mathrm{SiO}_{2}}$ is the number of nanoparticles in the loading suspension that was calculated from density and cumulative pore volume from $\mathrm{N}_{2}$ porosimetry studies and $V_{(\text {coat })}$ is the coating volume found by subtracting the uncoated nanoparticles' (based on TEM size) from the anticipated total volume.

Because the amount of TEOS required is equal to the amount of $\mathrm{SiO}_{2}$ formed, the volume of TEOS to be added was calculated from TEOS's density and molar mass.

The modified std-MSNs and hMSNs were incubated overnight in an RB solution

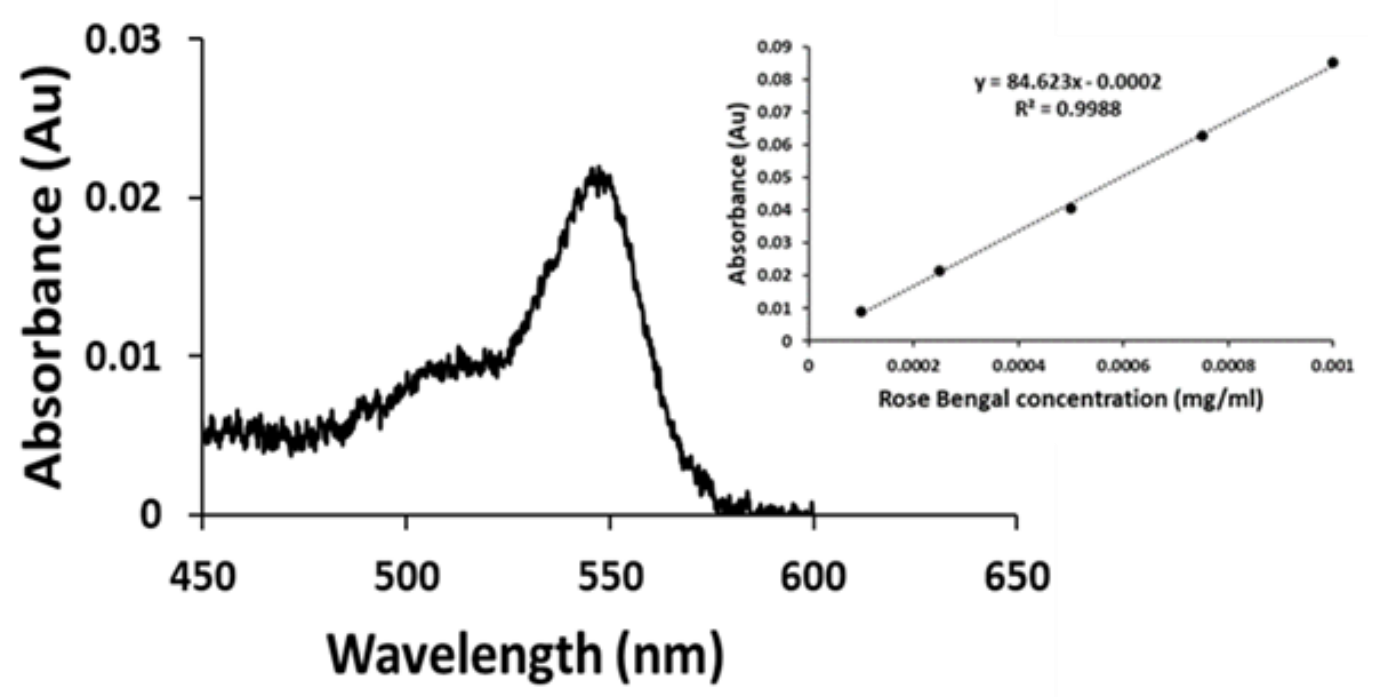

Figure 4.6. Standard curve for determining the loading capacity

$(1 \mathrm{mg} / \mathrm{mL})$ before the reaction conditions were adjusted, and pre-determined amounts of TEOS were added for the silica coating. Table 4.3 summarizes the six different determined loading capacities using the calibration standard of RB shown in Figure 4.6. experiments, including the volumes of TEOS used in the coating experiments. 
Table 4.3 Summary of loading and coating experiments.

\begin{tabular}{|l|l|l|l|l|l|l|}
\hline \multirow{2}{*}{ Samples } & \multicolumn{3}{|l|}{ Amine modified hMSNs } & \multicolumn{3}{c|}{$\begin{array}{c}\text { Amine modified std- } \\
\text { MSNs }\end{array}$} \\
\cline { 2 - 8 } & A & B & C & D & E & F \\
\hline Volume of TEOS $(\mu \mathrm{L})$ & 0 & 176 & 352 & 0 & 28 & 56 \\
\hline LC $(\%)$ (triplicates mean) & 12.0 & 71.4 & 70.9 & 11.1 & 31.0 & 58.1 \\
\hline Thickness of coating $(\mathrm{nm})$ & 0 & 7.25 & 7.14 & 0 & 3.15 & 9.91 \\
\hline
\end{tabular}

Assuming electrostatic interactions only, std-MSNs and hMSNs would have had loading capacities of $4 \%$ and $3,15 \%$, respectively, as determined by the reported amine densities in Section 4.3.1 and surface area studies.

The loading capacities of all samples (A-E) were much greater than the efficiency predicted by the amine quantification and surface area analyses (almost 3 times and 4 times more for std-MSNs and hMSNs, respectively). We anticipated that this value would be approximately determined by the amine density on the surface in an uncoated sample due to electrostatic forces being the only forces at work. Values that are nearly double the predicted value may, in addition to the apparent electrostatic forces, reflect the presence of non-electrostatic forces of attraction during the RB's entrapment within the pores. Thus, we were incorrect in thinking that electrostatic and covalent forces hold loading molecules within pores without a coating. hMSNs appear to have a greater loading capacity than MSNs, particularly among coated samples, averaging (71.4\%t, $70.9 \%$, compared to 31.0 $\%$, and $58.1 \%$, respectively), which could be owing to the additional void in hMSNs despite their smaller surface area.

TEM determined the thicknesses size of the coating layer, and Figure 4.7 shows the representative images. Coated samples B, C, E, and F, had thicknesses of $7.25 \mathrm{~nm}, 7.14$ $\mathrm{nm}, 3.15 \mathrm{~nm}$, and $9.91 \mathrm{~nm}$, respectively, with noticeably closed pores compared to 

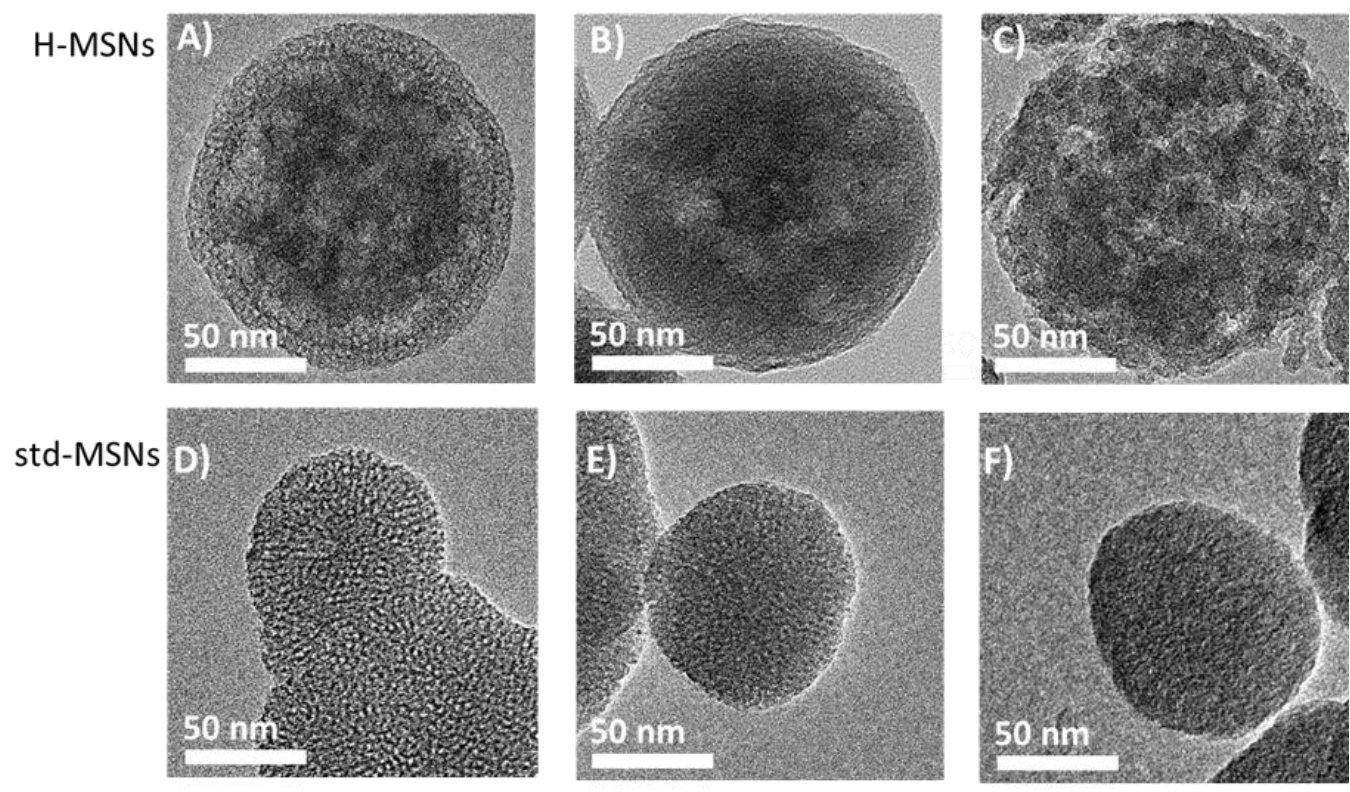

Figure 4.7 TEM images of the different samples presented in Table 4.3

untreated samples (A and D). In coating trials with hMSNs, increasing the TEOS content did not appear to appreciably increase the coating thickness. Excess TEOS appears to have collected as individual particles (perhaps due to secondary nucleation) or on the surface of the hMSNs, leading to their roughness. On the other hand, the two TEOS concentrations appear to have contained nearly the same amount of RB, which we reasoned was because the thickness was sufficient to properly encapsulate the chelate in both cases.

Coated std-MSNs exhibit a range of thicknesses and loading capacities of the RB. Sample C coating thickness was roughly $3.15 \mathrm{~nm}$ thick, whereas Sample D was $9.91 \mathrm{~nm}$ thick. There was a noticeable difference in the appearance of the pores in $\mathrm{E}$ when compared to the uncoated Sample D. However, the low LE value may indicate that such a thin layer is insufficient to effectively encapsulate RB in the pore. This, we believed, may account for 
the low LE as the RB continued to seep out during the washing phase. Doubling the TEOS concentration resulted in a significantly more irregular visual coating of the MSNs and increased coating toughness (Sample F). As evidenced by the higher LC, this resulted in a covering that effectively enclosed RB (31\% to $58 \%)$.
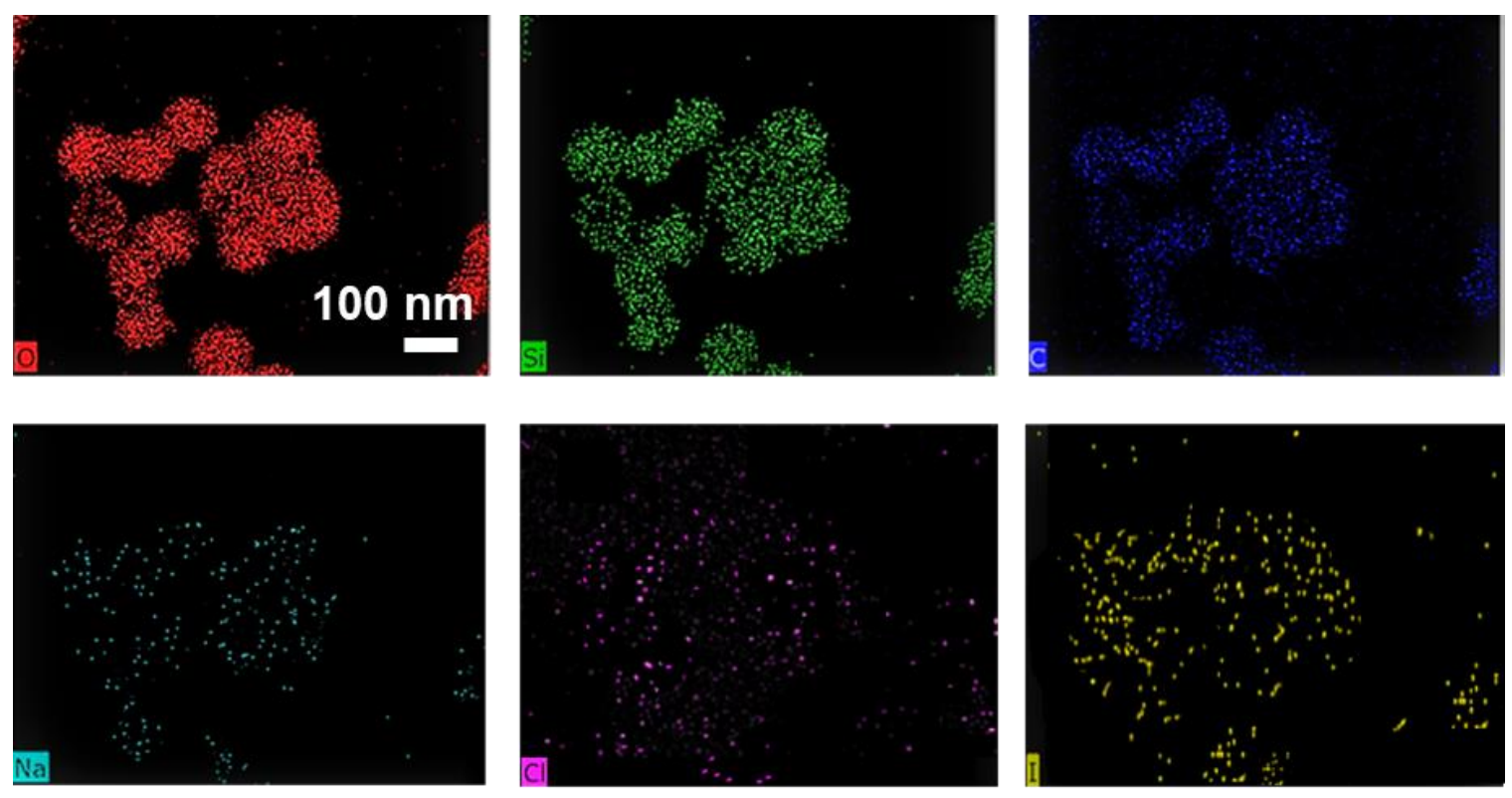

Figure 4.8 Elemental mapping of sample loaded MSNs $\left(B_{1}\right)$

The RB's location was also confirmed via elemental mapping, and as an example, Figure 4.7 illustrates the confinement of RB into the pores of hMSNs. Although the elemental mapping experimental settings are the same for samples A-F in terms of the amount of TEOS employed, the concentrations of the samples provided in Table 4.3 were too low for EDX mapping analysis. Relatively few counts were observed, and in some cases, were undetectable with large standard error in the gathered data. For this set of studies, a very high $\mathrm{RB}(100 \mathrm{mg} / \mathrm{mL})$ dosage was used instead, and the samples are denoted $\left(\mathrm{A}_{1}-\mathrm{F}_{1}\right)$. The representative map in the Figure illustrates the percentages of $\mathrm{Si}, \mathrm{O}, \mathrm{C}, \mathrm{Na}, \mathrm{Cl}$, and $\mathrm{I}$ in $\mathrm{B}_{1}$; 
the first two are derived mainly from the $\mathrm{SiO}_{2}$ host, whereas the latter are derived from $\mathrm{RB}$ molecules. The findings of the elemental mapping presented in Figure 4.8 and the summary of all samples are summarized in Table 4.4.

Table 4.4 Elemental mapping of loaded MSNs showing (atomic \%)

\begin{tabular}{|l|l|l|l|l|l|l|}
\hline Sample & $\mathrm{Si}$ & $\mathrm{O}$ & $\mathrm{C}$ & $\mathrm{Na}$ & $\mathrm{Cl}$ & $\mathrm{I}$ \\
\hline $\mathrm{B}_{1}$ & 20.3 & 39.4 & 22.8 & 3.2 & 5.5 & 8.8 \\
\hline $\mathrm{C}_{1}$ & 25.2 & 51.5 & 20.3 & 0.54 & 0.95 & 1.51 \\
\hline $\mathrm{E}_{1}$ & 28.1 & 58.5 & 10.3 & 0.45 & 1.24 & 1.41 \\
\hline $\mathrm{F}_{1}$ & 23.5 & 50.6 & 16.0 & 1.98 & 3.91 & 4.01 \\
\hline
\end{tabular}

$\mathrm{A}_{1}$ and $\mathrm{D}_{1}$ had undetectable levels of elements despite the increased concentrations

Based on the elemental analysis, there is a notable increase in the LC due to high concentrations of RB within the pores. As with UV-Vis studies, h-MSNs seem to demonstrate superior loading capacities compared to MSNs. $\mathrm{C}_{1}$ had slightly more $\mathrm{SiO}_{2}$ content than B1, a result we thought agrees with TEM images showing increased TEOS concentration resulting in individual aggregates of silica that are not deposited onto the surface. Although this data demonstrated the successful confinement of RB within the pores, the method could not be used to reliably quantitatively the load due to data showing high standard error high; a major issue caused by high noise ratio when low energy elements are analyzed using the technique

\subsection{Conclusions}

In this Chapter, we estimated the silanol number of MSNs using TGA. We used the information to partially functionalize the surface for two purposes: 1) introduce amine onto the surface to change the surface charge and use electrostatic-assisted loading to improve 
loading capacity, and 2) spare silanols for the deposition of a silica coating. The loading capacity was significantly improved by employing a novel one-pot loading and coating procedure. Unlike uncoated nanoparticles, where the amount of load is limited to available functional groups for either covalent or electrostatic interaction, coating enables the incorporation of a large number of molecules passively. Moreover, loading by surface conjugation sometimes requires functionalization of the molecules and might cause loss of chemical activity.

These findings suggest that std-MSNs and h-MSNs could be used as a loaded and coated host for CEST-MRI. hMSNs could prove superior due to their internal void, which provided ample loading volume for dye compared to MSNs. However, despite the potential demonstrated here, optimization studies still need to be done. Measurement and optimization of the permeability rates as a function of shell thickness or porosity must ensure that suitable water kinetics exist across the coating. The actual chelate has to be experimented with to assess its chemical stability during coating, and complementary loading studies must be used. 
CHAPTER 5: A MODIFIED STÖBER METHOD USING COMMERCIALLY AVAILABLE SILICA SEEDS FOR PRODUCING LARGE, SILICA NANOPARTICLES WITH LOW POLYDISPERSITY AND THEIR SUBSEQUENT USE IN THE SYNTHESIS OF RATTLE-TYPE HOLLOW MESOPOROUS SILICA NANOPARTICLES

\begin{abstract}
Because of their ease of synthesis and well-understood chemistry, silica nanoparticles have significant potential in scientific and technological applications. Controlling the nucleation and growth phases of silica nanoparticle synthesis continues to be studied to improve the quality of silica nanoparticle colloids and powders. This study describes a low-cost and simple method for producing large $(105-356 \mathrm{~nm})$, low polydispersity silica nanoparticles from small commercially available silica seeds.
\end{abstract}

We further demonstrated the technique's versatility by creating nanoparticles of varying sizes and by adding a third layer, a mesoporous silica shell, and afterward performing a structurally selective chemical etch; we were able to synthesize rattle-type hollow mesoporous silica nanoparticles. In this synthesis, silica seed particles $(\sim 22 \mathrm{~nm}$ in diameter), water, and a catalyst $\left(\mathrm{NH}_{4} \mathrm{OH}\right)$ are pre-mixed and then added to a tetraethyl orthosilicate (TEOS)/ethanol solution. The resulting mixture is stirred overnight, resulting in the formation of large, highly uniform silica nanoparticles. Because the degree of condensation between the seed and the first lab-deposited layer varied, rattle mesoporous silica nanoparticles are possible due to differences in layer density, which we support with $\mathrm{N}_{2}$ porosimetry measurements. 


\subsection{INTRODUCTION}

Silica nanoparticles $\left(\mathrm{SiO}_{2} \mathrm{NPs}\right)$ have demonstrated controllable physical, chemical, morphological, and structural properties, which has led to their expansion in several fields, including medical (drug delivery, and optical imaging), agricultural (insecticides, growth support), industrial (increasing shelf life, catalysis, and biosensors) and environmental applications. ${ }^{157,158}$ Their use in various applications depends on particle size, polydispersity, shape, and composition, all of which can, in theory, be strictly controlled in synthesis. ${ }^{92,159}$ Because geometric features determine nanoparticle properties, nearmonodisperse particles are desirable to ensure uniform properties among individual particles in the population. ${ }^{158}$

The Stöber method remains the most widely used method of producing spherical, nonporous $\mathrm{SiO}_{2} \mathrm{NPs}$. The procedure is mainly done in an alcoholic medium. It involves the hydrolysis of alkoxysilane ( $\left.\mathrm{Si}(\mathrm{OR})_{4}\right)$, where $\mathrm{R}$ is an alkyl group) and condensation of the resultant monomers in the presence of ammonia as a (basic) catalyst. ${ }^{54}$ During hydrolysis, the alkoxysilane undergoes hydrolysis with silanol groups replacing alkoxy groups in a nucleophilic attack reaction (Equation 1). Condensation also occurs via nucleophilic attack where a silanol reacts with either a neighboring silanol or alkoxy group in a condensation reaction, giving rise to the formation of siloxane bridges holding together the silica network and the liberation of either water (Equation 2) or ethanol (Equation 3). ${ }^{160}$ A scheme overview of the formation process is presented in Figure 5.1.

$$
\mathrm{Si}(\mathrm{OR})_{4}+x \mathrm{H}_{2} \mathrm{O} \rightarrow \mathrm{Si}(\mathrm{OR})_{4-x}(\mathrm{OH})_{x}+x \mathrm{ROH}
$$




$$
\begin{aligned}
& \mathrm{Si}(\mathrm{OR})_{4-x}(\mathrm{OH})_{x}+\mathrm{Si}(\mathrm{OR})_{4-x}(\mathrm{OH})_{x} \rightarrow(\mathrm{OR})_{8-2 x}(\mathrm{Si}-\mathrm{O}-\mathrm{Si})(\mathrm{OH})_{2 x-2}+\mathrm{H}_{2} \mathrm{O} \\
& \mathrm{Si}(\mathrm{OR})_{4}+\mathrm{Si}(\mathrm{OR})_{4-x}(\mathrm{OH})_{x} \rightarrow(\mathrm{OR})_{7-x}(\mathrm{Si}-\mathrm{O}-\mathrm{Si})(\mathrm{OH})_{x-1}+\mathrm{ROH}
\end{aligned}
$$

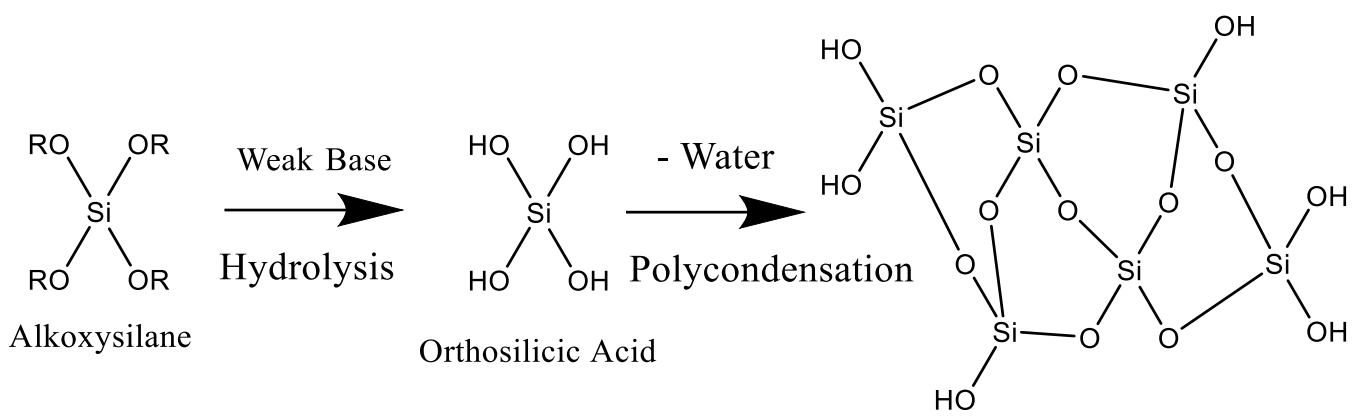

Where $\mathrm{R}$ is an alkyl group

Silica

Figure 5.1 The sol-gel method is used to create $\mathrm{SiO}_{2}$ NPs from tetraethoxysilane in aqueous ethanolic solutions. During hydrolysis, silanol groups replace alkoxy groups in a nucleophilic substitution reaction. Thus, the alkoxysilane is hydrolyzed. This is followed by a condensation reaction in which neighboring silanols react, giving off water and forming siloxane bridges that hold the silica together.

There is experimental evidence that the bottom-up approach synthesis of $\mathrm{SiO}_{2} \mathrm{NPs}$ follows the classical nucleation theory (CNT). The associated sol chemistry affects the rates of hydrolysis and condensation, which in turn affects nucleation, aggregation, and the growth of particles. ${ }^{161,162}$ Initially, negatively charged, deprotonated orthosilicic acid units resulting from the hydrolysis of alkoxysilane accumulate in the reaction medium, causing an increase in the Gibbs free energy of the system due to supersaturation. At this point, the resultant polymeric structures would have reached a size and degree of crosslinking where they become large enough to be insoluble and simultaneously form many nuclei; nucleation lowers the Gibbs free energy of the system, taking it back to (monomer) saturation. In one theory, particle growth occurs due to the primary nuclei growing by the solution's adsorption of the remaining orthosilicic acid monomers. The removal, or dilution, of these monomers ensures the monomer concentration in the solution does not reach saturation again, which avoids secondary nucleation that would give rise to size polydisperse 
particles. The second proposed growth mechanism is the aggregation growth model, where the primary particles (nuclei) aggregate into more significant masses to form larger particles.

The two theories have since been supported to both occur, with experimental and simulated studies noting the disparity between the number of initial nuclei and the final number of particles in some cases (aggregation mechanism), as well as a reduction in monomer concentrations over time. For instance, Han et al. demonstrated the in situ seeded growth nature of the Stöber process, demonstrating that the nucleation and growth processes occurred at a distinct point in time. They discovered that small $\mathrm{SiO}_{2} \mathrm{NPs}_{\text {s nucleate and grow }}$ siloxane network clusters via condensation of surrounding silanol monomers. $\mathrm{SiO}_{2}$ nanoparticles expand as previously formed silicon particles combine with newly hydrolyzed silanol monomers. They were thus able to reconcile the aggregation-only and monomer addition theories into a single consistent framework for comprehending the Stöber process through this study. ${ }^{160}$

Because the nucleation and growth phases are inextricably linked and highly dependent on physicochemical parameters, ${ }^{55,58}$ research has gone into optimizing the Stöber synthesis parameters to facilitate simultaneous nucleation and avoid secondary nucleation that leads to size heterogeneity. ${ }^{77}$ Dropwise precursor addition, techniques to promote burst (short) nucleation, and seed-mediated growth have been the primary methods explored to date for increasing $\mathrm{SiO}_{2}$ nanoparticle size homogeneity. Short nucleation times lead to the creation of initial nuclei that later grow simultaneously, and if precursor homogeneity is achieved 
in the starting, monodisperse particles should be achieved. Burst nucleation can be accomplished by causing a sudden increase in monomer concentration (orthosilicic acid), followed by a rapid decrease in monomer concentration below nucleation levels, which effectively inhibits secondary nucleation. The uniformity of the growing colloidal particles can thereafter be maintained under conditions of diffusion-controlled growth, which is accomplished in practice by maintaining low monomer concentrations, increasing the viscosity of the synthesis medium, or introducing a diffusion barrier, e.g., a polymeric monolayer. ${ }^{163}$

Another way of avoiding secondary nucleation in synthesis is controlling growth by using the seed growth technique. In a seeded synthesis method, small preformed nanoparticles act as nuclei on which growth can occur; thus, the monodispersity of the seed particles can be translated to the larger particles if the monomer concentration is kept low enough that secondary nucleation is avoided. Seeded methods have been used with reasonable success by others to prepare solid $\mathrm{SiO}_{2}$ NPs and many other types of NPs (e.g., Au, Ag). ${ }^{96,164,165}$ For many other types of NPs, including popular plasmonic gold and silver NPs, seeded methods have significantly advanced synthetic methodologies and applications by affording both predictable size and morphology control, leading to extended methods to $\mathrm{SiO}_{2}$ particles. For example, Quan et al. used the in situ addition of TEOS and applied the

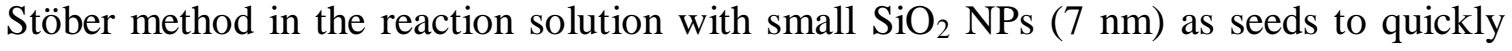
generate large $\mathrm{SiO}_{2}$ NPs $(100 \mathrm{~nm}) .{ }^{166}$ Kim et al. managed to synthesize $50 \mathrm{~nm}$ - $120 \mathrm{~nm}$ sized low polydispersity $\mathrm{SiO}_{2}$ NPs using two-phase (water/cyclohexane) synthesis 
techniques and argued that the seeded techniques by-pass nucleation which is the least controllable stage of $\mathrm{SiO}_{2} \mathrm{NPs}$ formation. ${ }^{56}$

Despite the success of seeded procedures, they are still hampered by some limitations: the poor reproducibility of the methods of making seed particles, the difficulty of surfactant removal from the seeds, strict control over physico-chemical parameters in the synthesis of larger particles, and the utilization of two-phase synthesis protocols which tend to be laborious and use-harmful organic solvents. Herein, we demonstrate a simple seeded synthesis method for the preparation of highly size uniform $\mathrm{SiO}_{2}$ nanoparticles in this size range (105 nm - $356 \mathrm{~nm}$ ), as well as rattle-type hollow mesoporous $\mathrm{SiO}_{2} \mathrm{NPs}$, derived from them based on density differences in the deposited $\mathrm{SiO}_{2}$ layers onto commercially available Ludox TM-40 silica seeds.

This product has recently been used as a reference material for DLS size and zeta potential analysis due to its high size uniformity and long-term colloidal stability. Although LudoxTM40 has been used for other applications, e.g., synthesis of nanocomposite polymer foams, transparent silica nanoparticulate films, carbon nanocapsules, and more, ${ }^{167,168}$ to the best of our knowledge, it has not been used as seed material to produce larger $\mathrm{SiO}_{2}$ nanoparticles. Ludox-TM40 is electrostatically rather than sterically stabilized (e.g., by surfactants), making it readily available for use as a seed as-received. Furthermore, we note that $\mathrm{SiO}_{2}$ NPs of larger sizes are also commercially available but relatively much costlier vs. smaller ones and that their syntheses are trademarked, limiting user access. 
The seeded synthesis process we demonstrate here is designed to fully separate nucleation and growth stages and enable wide access to large $\mathrm{SiO}_{2} \mathrm{NPs}$, using cheap reproducibly obtained seeds. We also demonstrate the use of the larger particles to prepare higher complexity, rattle-type hollow mesoporous $\mathrm{SiO}_{2} \mathrm{NPs}$ with a good translation of the size homogeneity of the seeds throughout the steps. Overall, in this chapter/paper, using our seeded Stöber $\mathrm{SiO}_{2} \mathrm{NPs}$, we synthesized rattle $\mathrm{SiO}_{2}$ NPs demonstrating for the first time that beyond structural differences (mesoporous vs. nonporous), r-hMSNs can be synthesized using differences in porosity (degree of condensation in non-porous $\mathrm{SiO}_{2}$ ).

\subsection{EXPERIMENTAL}

\subsubsection{Materials}

Tetraethoxysilane 98\% (TEOS), Ludox TM-40 (40\% ( $\left.\mathrm{w}_{\text {silica }} / \mathrm{w}_{\text {total }}\right)$ aq. colloid), and cetyltrimethylammonium bromide $(\mathrm{CTAB}, \geq 99.0 \%)$ were purchased from Sigma-Aldrich. Aqueous ammonium hydroxide $\left(\mathrm{NH}_{4} \mathrm{OH}(\mathrm{aq}), 30 \%\right.$ w/w) was purchased from Fisher. absolute $\mathrm{EtOH}$ (absolute $\mathrm{EtOH}, 200$ proof) was bought from Decon labs Inc., and electrophoretically pure $\mathrm{H}_{2} \mathrm{O}\left(\mathrm{mp} \mathrm{H}_{2} \mathrm{O}, 18 \mathrm{M} \Omega \bullet \mathrm{cm}\right.$ resistivity) was used for all synthesis protocols. Laboratory deionized water $\left(\mathrm{DI}-\mathrm{H}_{2} \mathrm{O}\right)$ and ethanol $(\mathrm{EtOH}$, reagent grade) was used for all purification (washing) steps. Experiments were performed in triplicates and repeated at least three times with similar results. For representative samples, the mean \pm standard deviation was reported.

\subsubsection{Synthesis of Nanoparticles}


Seeded Synthesis of $\mathrm{SiO}_{2}$ NPs: Large $\mathrm{SiO}_{2} \mathrm{NPs}$ (SS1) were synthesized in a facile method that combines an all-at-once (modified Stöber) procedure with a commercial silica seed (Ludox TM-40) to translate the low polydispersity of the commercially available small nanoparticles to larger $\mathrm{SiO}_{2} \mathrm{NPs}$ and more complex r-hMSNs products. In this synthesis, a solution containing $75 \mathrm{~mL}$ of absolute EtOH and $3.12 \mathrm{~mL}$ of TEOS was first prepared and stirred at a rate of $450 \mathrm{rpm}$ at room temperature for about 15 minutes. After that, a mixture of millipore $\mathrm{H}_{2} \mathrm{O}(1.29 \mathrm{~mL}), 20.4 \mu \mathrm{L}$ of Ludox TM-40 (as-received), and aqueous $\mathrm{NH}_{4} \mathrm{OH}(4 \mathrm{~mL})$ was prepared and subsequently added to the stirring ethanolic TEOS solution; the reaction proceeded at room temperature overnight. Afterward, the resulting suspension was centrifuged at $18,000 \mathrm{Fc}$ for $20 \mathrm{~min}$ and washed three times with DI water, $70 \%$ w/w aq. EtOH, and 95\% w/w aq. EtOH, respectively. The dried nanoparticle powders were then re-dispersed in $17.5 \mathrm{~mL}$ absolute $\mathrm{EtOH}$ at a $\sim 52 \mathrm{mg} / \mathrm{mL}$ concentration for storage, subsequent use, and analysis. To investigate the effects of $\mathrm{NH}_{4} \mathrm{OH}$ concentration, SS2 and SS3 were synthesized by increasing the $\mathrm{NH}_{4} \mathrm{OH}$ concentration. The volume of absolute $\mathrm{EtOH}$ was changed to keep the concentrations of the other reagents constant in these tests.

Synthesis of $\mathrm{SiO}_{2}$ rattle-type HMSNs: After the growth of larger silica NPs from the commercial seeds, a (third) mesoporous silica layer was deposited onto the large (bilayer) $\mathrm{SiO}_{2} \mathrm{NPs}$ to synthesize rattle-type hollow mesoporous silica nanoparticles (r-hMSNs). Starting with SS1 dispersed at $\sim 52 \mathrm{mg} / \mathrm{mL}$ in absolute $\mathrm{EtOH}, 3.5 \mathrm{~mL}$ of the stock $\mathrm{SiO}_{2} \mathrm{NP}$ suspension, $0.06 \mathrm{~g} \mathrm{CTAB}, 33 \mathrm{~mL}$ water, and $28 \mu \mathrm{L}$ of TEA were stirred at $600 \mathrm{rpm}$ for 
50 minutes at $80{ }^{\circ} \mathrm{C}$. Afterward, the stirring rate was increased to $1400 \mathrm{rpm}$, and $144 \mu \mathrm{L}$ of TEOS was added. The reaction was allowed to stir for 4 hours. The resultant mesoporous silica-coated Stöber NPs were centrifuged at 18,000 Fc for 20 min and washed three times with $95 \% \mathrm{EtOH}, 70 \% \mathrm{EtOH}$, and DI water, respectively. To partially remove the core and prepare rattle-type hMSNs, the mesoporous silica-coated $\mathrm{SiO}_{2}$ (trilayer) nanoparticles were resuspended in $10 \mathrm{~mL}$ of DI water through sonication until the mixture was homogenous. Meanwhile, $1.33 \mathrm{~g}$ of solid sodium carbonate was mixed with $10 \mathrm{~mL}$ of DI water at a stirring rate of $600 \mathrm{rpm}$ for 30 minutes at $50{ }^{\circ} \mathrm{C}$. The sonicated NP suspension was then added to the sodium carbonate solution, and the stirring rate was increased to $1200 \mathrm{rpm}$. The etching reaction proceeded at this rate for 10 hours, and the resultant etched particles were washed three times with DI water, $70 \%$ w/w aq. EtOH, and $95 \%$ w/w aq. EtOH, respectively.

Afterward, to free the CTAB-filled pores of the mesoporous silica shell, the resultant pellet containing the NPs was resuspended in a $100 \mathrm{~mL} \mathrm{EtOH} / \mathrm{HCl}$ solution $(9: 1 \mathrm{v} / \mathrm{v})$ and refluxed at $90{ }^{\circ} \mathrm{C}$ for 1 hour. This etching procedure was repeated until no CTAB was detectable by FT-IR spectroscopic analysis. Following four washing cycles, the clean r-hMSNs were oven-dried at $60{ }^{\circ} \mathrm{C}$ overnight. An inverse-etching procedure was also done to investigate the effect of changing the etching order (core/shell vs. shell/core). In this experiment, mesoporous silica-coated $\mathrm{SiO}_{2}$ NPs were prepared the same as above. However, the order of acid etching (to remove $\mathrm{CTAB}$ ) and sodium carbonate (to remove middle silica layer) etching steps was reversed. 


\subsubsection{Characterization}

The structural features of the synthesized nanoparticles were analyzed by TEM using Technai F20 TEM operating at $4500 \mathrm{eV}$ (CEMN, Portland State University). The microstructural composition of the $\mathrm{SiO}_{2}$ NPs was investigated by FT-IR spectroscopy (Thermo Nicolet iS10 of solid NP samples, using a diamond ATR attachment). The hydrodynamic radius was measured using a DLS particle analyzer (Horiba LB-550). $\mathrm{N}_{2}$ porosimetry isotherms were obtained at $77 \mathrm{~K}$ using a Micromeritics ASAP 2020 surface area analyzer. The samples' specific surface areas and pore size distribution were calculated using the BET and BJH methods described in the previous chapters. For calculating the PdI, which is a measurement of a nanoparticles' average homogeneity (size uniformity), Eqn 3.1 in Chapter 3 was used.

\subsection{RESULTS AND DISCUSSIONS}

\subsubsection{Synthesis of seeded Stöber nanoparticles}

The Ludox TM-40-seeded synthesis procedure presented here is based on the novel Stöber synthesis protocol. The Ludox TM-40-seeded synthesis approach disclosed in this research is based on a published dropwise method that employed $4 \mathrm{~mL}$ of TEOS and produced roughly $100 \mathrm{~nm}$ particles with a yield ( $\left.\mathrm{Y}_{\text {mass }}\right)$ of 0.9 grams. ${ }^{129}$ We calculated the number of particles in the $0.9 \mathrm{~g}$ sample $\left(\mathrm{N}_{\mathrm{ss} 1}\right)$ (= number of nucleation sites) using Eqn 5.4:

$$
N_{S S 1}=\left[\frac{Y_{\text {mass }}}{\rho_{S i O_{2}}}\right] * \frac{1}{V_{S S 1}}
$$


Where $\rho_{\mathrm{SiO}_{2}}$ is the density of $\mathrm{SiO}_{2}$, i.e., $2.2 \mathrm{~g} / \mathrm{cm}^{3}, \mathrm{~V}_{\mathrm{ss} 1}$ is the volume of $1 \mathrm{SiO}_{2} \mathrm{NP}$ (SS1) Assumption: All nucleation centers gave rise to spherical $100 \mathrm{~nm}$ NPs

We then calculated the amount of TEOS required to make approximately the same size of the particles starting the Ludox TM-40 seeds by using Eqn 4.2 in Chapter 4. The nanoparticles synthesized using the reagent amounts listed above are highly spherical and have narrow size distribution, as evidenced by the small standard presented in Table 6.1. The TEM size distributions for synthesis sample variants SS1, SS2, and SS3 are summarized in Table 5.1, and Figure 5.2 shows the representative TEM images.

Table 5.1 Experimental conditions and results. A summary of the reagents and concentrations used in a study of the effects of the catalyst concentration on the synthesis of large $\mathrm{SiO}_{2} \mathrm{NPs}$. The TEM size of the seed nanoparticles was 22.2 (4.5)

\begin{tabular}{llllll} 
Sample & $\begin{array}{l}\text { Absolute } \\
\text { EtOH }(\mathbf{m l})\end{array}$ & $\begin{array}{l}\text { TEOS } \\
(\mathbf{m M})\end{array}$ & $\begin{array}{l}\mathbf{H}_{2} \mathbf{O} \\
(\mathbf{m M})\end{array}$ & $\begin{array}{l}\mathbf{N H}_{4} \mathbf{O H} \\
(\mathbf{m M})\end{array}$ & $\begin{array}{l}\text { Average } \\
\text { TEM } \\
\text { size(nm) }\end{array}$ \\
\hline SS1 & 75 & 176.2 & 858.2 & 704 & $105.2 \pm 5.9$ \\
SS2 & 71 & 176.2 & 858.2 & 1409 & $152.8 \pm 6.1$ \\
SS3 & 67 & 176.2 & 858.2 & 2115 & $356.6 \pm 9.9$
\end{tabular}

The size of the seed nanoparticles was found to be $22.2 \mathrm{~nm}$ (Figure 5.2A), which is very close to the manufacturer's reported value of $22 \mathrm{~nm}$. An SD of 4.5 indicates a narrow size distribution, which may justify their use as a calibration standard in size measuring instruments. Using a low-cost form of monodisperse colloidal silica as seed enabled subsequent growth on a lab-synthesized layer via abundant surface hydroxyls, effectively translating the narrow size distribution to the larger $\mathrm{SiO}_{2} \mathrm{NPs}$; SS1, SS2, and SS3 (5.2 B- 
D). TEM images show that SS1, SS2, and SS3 have uniform size distributions of $105.2 \pm 5.9 \mathrm{~nm}, 152.8 \pm 6.1 \mathrm{~nm}$, and $356.6 \pm 9.9 \mathrm{~nm}$, respectively. These results support previously reported results that $\mathrm{NH}_{4} \mathrm{OH}$ concentration has a positive impact on particle size growth. The observed trend is due to an increase in hydrolysis and condensation rates. ${ }^{56,135}$ For comparison, Figure 5.2 E shows a TEM image of a representative sample synthesized using a non-seeded synthesis method under similar concentration conditions to those of SS1 in Table 5.1. Some other observations from the TEM images are that sample SS3, with the highest $\mathrm{NH} 4 \mathrm{OH}$ concentration, had some small clusters (debris) intermittently observed on the grid (Figure 5.2 D), which we suspected was due to the presence of residual
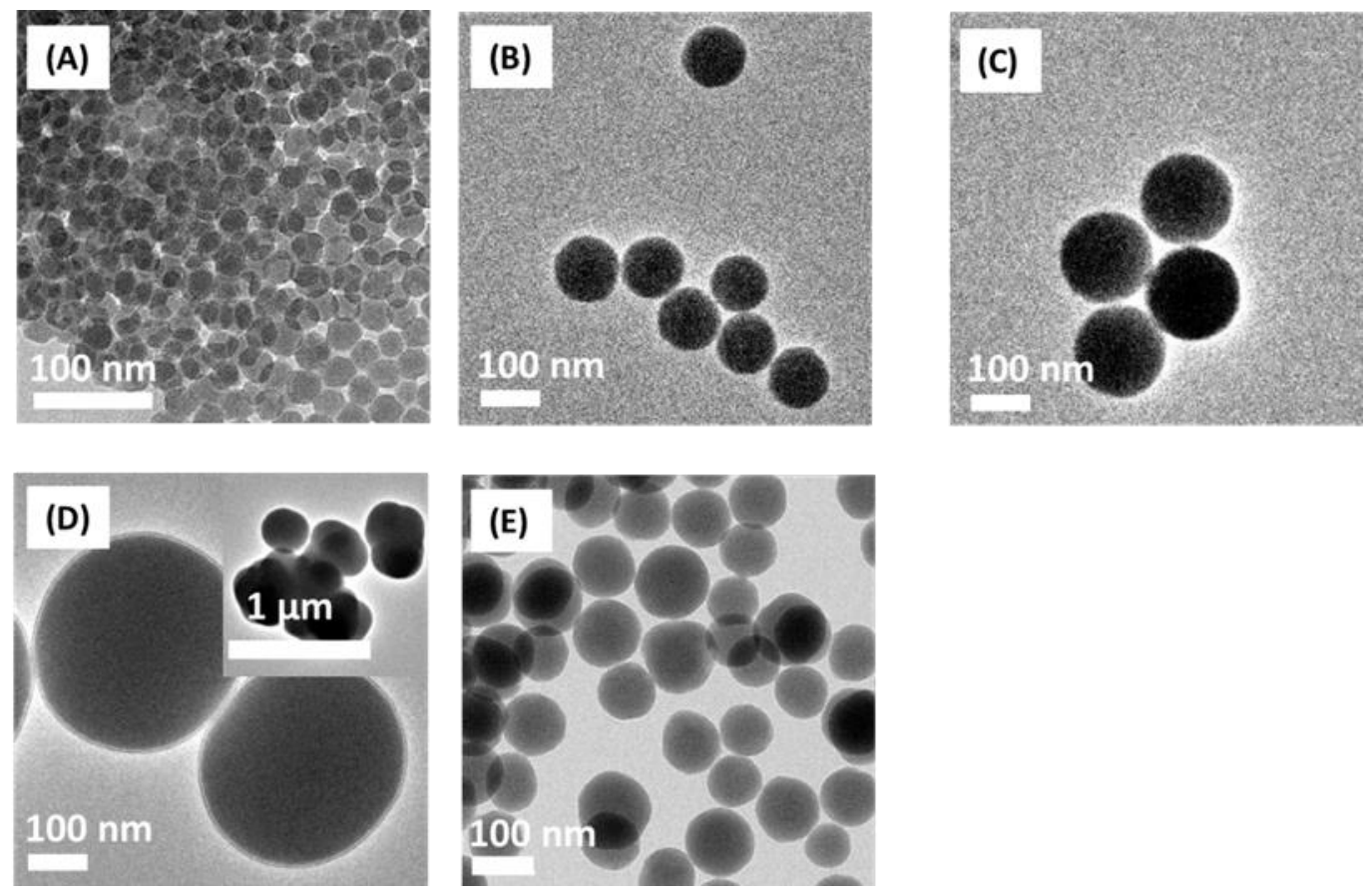

Figure 5.2 Representative TEM images for Ludox-TM40 (A), larger $\mathrm{SiO}_{2} \mathrm{NP}$ samples SS1 (B), SS2 (C), and SS3 (D), and a comparative TEM image from a non-seeded synthesis $(E)$ under otherwise similar synthesis conditions 
water after washing during the TEM sample preparation and oven heating step (this has previously been associated with aggregation during sample prep168). These particles had a mean size of $93.2 \pm 23.5 \mathrm{~nm}$, indicating a much broader size distribution than those observed for the seeded NPs SS1, SS2, and SS3.

DLS data further supported the TEM images revealing that all samples (SS1, SS2, and

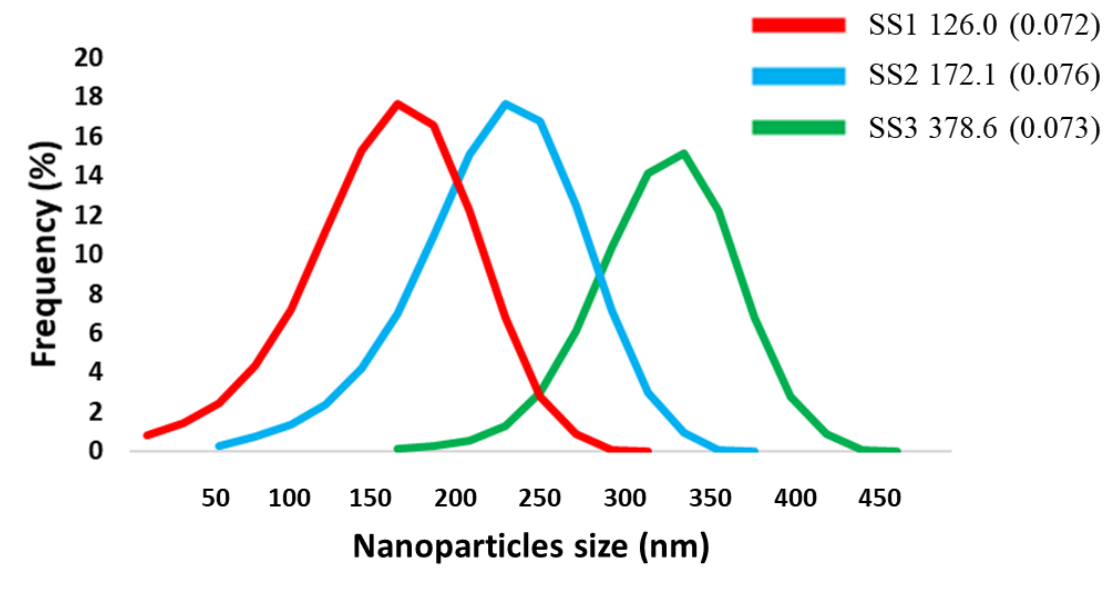

Figure 5.3 DLS distributions for SS1, SS2, and SS3 with modal sizes and PdIs. The increase in particle size as ammonium hydroxide concentrations rise is noted

SS3) synthesized using the seeded method are highly are monodisperse (Figure 5.3). The data show that the hydrodynamic diameters of the synthesized NPs SS1, SS2, and SS3 are $126.0 \mathrm{~nm}, 172.1 \mathrm{~nm}$, and $378.9 \mathrm{~nm}$, respectively, with corresponding PdIs of 0.072, 0.076, and 0.073. Despite some studies reporting an increase in PdI as particle size increases (due to an increase in $\mathrm{NH}_{4} \mathrm{OH}$ concentration), ${ }^{138,169}$ our particles did not show this apparent trend. The difference in size measurements between DLS and TEM is expected because DLS distributions are based on the light intensity of the sample nanoparticles and measure 
the hydrodynamic diameters. In contrast, TEM distributions are based on light transmission and measure the NP cores only. ${ }^{170}$

Silica formation in the Stöber synthesis is a two-step process that begins with the hydrolysis of TEOS and ends with the polycondensation of its monomers (silicic acid) to generate silica networks. Following the addition of the seed, catalyst, and water mixture to an already homogeneous TEOS solution, the hydrolysis of the TEOS results in the monomer accumulation in the starting solution, which eventually reaches saturation condensation of silica on the seed surface occurs without secondary nucleation. By using the pre-formed seeds to bypass the nucleation step, the possibility of heterogeneous nucleation is avoided or reduced; it is well known that heterogeneous nucleation causes polydisperse nanoparticle formation (e.g., the La Mer mechanism of NP formation).

\subsubsection{Synthesis of r-hMSNs}

Rattle-type $\mathrm{SiO}_{2}$ NPs have been gaining popularity for imaging, confined-space catalysis, thermal and electrical insulation, and drug delivery due to their unique structural and morphological properties, such as low density, large surface area, excellent small molecule loading capacity, and high permeability. ${ }^{139,171,172}$ Compared to hollow $\mathrm{SiO}_{2}$ nanoparticles, which have an empty void inside, rattle-type $\mathrm{SiO}_{2}$ nanoparticles possess a metal core or yolk-like particles (such as $\mathrm{Au}, \mathrm{Fe}_{2} \mathrm{O}_{3}$, and $\mathrm{Fe}_{3} \mathrm{O}_{4}$ ) within the interior; thus, they have a core-void-shell structure. The core-void-shell configuration gives rattle-type $\mathrm{SiO}_{2} \mathrm{NPs}$ extra surface area and provides the opportunity to functionalize the inner surface(s) differently from the outer surface. ${ }^{60,173}$ A mesoporous shell, for example, can allow the 
passage of small reactants $\mathrm{HAuCl}_{4}(\mathrm{aq})$ into a selectively amine-functionalized silica rattle, which, when heated, results in the synthesis of Au nanoparticles within the rattle structure, and thus the overall nanostructure acts as a nanoreactor. ${ }^{173}$ Further, Zhang et al. demonstrated that silica rattle-silica shell nanostructures exhibit a two-step drug elution profile and argued that such a property could be applied in programmed sequential drug delivery in cases where two or more drugs are loaded. ${ }^{78}$

Prior syntheses of rattle-type $\mathrm{SiO}_{2}$ NPs have mainly been achieved through compositionalor structural-selective etching of the middle sacrificial layer or by calcination of organic middle layers. The middle layer in compositional-selective etching is made up of organic/inorganic silica hybrids. The organic part provides etching selectivity due to functional groups specifically targeted for dissolution by a given etchant. Tang et al. demonstrated one such method by synthesizing silica rattle-silica shell nanoparticles with $\mathrm{N}$-[3-(trimethoxysilyl)propyl] ethylenediamine in the sacrificial layer, etching it out with hydrofluoric acid, leaving the condensed silica core and shell intact. ${ }^{171}$ In another work, selective compositional etching was applied to synthesize different variations of rattle-type $\mathrm{SiO}_{2} \mathrm{NPs}^{49,174}$

Alternatively, structural-based etching methods have been developed to take advantage of the fact that morphological differences between different silicas (e.g., mesoporous vs. solid) exhibit selectivity to etching (e.g., different rates due to different degrees of condensation), and this reactivity difference has been used to synthesize rattle-type silica core-silica shell NPs previously. ${ }^{175}$ We synthesized rattle $\mathrm{SiO}_{2}$ NPs using our seeded Stöber 
$\mathrm{SiO}_{2}$ NPs, demonstrating for the first time that $\mathrm{r}-\mathrm{hMSN}$ can be synthesized using porosity differences rather than structural differences (mesoporous $v s$. nonporous) (degree of condensation in non-porous $\mathrm{SiO}_{2}$ ).

Using a modified Stöber technique and CTAB as the pore-forming agent, the abovementioned monodisperse $\mathrm{SiO}_{2}$ NPs were coated with a mesoporous silica shell. TEM examination revealed the successful deposition of a silica shell with a thickness of about $12 \mathrm{~nm}$ (Figure 5.4A). CTAB acts as the template for inducing pores into the silica network
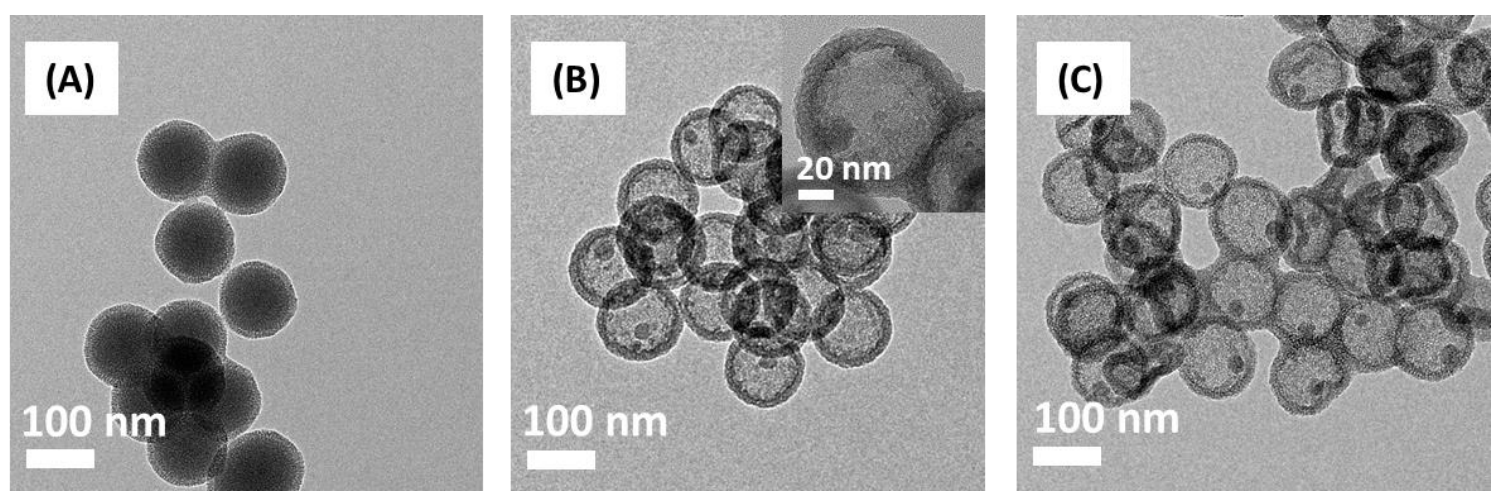

Figure 5.4 TEM images (A) showing a thin mesoporous shell coated-SS1 with CTAB still in pores (128.4 \pm 4.5$)$, (B) coreetched hybrid nanoparticles (124.0 \pm 5.3$)$ showing small rattle $(\sim 24 \mathrm{~nm})$ with a magnified image in the insert (C) completely $r$-hMSNs after etching

and forms spherical micelles in the aqueous media. The outer hydrophilic surface of CTAB with the cationic head groups interacts with hydrolyzed TEOS anions (via electrostatic attractions), creating small nucleation centers. Polycondensation forms an amorphous silica layer surrounding the micelles, creating a $\mathrm{CTAB} / \mathrm{SiO}_{2}$ hybrid layer on the core $\mathrm{SS} 1$ particles. The successful deposition is also said to have been aided by silanol on the SS1 surface, enabling the silicates to be identified interfacially, encouraging surface condensation, and inhibiting the formation of other nuclei. ${ }^{77}$ Additionally, FTIR analysis 
(unreported data) of the unetched r-hMSNs has peaks that are attributed to CTAB: $2920 \mathrm{~cm}^{-1}$, and $2850 \mathrm{~cm}^{-1}$, that are caused by the asymmetric and symmetric vibrations of the $\mathrm{CH}_{2}$ groups, respectively, ${ }^{98,99}$ indicating the presence of CTAB in the mesoporous silica shell and the effectiveness of the shell deposition.

The SS1 with the CTAB/SiO 2 hybrid layer was subsequently etched with $\mathrm{Na}_{2} \mathrm{CO}_{3}$, which selectively etched out the core leaving the original Ludox-TM40 intact (see Figure 5.4B core-etched NP with a $\sim 24 \mathrm{~nm}$ (insert) rattling). The core was selectively etched out over the shell due to its lower condensation degree, which affords it a more open structure and makes it more vulnerable to the $\mathrm{NaCO}_{3}$ than the shell with a greater condensation degree. Moreover, core etching allows etchant species $\left(\mathrm{OH}^{-}\right)$to access the core while tightly retaining the silica network in the shell. ${ }^{62}$ The rattling suggests different selective mechanisms between lab-generated and commercial silica layers beyond structural variations, which could be because of differences in porosity.

Finally, CTAB was removed from the core etched nanoparticles by refluxing them with an ethanol/HCl mixture giving rise to $\mathrm{r}-\mathrm{hMSNs}$ (Figure $5.4 \mathrm{C}$ ). The technique works by interfering with the electrostatic interaction between the anionic silicates and the cationic heads of the surfactant using $\mathrm{H}^{+}$ions from the acid. $\mathrm{H}^{+}$ions percolate through the entire silica network electrostatically exchanging with $\mathrm{CTA}^{+}$due to the significantly weaker contact between $\mathrm{CTA}^{+}$and silicates at the interface. ${ }^{140}$ FTIR was once again used to confirm the effectiveness of the etching and the disappearance of the CTAB peaks indicative of a successful etching procedure. 
To investigate porosity, $\mathrm{N}_{2}$ porosimetry was performed on Ludox-TM40, SS1, and rhMSNs (Figure 5.5 A-C). Ludox-TM40 and SS1 (Figure 5.5 A and B) have isotherms typical of Type II materials with micropores. However, the porosity of the two samples varies. Ludox -TM40 has a $0.131 \mathrm{~cm}^{3} / \mathrm{g}$ total pore volume versus SS1's $0.276 \mathrm{~cm}^{3} / \mathrm{g}$. Given the smaller size of Ludox-TM40 compared to SS1, a larger surface area to volume ratio would have been predicted; however, the two almost have the same $\mathrm{S}_{\mathrm{BET}}\left(74.1 \pm 0.81 \mathrm{~m}^{2} / \mathrm{g}\right.$ vs. $78.0 \pm 1.94 \mathrm{~m}^{2} / \mathrm{g}$; Ludox-TM40, $\mathrm{SS} 1$, respectively). We deduced that the difference in porosity between the Ludox-TM40 and the lab-synthesized solid silica layer led to the observed selective etching of the lab synthesized layer over the Ludox TM-40 templates.

(A)

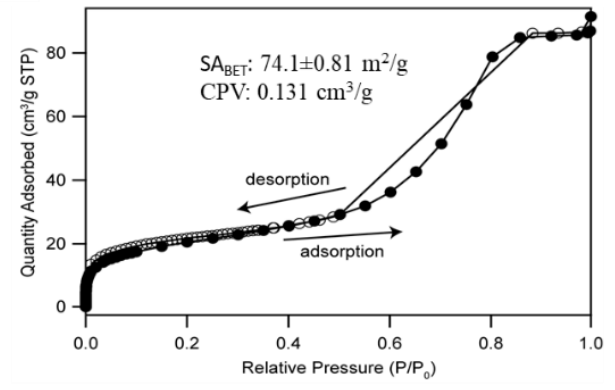

(C)

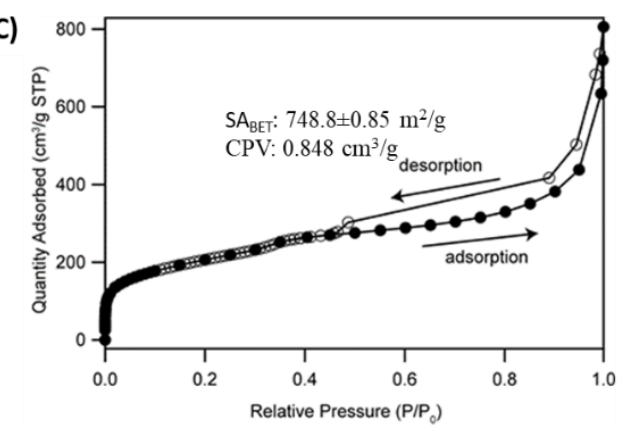

(B)

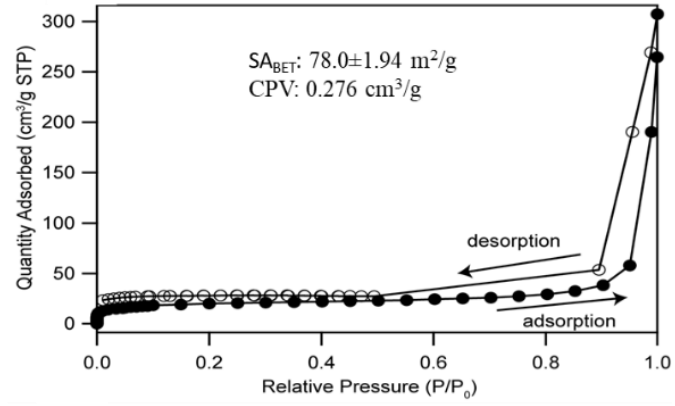

(D)

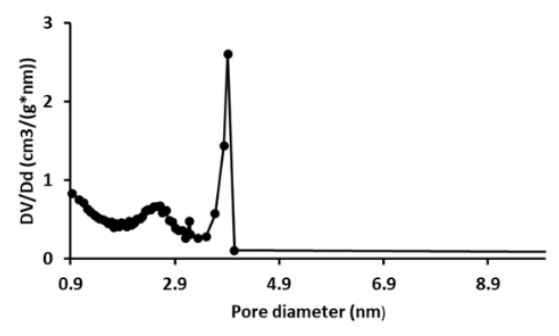

Figure 5.5 Nitrogen porosimetry studies showing surface area and cumulative pore volumes for (A) LudoxTM40, (B) Seeded SS1 and (C) Complete r-hMSNs and (D) Pore size distribution as determined by the JBH method)

The isotherm for r-hMSNs shows the typical Type-IV curve at relatively high pressure 
(Figure 6.4C) and the existence of a distinct hysteresis loop indicating mesopores' presence within the structure. A pronounced hysteresis loop indicates the presence of mesopores within the structure, which is caused by capillary condensation of the gas at high relative pressures. The resultant $\mathrm{r}-\mathrm{hMSNs}$ have a high $\mathrm{S}_{\mathrm{BET}}$ of $748.8 \pm 0.85 \mathrm{~m}^{2} / \mathrm{g}$ and a cumulative pore volume of $0.848 \mathrm{~cm}^{3} / \mathrm{g}$, showing that they are highly porous. The pore size distribution peaked at about $3.8 \mathrm{~nm}$ (Figure 6.4D), showing that the pores are large enough to allow small molecules and could expand the application of such materials.

\subsection{Conclusions}

An easy, non-laborious, inexpensive, and highly reproducible procedure for $\mathrm{SiO}_{2} \mathrm{NPs}$ fabrication was presented. Using a low-cost monodisperse commercial standard as a seed ensures that high monodispersity was maintained in the larger nanoparticles and is essential for reproducibility. The method's versatility was further demonstrated when alteration of the ammonium hydroxide concentration showed direct control over the size of the resulting nanoparticles. Such a production method can be necessary for synthesizing research-use nanoparticles as variations exist between current batches, making it hard to optimize and move research from benchtops to applications. The resultant nanoparticles were then used for making r-hMSNs by making use of porosity differences between the seed and regenerated silica layer. This was the first demonstration that beyond structural differences, porosity could be used for selective etching. 


\section{CHAPTER 6: CONCLUSIONS}

The overarching hypothesis was that MSNs could become biologically stable hosts for in vivo CEST-MRI by optimizing surface chemistry, volume, and encapsulation. This thesis project aimed to reliably create synthetic access to two MSNs with different internal morphologies, compare loading capacities and assess if a solid $\mathrm{SiO}_{2}$ coating could effectively encapsulate a negatively charged surrogate dye within the interiors of the MSNs. Achieving these goals would increase our understanding of how morphological and surface chemical features can be optimized to achieve high internal payload concentration and aid in identifying a lead MSN host candidate to optimize the water exchange rate between interior and exterior compartments in future work.

\subsection{MESOPOROUS SILICA NANOPARTICLES}

Batch-to-batch variations in MSN synthesis techniques limit prospective application studies due to inconsistencies in physical-chemical properties. The production and characterization of three types of mesoporous silica nanoparticles were discussed in this Chapter.

The approach described here was adapted from the literature, employing CTAB instead of CTAC as the templating surfactant. Discrepancies in following research protocols have been documented from lab to lab; hence the modified literature method was pursued to be adaptable in our lab. Swelling agents were utilized to expand the pore size; decane was already used in the synthesis, and DIPB had not been tried this approach. We speculated that these hydrophobic molecules got trapped within CTAB micelles, bulking the template 
size, thus making the pore size bigger. We chose a specific stirring speed since it has been identified as a significant factor in batch-to-batch changes in the literature. The synthesis method for the std-MSNs yielded extremely repeatable nanoparticles sizes, unlike the decMSNs and DIPB-MSNs.

The SD value of the std-MSNs was lower than dec-MSNs and DIPB-MSNs, which indicated that std-MSNs had a narrow size distribution. The addition of swelling agents led to three changes: 1) a size reduction, which deviates from the literature's tendencies, 2) a broadening of the size distribution, and 3) a loss in reproducibility. These findings were attributed to a decrease in the $\mathrm{CMC}$, which resulted in an increased number of nucleation sites and a smaller size, and an uneven distribution of swelling agents within the micelles. The applicability of swelling agents should involve optimizing starting reagent concentrations and conditions under which the experiment is being done.

The standard-MSNs were around $80 \mathrm{~nm}$ in overall size, which allowed for the deposition of a silica layer in Chapter 4 without exceeding the size range of nanoparticles for medical application objectives $(100 \mathrm{~nm}-200 \mathrm{~nm})$. Their $2,7 \mathrm{~nm}$ pore diameters are large enough to accommodate a significant quantity of chelate and water molecules, resulting in improved sensitivity as long as the two water pools stay separated (a role we anticipated the silica coating would be effective to perform). Together with the synthetic robustness and great reproducibility in shape and size (distribution) over DIPB-MSNs and decane-MSNs, these features make std-MSNs an excellent option for following loading and coating tests. Thus, std-MSN were selected based on their size, size homogeneity, and better synthetic 
reproducibility to move on to the subsequent functionalization, loading, and assessment stage in Chapter 4.

\subsection{HOLLOW MESOPOROUS SILICA NANOPARTICLES}

In addition to having the physical and chemical properties of MSNs, hMSNs include an interior void that could render them superior loading capacity compared to std-MSNs. We reasoned that generating hMSNs with control over the void size and shell thickness would allow us to tailor the two structural factors (concentration of internalized paramagnetic agent and the water exchange rate) independently for the best CEST-MRI contrast production. The vast void can enclose a large payload, resulting in many protons exchanging, and the shell thickness and porosity may allow fine-tuning of the water exchange rate. We can adjust the number of water molecules contained by varying the core diameters of these nanoparticles. Because of these characteristics, hMSNs appear to be a good option for CEST-MRI agents.

Although MSNs have been investigated, hMSNs have yet to be investigated in this application; this Chapter explains how hMSNs were synthesized using solid $\mathrm{SiO}_{2}$ core templates. To solve the reproducibility issue with dropwise addition methods, we developed a delayed-addition method that, when compared to an all-at-once addition method, significantly improved solid $\mathrm{SiO}_{2}$ monodispersity. This modified method resulted in higher TEOS uniformity within the starting solution before introducing the water and catalyst, making it the preferred mode for producing solid $\mathrm{SiO}_{2}$ NPs with small size 
distributions. By varying the catalyst concentration, we also made three sizes of solid $\mathrm{SiO}_{2}$ nanoparticles, an essential experimental result in designing different sized hMSNs.

We used the synthesized solid $\mathrm{SiO}_{2}$ (approximate size of $92 \mathrm{~nm}$ ) to make hMSNs by creating a mesoporous silica shell first, then performing a structural-selective etching step to remove the core template, following a literature-based method with minor modifications. The subsequent deposition of a mesoporous silica shell was performed using a method described in the literature with minor modifications. Its success was confirmed using TEM and FTIR analysis methods. The role of CTAB in protecting the mesoporous shell during core etching was established herein, and the results presented in this thesis suggest that CTAB may facilitate directional etching, in addition to providing shell protection against etching.

The hMSNs synthesized for preliminary studies had an overall diameter of approximately $135 \mathrm{~nm}$, which was enabled by using $92 \mathrm{~nm}$ solid silica nanoparticle templates. The chosen core size enabled deposition of the mesoporous shell and coating silica layer described in Chapter 4 without exceeding the size range of nanoparticles suitable for medical application objectives $(100 \mathrm{~nm}-200 \mathrm{~nm})$. Their 2,7 nm pore diameters are large enough to accommodate and allow the passage of a significant amount of chelate and water molecules into the void, which may result in increased sensitivity as long as the two water pools remain distinct (a role we anticipated the silica coating would be effective to perform). The robustness and reproducibility of the synthetic method used in this thesis enable the study of optimized experiments without batch-to-batch variation, paving the way for the loading 
and coating tests performed in Chapter 4.

\subsection{SURFACE FUNCTIONALIZATION, LOADING, AND COATING OF MSNS}

Using mesoporous silica nanoparticles, we improved the loading capacity of RB, which is a negatively charged dye. The TGA allowed us to estimate the silanol number, and we used that information to partially functionalize the surface of MSNs to accomplish two goals; 1 ) introduce amines (ammonium groups) onto the surface in order to change the surface charge and utilize the attractive electrostatic forces between the silica surface and the dye to increase the loading capacity and 2) to leave a fraction of the silanols on the surface so that condensation can be initiated in order to deposit a silica coating.

We devised a novel one-pot loading and coating procedure that significantly increased loading capacity compared to uncoated control samples (both std-MSNs and hMSNs showed a loading capacity of more than $50 \%$ compared to uncoated samples). In comparison to uncoated nanoparticles, where the number of molecules that can be included passively within the interior volume is limited by the number of functional groups accessible for covalent or electrostatic contact, the approach enables the inclusion of a large number of molecules passively within the interior volume. Additionally, the novel one-pot loading/coating process eliminates the requirement to functionalize the small molecule payload in order for it to connect with the nanoparticle host. This step can result in the load losing its functionality. The hMSNs also demonstrated superior loading than std-MSNs (71 $\%$ vs. $58 \%$ respectively), presumably due to their internal void. These findings suggest 
that MSNs could be exploited in the future as a loaded and coated host for CEST-MRI investigations. hMSNs have the potential to surpass MSNs and other forms of nanostructured CEST-MR agents conceptually due to their increased internal void volume, which gives greater residence space for the internalized contrast agent.

\subsection{A MODIFIED STÖBER METHOD USING COMMERCIALLY AVAILABLE SILICA SEEDS FOR PRODUCING LARGE SILICA NANOPARTICLES WITH LOW SIZE DISPERSITY AND THEIR SUBSEQUENT USE IN THE SYNTHESIS OF RATTLE-TYPE HOLLOW MESOPOROUS SILICA NANOPARTICLES}

Variations between existing batches make it difficult to optimize and shift research from benchtops to applications. Therefore, there is a need for synthesizing research-use nanoparticles with narrow size distribution and great reproducibility. Using a simple, nonlaborious, low-cost, and highly repeatable process, we fabricated low-size dispersity $\mathrm{SiO}_{2}$ NPs. Using a low-cost monodisperse commercial standard as a seed assures high monodispersity in the bigger nanoparticles and is critical for reproducibility. In this seeded synthesis approach, the commercial premade nanoparticles act as nuclei on which growth can proceed (via polycondensation), essentially bypassing nucleation, a critical and extremely sensitive stage for size dispersity control. As a result, the seed particles' monodispersity was carried over to the larger particles, and the optimized TEOS quantities kept monomer concentrations low enough to prevent subsequent nucleation.

The method's adaptability was further proved when the size of the resultant nanoparticles was directly controlled by changing the ammonium hydroxide concentration. The nanoparticles were then employed to make r-hMSNs by exploiting porosity differences 
between the seed and the lab-generated silica layer. $\mathrm{N}_{2}$ porosimetry measurements indicated differences in porosity between the lab-generated layer (more porous) and the seed nanoparticles (less porous), demonstrating for the first time that porosity may be employed for selective etching beyond structural differences. The seeded approach may be a viable future strategy for producing hollow nanoparticles with high repeatability that can be loaded with small CEST molecules. Additionally, the approach may enable selective functionalization of the interior and more precise loading of charged molecules into the void. Additionally, the seed can be functionalized and conjugated to a catalyst, allowing for using such particles as nanoreactors when the middle layer is selectively etched away.

Although MSNs have been investigated in CEST-MRI, the potential for hMSNs has not been investigated. Both std-MSNs and hMSNs were synthesized reproducibly with low particle and pore size dispersity. The latter was made possible by developing a delayedaddition method to synthesize the template solid silica nanoparticles. The partial amine functionalization of the MSNs' surface enabled attractive electrostatic forces during loading and facilitated the deposition of a silica coating. hMSNs demonstrated a greater capacity for loading the surrogate dye and may be more sensitive than standard-MSNs. The robust synthetic pathways increased loading capacity, and efficient encapsulation method demonstrated in this work establish a solid foundation for future experiments.

While the potential demonstrated in this work is significant, optimization studies and examinations must be conducted and examined to fully realize it. Although the silica coating was effective at containing the dye within, it is necessary to measure and optimize 
permeability rates as a function of shell thickness and porosity to fine-tune the water kinetics to the desired regime. After optimizing the water kinetics, the actual chelate would need to be loaded and its functionality assessed under post-coating conditions. The performance of such optimized systems 


\section{REFERENCES}

(1) Li, X.; Zhang, X.-N.; Li, X.-D.; Chang, J. Multimodality Imaging in Nanomedicine and Nanotheranostics. Cancer Biol. Med. 2016, 13 (3), 339-348.

(2) Caravan, P.; Ellison, J. J.; McMurry, T. J.; Lauffer, R. B. Gadolinium(III) Chelates as MRI Contrast Agents: Structure, Dynamics, and Applications. Chem. Rev. 1999, 99 (9), 2293-2352.

(3) Chen, Z.-Y.; Wang, Y.-X.; Lin, Y.; Zhang, J.-S.; Yang, F.; Zhou, Q.-L.; Liao, Y.Y. Advance of Molecular Imaging Technology and Targeted Imaging Agent in Imaging and Therapy. Biomed Res. Int. 2014, 819324.

(4) Soesbe, T. C.; Merritt, M. E.; Green, K. N.; Rojas-Quijano, F. A.; Sherry, A. D. T2 Exchange Agents: A New Class of Paramagnetic MRI Contrast Agent That Shortens Water $\mathrm{T}_{2}$ by Chemical Exchange Rather than Relaxation. Magn. Reson. Med. 2011, 66 (6), 1697-1703.

(5) De Leõn-Rodríguez, L. M.; Martins, A. F.; Pinho, M. C.; Rofsky, N. M.; Sherry, A. D. Basic MR Relaxation Mechanisms and Contrast Agent Design. J. Magn. Reson. 2015, 42 (3), 545-565.

(6) Gambhir, S. S. Just What Is Molecular Imaging. MI Gatew. 2007, 1-8.

(7) James, M. L.; Gambhir, S. S. A. Molecular Imaging Primer: Modalities, Imaging Agents, and Applications. Physiol. Rev. 2012, 92 (2), 897-965.

(8) Estelrich, J.; Sánchez-Martín, M. J.; Busquets, M. A. Nanoparticles in Magnetic Resonance Imaging: From Simple to Dual Contrast Agents. Int. J. Nanomedicine 2015, 10, 1727-1741.

(9) Evbuomwan, O. M.; Merritt, M. E.; Kiefer, G. E.; Dean Sherry, A. NanoparticleBased PARACEST Agents: The Quenching Effect of Silica Nanoparticles on the CEST Signal from Surface-Conjugated Chelates. Contrast Media Mol. Imaging 2012, 7 (1), 19-25.

(10) Sherry, A. D.; Woods, M. Chemical Exchange Saturation Transfer Contrast Agents for Magnetic Resonance Imaging. Annu. Rev. Biomed. Eng. 2008, 10 (1), 391-411.

(11) Kogan, F.; Hariharan, H.; Reddy, R. Chemical Exchange Saturation Transfer (CEST) Imaging: Description of Technique and Potential Clinical Applications. Curr. Radiol. Rep. 2013, 1 (2), 102-114.

(12) Song, B.; Wu, Y.; Yu, M.; Zhao, P.; Zhou, C.; Kiefer, G. E.; Sherry, A. D. A Europium(Iii)-Based PARACEST Agent for Sensing Singlet Oxygen by MRI. Dalt. Trans. 2013, 42 (22), 8066-8069. 
(13) Wu, B.; Warnock, G.; Zaiss, M.; Lin, C.; Chen, M.; Zhou, Z.; Mu, L.; Nanz, D.; Tuura, R.; Delso, G. An Overview of CEST MRI for Non-MR Physicists. EJNMMI Phys. 2016, 3 (1).

(14) Evbuomwan, O. M.; Merritt, M. E.; Kiefer, G. E.; Dean Sherry, A. NanoparticleBased PARACEST Agents: The Quenching Effect of Silica Nanoparticles on the CEST Signal from Surface-Conjugated Chelates. Contrast Media Mol. Imaging 2012, $7(1), 19-25$.

(15) Soesbe, T. C.; Yunkou, W.; Sherry, D. Advantages of paramagnetic CEST complexes having slow-to-intermediate water exchange properties as responsive MRI agents. NMR Biomed. 2014, 26 (7), 829-838.

(16) Pikkemaat, J. A.; Wegh, R. T.; Lamerichs, R.; van de Molengraaf, R. A.; Langereis, S.; Burdinski, D.; Raymond, A. Y. F.; Janssen, H. M.; de Waal, B. F. M.; Willard, N. P.; Meijer, E. W.; Grüll, H. Dendritic PARACEST Contrast Agents for Magnetic Resonance Imaging. Contrast Media Mol. Imaging 2007, 2 (5), 229-239.

(17) Nam, J.; Won, N.; Bang, J.; Jin, H.; Park, J.; Jung, S.; Jung, S.; Park, Y.; Kim, S. Surface Engineering of Inorganic Nanoparticles for Imaging and Therapy. Adv. Drug Deliv. Rev. 2013, 65 (5), 622-648.

(18) Kimmich, R.; Fatkullin, N. Polymer Chain Dynamics and NMR. Adv. Polym. Sci. 2004, 170, 1-113.

(19) Soesbe, T. C.; Wu, Y.; Dean Sherry, A. Advantages of Paramagnetic Chemical Exchange Saturation Transfer (CEST) Complexes Having Slow to Intermediate Water Exchange Properties as Responsive MRI Agents. NMR Biomed. 2013, 26 (7), 829-838.

(20) Tsai, Y.-L. S.; Dietz, A.; Oppelt, N.; Kuenzer, C. Remote Sensing Remote Sensing of Snow Cover Using Spaceborne SAR: A Review. Remote Sens. 2019, 11, 1456.

(21) Wang, Y.-F.; Liu, L.; Xue, X.; Liang, X.-J. Nanoparticle-Based Drug Delivery Systems: What Can They Really Do in Vivo? F1000Research 2017, 6 (5), 681.

(22) Wang, L.; Hu, C.; Shao, L. The Antimicrobial Activity of Nanoparticles: Present Situation and Prospects for the Future. Int. J. Nanomedicine 2017, 12, 1227-1249.

(23) Chen, Y. Design, Synthesis, Multifunctionalization and Biomedical Applications of Multifunctional Mesoporous Silica-Based Drug Delivery Nanosystems. Springer Theses 2016, 31-47.

(24) Aime, S.; Castelli, D. D.; Terreno, E. Highly Sensitive MRI Chemical Exchange Saturation Transfer Agents Using Liposomes. Angewandte Chemie Inter. 2005, 44, $5513-5515$.

(25) Ma, X.; Zhao, Y. Biomedical Applications of Supramolecular Systems Based on 
Host-Guest Interactions. Chem Rev. 2014, 115 (15), 7794-839.

(26) Aime, S.; Castelli, D. D.; Terreno, E. Polypeptide Imaging Agents Supramolecular Adducts between Poly-1-Arginine and [Tm III Dotp]: A Route to SensitivityEnhanced Magnetic Resonance Imaging-Chemical Exchange Saturation Transfer Agents. Angewandte Chemie. 2003, 115, 4665-4667.

(27) Geninatti, S.; Ae, C.; Barge, A.; Battistini, E.; Claudia, A. E.; Ae, C.; Coluccia, S.; Longo, D.; Valentina, A. E.; Ae, M.; Tarone, G.; Aime, S. Magnetic Resonance Imaging Visualization of Targeted Cells by the Internalization of Supramolecular Adducts Formed between Avidin and Biotinylated $\mathrm{Gd}^{3+}$ Chelates. JBIC. 2005, 10, 78-86.

(28) Gregorio, E. Di; Lattuada, L.; Maiocchi, A.; Aime, S.; Ferrauto, G.; Gianolio, E. Supramolecular Adducts between Macrocyclic Gd(III) Complexes and Polyaromatic Systems: A Route to Enhance the Relaxivity through the Formation of Hydrophobic Interactions. Chemical Science 2021, 12, 1368-1377.

(29) Assfalg, M.; Gianolio, E.; Zanzoni, S.; Tomaselli, S.; Russo, V. Lo; Cabella, C.; Ragona, L.; Aime, S.; Molinari, H. Articles NMR Structural Studies of the Supramolecular Adducts between a Liver Cytosolic Bile Acid Binding Protein and Gadolinium(III)-Chelates Bearing Bile Acids Residues: Molecular Determinants of the Binding of a Hepatospecific Magnetic Resonance Imaging Contrast Agent. Journal of Medicinal Chemistry 2007, 50, 5257-5268.

(30) Kannan, R. M.; Nance, E.; Kannan, S.; Tomalia, D. A.; Kannan, A. R. Emerging Concepts in Dendrimer-Based Nanomedicine: From Design Principles to Clinical Applications. Journal of Internal Medicine 2014, 276, 579-617.

(31) Snoussi, K.; Bulte, J. W. M.; Guéron, M.; Van Zijl, P. C. M. Sensitive CEST Agents Based on Nucleic Acid Imino Proton Exchange: Detection of Poly(rU) and of a Dendrimer-Poly(rU) Model for Nucleic Acid Delivery and Pharmacology. Magnetic Resonance in Medicine 2003, 49, 998-1005.

(32) Ali, M. M.; Liu, G.; Shah, T.; Flask, C. A.; And, $\perp$; Pagel, M. D. Using Two Chemical Exchange Saturation Transfer Magnetic Resonance Imaging Contrast Agents for Molecular Imaging Studies. Acc. Chem. Res. 2009, 42 (7), 915-924.

(33) Evbuomwan, O. M.; Kiefer, G.; Sherry, A. D. Amphiphilic EuDOTA-Tetraamide Complexes Form Micelles with Enhanced CEST Sensitivity. European Journal of Inorganic Chemistry 2012, 2126-2134.

(34) Ottiger, M.; Bax, A. Characterization of Magnetically Oriented Phospholipid Micelles for Measurement of Dipolar Couplings in Macromolecules. Journal of Biomolecular NMR 1998, 12, 361-372.

(35) Debroye, E.; Eliseeva, S. V.; Laurent, S.; Vander Elst, L.; Muller, R. N.; Parac-Vogt, 
T. N. Micellar Self-Assemblies of Gadolinium(Iii)/Europium(Iii) Amphiphilic Complexes as Model Contrast Agents for Bimodal Imaging. Dalt. Trans. 2014, 43 (9), 3589-3600.

(36) Terreno, E.; Cabella, C.; Carrera, C.; Delli Castelli, D.; Mazzon, R.; Rollet, S.; Stancanello, J.; Visigalli, M.; Aime, S. From Spherical to Osmotically Shrunken Paramagnetic Liposomes: An Improved Generation of LIPOCEST MRI Agents with Highly Shifted Water Protons. Angew. Chemie - Int. Ed. 2007, 46 (6), 966968.

(37) Opina, A.; Ghaghada, K. B.; Zhao, P.; Kiefer, G.; Annapragada, A. TmDOTATetraglycinate Encapsulated Liposomes as pH-Sensitive LipoCEST Agents. PLoS One 2011, 6 (11), 27370.

(38) Delli Castelli, D.; Terreno, E.; Carrera, C.; Giovenzana, G. B.; Mazzon, R.; Rollet, S.; Visigalli, M.; Aime, S.; Avogadro, A. Lanthanide-Loaded Paramagnetic Liposomes as Switchable Magnetically Oriented Nanovesicles. Concepts Magn. Reson. 2007, 46 (5), 255-265.

(39) Langereis, S.; Keupp, J.; J van Velthoven, J. L.; C de Roos, I. H.; Burdinski, D.; Pikkemaat, J. A.; Grüll, H. A Temperature-Sensitive Liposomal 1 H CEST and 19 F Contrast Agent for MR Image-Guided Drug Delivery. Journal of the American Chemical Society 2009, 131, 1380-1381.

(40) Flament, J.; Geffroy, F.; Medina, C.; Robic, C.; Mayer, J.-F.; Valette, J.; Robert, P.; Port, M.; Le Bihan, D.; Lethimonnier, F.; Boumezbeur, F. In Vivo CEST MR Imaging of U87 Mice Brain Tumor Angiogenesis Using Targeted LipoCEST Contrast Agent at 7 T. Magnetic Resonance in Medicine 2013, 69, 179-187

(41) Burdinski, D.; Pikkemaat, J. A.; Emrullahoglu, M.; Costantini, F.; Verboom, W.; Langereis, S.; Grüll, H.; Huskens, J. Imaging Agents Targeted LipoCEST Contrast Agents for Magnetic Resonance Imaging: Alignment of Aspherical Liposomes on a Capillary Surface. Angewandte Chemie 2013, 49 (12), 2227-2229.

(42) Zhao, J. M.; Har-el, Y.; McMahon, M. T.; Zhou, J.; Dean Sherry, A.; Sgouros, G.; M Bulte, J. W.; M van Zijl, P. C.; Morgan, R. H. Size-Induced Enhancement of Chemical Exchange Saturation Transfer (CEST) Contrast in Liposomes. Journal of the American Chemical Society 2008, 130, 5178-5184.

(43) Ferrauto, G.; Gregorio, E. Di.; Baroni, S.; Aime, S. Frequency-Encoded MRICEST Agents Based on ParamagneticLiposomes/RBC Aggregates. Nano Letters 2014, 14, 6857-6862.

(44) Tripepi, M.; Ferrauto, G.; Aolo Oronzobennardi, P.; Aime, S.; Castelli, D. D. Imaging Agents Multilamellar LipoCEST Agents Obtained from Osmotic Shrinkage of Paramagnetically Loaded Giant Unilamellar Vescicles (GUVs). Angewandte Chemie. 2020, 59, 2279-2283. 
(45) Carniato, F.; Ferrauto, G.; Muñoz-Úbeda, M.; Tei, L. Magnetochemistry Water Diffusion Modulates the CEST Effect on Tb(III)-Mesoporous Silica Probes. Magnetochemistry 2020, 6, 38 .

(46) Mao, X.; Xu, J.; Cui, H. Functional Nanoparticles for Magnetic Resonance Imaging. Wiley Interdiscip. Rev. Nanomedicine Nanobiotechnology 2016, 8 (6), 814-841.

(47) Mao, X.; Xu, J.; Cui, H. Advanced Review Functional Nanoparticles for Magnetic Resonance Imaging. WIREs Nanomed Nanobiotechnol 2016, 8, 814-841.

(48) Bozzuto, G.; Molinari, A. Liposomes as Nanomedical Devices. Int. J. Nanomedicine 2015, 10, 975-999.

(49) Yu, Y.; Liu, X. Silicon-Based Nanoparticles for Drug Delivery. Nanobiomaterials 2017, 379-402.

(50) Haidary, S. M.; Córcoles, E. P.; Ali, N. K. Folic Acid Delivery Device Based on Porous Silicon Nanoparticles Synthesized by Electrochemical Etching. Int. J. Electrochem. Sci 2013, 8, 9956-9966.

(51) Rosenholm, J. M.; Sahlgren, C.; Lind, M. Towards Multifunctional, Targeted Drug Delivery Systems Using Mesoporous Silica Nanoparticles-Opportunities \& Challenges. Nanoscale 2010, 2, 1870-1883.

(52) Slowing II; Vivero-Escoto, J. L.; Wu, C. W.; Lin, V. S. Y. Mesoporous Silica Nanoparticles as Controlled Release Drug Delivery and Gene Transfection Carriers. Adv. Drug Deliv. Rev. 2008, 60 (11), 1278-1288.

(53) Cha, B. G.; Kim, J. Functional Mesoporous Silica Nanoparticles for Bio-Imaging Applications. WIREs Nanomedicine and Nanobiotechnology 2019, 11, 1515.

(54) Stöber, W.; Fink, A.; Bohn, E. Controlled Growth of Monodisperse Silica Spheres in the Micron Size Range. J. Colloid Interface Sci. 1968, 26 (1), 62-69.

(55) Plumeré, N.; Ruff, A.; Speiser, B.; Feldmann, V.; Mayer, H. A. Stöber Silica Particles as Basis for Redox Modifications: Particle Shape, Size, Polydispersity, and Porosity. J. Colloid Interface Sci. 2012, 368 (1), 208-219.

(56) Kim, S.-D.; Surabhi, S.; Choi, J.; Jeong, J.-R. Preparation of Monodisperse Silica Nanoparticles via Controlling the Interphase of Two-Phase Synthesis for Optical Anticounterfeiting Materials. Electron. Mater. Lett. 2019, 15, 3.

(57) Tabisz, Ł.; Stanek, J.; Łęska, B. "DIY" Silica Nanoparticles: Exploring the Scope of a Simplified Synthetic Procedure and Absorbance-Based Diameter Measurements. Materials (Basel). 2020, 13 (14).

(58) Khaleghi, A.; Sadrameli, S. M.; Manteghian, M. Thermodynamic and Kinetics Investigation of Homogeneous and Heterogeneous Nucleation. Rev. Inorg. Chem. 
2020, 40 (4), 167-192.

(59) Hartmann, K. I.; Nieto, A.; Wu, E. C.; Freeman, W. R.; Kim, J. S.; Chhablani, J.; Sailor, M. J.; Cheng, L. Hydrosilylated Porous Silicon Particles Function as an Intravitreal Drug Delivery System for Daunorubicin. J. Ocul. Pharmacol. Ther. 2013, 29 (5), 493-500.

(60) Lou, X. W.; Archer, L. A.; Yang, Z. Hollow Micro-/Nanostructures: Synthesis and Applications. Advanced Materials. 2008, 20, 3987-4019.

(61) Zhang, Q.; Zhang, T.; Ge, J.; Yin, Y. Permeable Silica Shell through SurfaceProtected Etching. Nano Letters 2008, 8, 2867-2871.

(62) Chen, Y.; Chen, H.; Zeng, D.; Tian, Y.; Chen, F.; Feng, J.; Shi, J. Core / Shell Structured Hollow Mesoporous Nanocapsules: A Potential Platform for Simultaneous Cell Imaging and Anticancer Drug Delivery. ACS Nano. 2010, 4 (10), 6001-6013.

(63) Bazuła, P. A.; Lu, A. H.; Nitz, J. J.; Schüth, F. Surface and Pore Structure Modification of Ordered Mesoporous Carbons via a Chemical Oxidation Approach. Microporous Mesoporous Mater. 2008, 108 (1-3), 266-275.

(64) Gu“ttel, R. G.; Paul, M.; Schu“th, F. S. Ex-Post Size Control of High-TemperatureStable Yolk-Shell Au, @ZrO 2. Journal of Catalysis 2012, 289, 100-104.

(65) A.J. Lecloux, J. Bronckart, F. Noville, C. Dodet, P. Marchot, J. P. P. Study of the Texture of Monodisperse Silica Sphere Samples in the Nanometer Size Range. Colloids and Surfaces 1986, 19 (2-3), 359-374.

(66) Vrij, A. Introduction, I. Synthesis and Characterization of Monodisperse Spheres Colloidal. J. Colloid Interface Sci. 1993, 156, 1-18.

(67) Green, D. L.; Lin, J. S.; Lam, Y. F.; Hu, M. Z. C.; Schaefer, D. W.; Harris, M. T. Size, Volume Fraction, and Nucleation of Stober Silica Nanoparticles. J. Colloid Interface Sci. 2003, 266 (2), 346-358.

(68) Drummond, C.; McCann, R.; Patwardhan, S. V. A Feasibility Study of the Biologically Inspired Green Manufacturing of Precipitated Silica. Chem. Eng. J. 2014, 244, 483-492.

(69) Guerrero-Martínez, A.; Pérez-Juste, J.; Liz-Marzán, L. M. Recent Progress on Silica Coating of Nanoparticles and Related Nanomaterials. Advanced Materials 2010, 22, 1182-1195.

(70) Ren, G.; Su, H.; Wang, S. The Combined Method to Synthesis Silica Nanoparticle by Stöber Process. J. Sol-Gel Sci. Technol. 2020, 96 (1), 108-120.

(71) Zhao, P.; Yang, D.; Chmelka, P.; Stucky, B. F. Morphological Control of Highly 
Ordered Mesoporous Silica SBA-15. J. Am. Chem. Soc 1992, 114 (1), 275-279.

(72) Wang, J.; Xiao, Q.; Zhou, H.; Sun, P.; Yuan, Z.; Li, B.; Ding, D.; Shi, A. C.; Chen, T. Budded, Mesoporous Silica Hollow Spheres: Hierarchical Structure Controlled by Kinetic Self-Assembly. Adv. Mater. 2006, 18 (24), 3284-3288.

(73) Alfredsson, V.; Keung, M.; Monnier, A.; Stucky, G. D.; Unger, K. K.; Schüth, F. High-Resolution Transmission Electron Microscopy of Mesoporous MCM-41 Type Materials. J. Chem. Soc. Chem. Commun. 1994, 8, 921-922.

(74) Johansson, E. M.; Cordoba, J.M.; Oden, M. Controlling the Pore Size and Morphology of Mesoporous Silica. Materials Letters 2009, 63, 2129-2131.

(75) Alonso, B.; Clinard, C.; Durand, D.; Véron, E.; Massiot, D. New Routes to Mesoporous Silica-Based Spheres with Functionalised Surfaces. Chemical Communications 2005, 1746-1748.

(76) Lin, Y. S.; Haynes, C. L. Impacts of Mesoporous Silica Nanoparticle Size, Pore Ordering, and Pore Integrity on Hemolytic Activity. J. Am. Chem. Soc. 2010, 132 (13), 4834-4842.

(77) Wu, S.-H.; Mou, C.-Y.; Lin, H.-P. Synthesis of Mesoporous Silica Nanoparticles. Chem. Soc. Rev. 2013, 42 (9), 3862.

(78) Zhang, Q.; Ge, J.; Goebl, J.; Hu, Y.; Lu, Z.; Yin, Y. Rattle-Type Silica Colloidal Particles Prepared by a Surface-Protected Etching Process. Nano Res. 2009, 2 (7), 583-591.

(79) Narayan, R.; Nayak, U. Y.; Raichur, A. M.; Garg, S. Pharmaceutics Mesoporous Silica Nanoparticles: A Comprehensive Review on Synthesis and Recent Advances. Pharmaceutics 2018, 10, 118.

(80) Yuan, D.; Ellis, C. M.; Davis, J. J. Materials Mesoporous Silica Nanoparticles in Bioimaging. Materials 2020, 13, 3795.

(81) D'Arrigo, JS. Screening of membrane surface charges by divalent cations: an atomic representation. Am J Physiol. 1978, 235 (3), 109-117.

(82) Croissant, J. G.; Fatieiev, Y.; Khashab, N. M. Degradability and Clearance of Silicon, Organosilica, Silsesquioxane, Silica Mixed Oxide, and Mesoporous Silica Nanoparticles. Adv. Mater. 2017, 29 (9).

(83) Pal, N.; Lee, J.-H.; Cho, E.-B. Recent Trends in Morphology-Controlled Synthesis and Application of Mesoporous Silica Nanoparticles. Nanomaterials 2020, 10, 2122.

(84) Yamada, H.; Urata, C.; Aoyama, Y.; Osada, S.; Yamauchi, Y.; Kuroda, K. Preparation of Colloidal Mesoporous Silica Nanoparticles with Different Diameters 
and Their Unique Degradation Behavior in Static Aqueous Systems. Chem. Mater. 2012, 24 (8), 1462-1471.

(85) Vartuli, J. C.; Schmitt, K. D.; Kresge, C. T.; Roth, W. J.; Leonowicz, M. E.; McCullen, S. B.; Hellring, S. D.; Beck, J. S.; Schlenker, J. L.; Olson, D. H.; Sheppard, E. W. Effect of Surfactant/Silica Molar Ratios on the Formation of Mesoporous Molecular Sieves: Inorganic Mimicry of Surfactant Liquid-Crystal Phases and Mechanistic Implications. Chem. Mater. 1994, 6 (12), 2317-2326.

(86) Ozin, G. A. Morphogenesis of Biomineral and Morphosynthesis of Biomimetic Forms. Acc. Chem. Res. 1997, 30 (1), 17-27.

(87) Ishii, Y.; Nishiwaki, Y.; Al-Zubaidi, A.; Kawasaki, S. Pore Size Determination in Ordered Mesoporous Materials Using Powder X-Ray Diffraction. J. Phys. Chem. C 2013, 117 (35), 18120-18130.

(88) Qiao, Z.-A.; Zhang, L.; Guo, M.; Liu, Y.; Huo, Q. Synthesis of Mesoporous Silica Nanoparticles via Controlled Hydrolysis and Condensation of Silicon Alkoxide. Chem. Mater 2009, 21, 3823.

(89) Altın, B.; Gradzielski, M. Synthesis and Characterization of Silica Based Functional Nanoparticles for Multi-Purpose Applications. Master of Science, Izmir Institute of Technology 2009.

(90) Kobler, J.; Möller, K.; Bein, T. Colloidal Suspensions of Functionalized Mesoporous Silica Nanoparticles. ACS Nano 2008, 2 (4), 791-799.

(91) Soten, I.; Ozin, G. Supramolecular Organization and Materials Design. Cambridge University Press, 2009.

(92) Raman, N. K.; Anderson, M. T.; Brinker, C. J. Template-Based Approaches to the Preparation of Amorphous, Nanoporous Silicas. Chem. Mater. 1996, 8 (8), 16821701

(93) Bhattacharyya, S.; Lelong, G.; Saboungi, M. L. Recent Progress in the Synthesis and Selected Applications of MCM-41: A Short Review. J. Exp. Nanosci. 2006, 1 (3), 375-395.

(94) Dawib, Y. A. NMR T 1 Studies of Colloidal Unimolecular Polymer ( CUP ) Surface Water. Ph.D. Thesis, Missouri University of Science and Technology 2015.

(95) Möller, K.; Bein, T. Talented Mesoporous Silica Nanoparticles. Chem. Mater. 2017, 29 (1), 371-388.

(96) Wang, J.; Sugawara-Narutaki, A.; Fukao, M.; Yokoi, T.; Shimojima, A.; Okubo, T. Two-Phase Synthesis of Monodisperse Silica Nanospheres with Amines or Ammonia Catalyst and Their Controlled Self-Assembly. ACS Appl. Mater. Interfaces 2011, 3 (5), 1538-1544. 
(97) Zhou, X.; Cheng, X.; Feng, W.; Qiu, K.; Chen, L.; Nie, W.; Yin, Z.; Mo, X.; Wang, H.; He, C. Synthesis of Hollow Mesoporous Silica Nanoparticles with Tunable Shell Thickness and Pore Size Using Amphiphilic Block Copolymers as Core Templates. Dalt. Trans. 2014, 43 (31), 11834-11842.

(98) Zargar, M.; Hartanto, Y.; Jin, B.; Dai, S. Hollow Mesoporous Silica Nanoparticles: A Peculiar Structure for Thin Film Nanocomposite Membranes. J. Memb. Sci. 2016, $519,1-10$.

(99) Li, T.; Geng, T.; Md, A.; Banerjee, P.; Wang, B. Novel Scheme for Rapid Synthesis of Hollow Mesoporous Silica Nanoparticles (HMSNs) and Their Application as an Efficient Delivery Carrier for Oral Bioavailability Improvement of Poorly WaterSoluble BCS Type II Drugs. Colloids Surfaces B Biointerfaces 2019, 176, 185-193.

(100) Rahman, I. A.; Padavettan, V. Synthesis of Silica Nanoparticles by Sol-Gel: SizeDependent Properties, Surface Modification, and Applications in Silica-Polymer Nanocompositesa Review. Journal of Nanomaterials 2012, 15.

(101) Singh, L. P.; Bhattacharyya, S. K.; Mishra, G.; Ahalawat, S. Functional Role of Cationic Surfactant to Control the Nano Size of Silica Powder. Applied Nanoscience 2011, 1, 117-122.

(102) Fuertes, A. B.; Valle-Vigón, P.; Sevilla, M. Synthesis of Colloidal Silica Nanoparticles of a Tunable Mesopore Size and Their Application to the Adsorption of Biomolecules. J. Colloid Interface Sci. 2010, 349 (1), 173-180.

(103) Kim, M. H.; Na, H. K.; Kim, Y. K.; Ryoo, S. R.; Cho, H. S.; Lee, K. E.; Jeon, H.; Ryoo, R.; Min, D. H. Facile Synthesis of Monodispersed Mesoporous Silica Nanoparticles with Ultralarge Pores and Their Application in Gene Delivery. ACS Nano. 2011, 5 (5), 3568-3576.

(104) Hah, H. J.; Kim, J. S.; Jeon, B. J.; Koo, S. M.; Lee, Y. E. Simple Preparation of Monodisperse Hollow Silica Particles without Using Templates. Chem. Commun. 2003, 3 (14), 1712-1713.

(105) Fuji, M.; Han, Y. S.; Takai, C. Synthesis and Applications of Hollow Particles. KONA Powder Part. J. 2012, 30 (30), 47-68.

(106) Bao, Y.; Shi, C.; Wang, T.; Li, X.; Ma, J. Recent Progress in Hollow Silica: Template Synthesis, Morphologies and Applications. Microporous and Mesoporous Materials 2016, 1, 121-136.

(107) Kim, K. Do.; Choi, K. Y.; Yang, J. W. Formation of Spherical Hollow Silica Particles from Sodium Silicate Solution by Ultrasonic Spray Pyrolysis Method. Colloids Surfaces A Physicochem. Eng. Asp. 2005, 254 (1-3), 193-198.

(108) Chung, Y. S.; Lim, J. S.; Park, S. B.; Okuyama, K. Templated Synthesis of Silica 
Hollow Particles by Using Spray Pyrolysis. J. Chem. Eng. Japan 2004, 37 (9), 10991104.

(109) Nandiyanto, A. B. D.; Okuyama, K. Progress in Developing Spray-Drying Methods for the Production of Controlled Morphology Particles: From the Nanometer to Submicrometer Size Ranges. Adv. Powder Technol. 2011, 22 (1), 1-19.

(110) Cheow, W. S.; Li, S.; Hadinoto, K. Spray Drying Formulation of Hollow Spherical Aggregates of Silica Nanoparticles by Experimental Design. Chem. Eng. Res. Des. 2010, 88 (5-6), 673-685.

(111) Sharma, J.; Polizos, G. Hollow Silica Particles: Recent Progress and Future Perspectives. Nanomaterials 2020, 1, 1-22.

(112) Wang, J.; Pan, M.; Yuan, J.; Lin, Q.; Zhang, X.; Liu, G.; Zhu, L. Hollow Mesoporous Silica with a Hierarchical Shell from: In Situ Synergistic Soft-Hard Double Templates. Nanoscale 2020, 12 (19), 10863-10871.

(113) Song, L.; Ge, X.; Zhang, Z. Interfacial Fabrication of Silica Hollow Particles in a Reverse Emulsion System. Chem. Lett. 2005, 34 (10), 1314-1315.

(114) Chen, J.; Wu, X.; Hou, X.; Su, X.; Chu, Q.; Fahruddin, N.; Zhao, J. X. ShapeTunable Hollow Silica Nanomaterials Based on a Soft-Templating Method and Their Application as a Drug Carrier. ACS Appl. Mater. Interfaces 2014, 6 (24), 21921-21930.

(115) Akhtar, S.; Günday, Ş. T.; Jermy, B. R.; Almessiere, M. A.; Bozkurt, A. A Novel Approach to Produce Monodisperse Hollow Pure Silica Spheres. J. Saudi Chem. Soc. 2019, 23 (4), 477-485.

(116) Nomura, T.; Morimoto, Y.; Ishikawa, M.; Tokumoto, H.; Konishi, Y. Synthesis of Hollow Silica Microparticles from Bacterial Templates. Adv. Powder Technol. 2010, 21 (2), 218-222.

(117) Liao, S.; Lin, L.; Chen, X.; Liu, J.; Zhang, B. Fabrication of Silica Hollow Particles Using Yeast Cells as a Template. AIP Conf. Proc. 2018, 1955, 30027.

(118) Yue, Q.; Sun, J.; Kang, Y. Advances in the Interfacial Assembly of Mesoporous Silica on Magnetite Particles. Angewandte Chemie 2020, 15936-15949.

(119) Binks, B. P. Relationship between Microemulsion Phase Behavior and Macroemulsion Type in Systems Containing Nonionic Surfactant. Langmuir 1993, 9 (1), 25-28.

(120) Ikari, K.; Suzuki, K.; Imai, H. Structural Control of Mesoporous Silica Nanoparticles in a Binary Surfactant System. Langmuir 2006, 22 (2), 802-806.

(121) Li, M.; Zhang, C.; Yang, X. L.; Xu, H. B. Controllable Synthesis of Hollow 
Mesoporous Silica Nanoparticles Templated by Kinetic Self-Assembly Using a Gemini Surfactant. RSC Adv. 2013, 3 (37), 16304-16307.

(122) Winsor, P. A. Hydrotropy, Solubilisation and Related Emulsification Processes. Part I. Trans. Faraday Soc. 1948, 44, 376-398.

(123) Zhang, H.; Xu, H.; Wu, M.; Zhong, Y.; Wang, D.; Jiao, Z. A Soft-Hard Template Approach towards Hollow Mesoporous Silica Nanoparticles with Rough Surfaces for Controlled Drug Delivery and Protein Adsorption. J. Mater. Chem. B 2015, 3 (31), 6480-6489.

(124) Chen, Y.; Chen, H.; Guo, L.; He, Q.; Chen, F.; Zhou, J.; Feng, J.; Shi, J.

Hollow/Rattle-Type Mesoporous Nanostructures by a Structural Difference-Based Selective Etching Strategy. ACS 2010, 4 (1), 529-539.

(125) Li, Y.; Li, N.; Pan, W.; Yu, Z.; Yang, L.; Tang, B. Hollow Mesoporous Silica Nanoparticles with Tunable Structures for Controlled Drug Delivery. ACS Appl. Mater. Interfaces 2017, 9 (3), 2123-2129.

(126) Bharti, C.; Gulati, N.; Nagaich, U.; Pal, A. Mesoporous Silica Nanoparticles in Target Drug Delivery System: A Review. Int. J. Pharm. Investig. 2015, 5 (3), 124.

(127) Park, I.; Ko, S. H.; An, Y. S.; Choi, K. H.; Chun, H.; Lee, S.; Kim, G. Monodisperse Polystyrene-Silica Core-Shell Particles and Silica Hollow Spheres Prepared by the Stöber Method. Journal of Nanoscience and Nanotechnology 2009, 9, 7224-7228.

(128) Bao, Y.; Wang, T.; Kang, Q.; Shi, C.; Ma, J. Micelle-Template Synthesis of Hollow Silica Spheres for Improving Water Vapor Permeability of Waterborne Polyurethane Membrane. Nat. Publ. Gr. 2017, 7, 46638.

(129) Hadipour Moghaddam, S. P.; Yazdimamaghani, M.; Ghandehari, H. GlutathioneSensitive Hollow Mesoporous Silica Nanoparticles for Controlled Drug Delivery. $J$. Control. Release 2018, 282, 62-75.

(130) Brunauer, S.; Emmett, P. H.; Teller, E. Adsorption of Gases in Multimolecular Layers. J. Am. Chem. Soc. 1938, 60 (2), 309-319.

(131) Barrett, E. P.; Joyner, L. G.; Halenda, P. P. The Determination of Pore Volume and Area Distributions in Porous Substances. I. Computations from Nitrogen Isotherms. J. Am. Chem. Soc. 1951, 73 (1), 373-380.

(132) Nozawa, K.; Gailhanou, H.; Raison, L.; Panizza, P.; Ushiki, H.; Sellier, E.; Delville, J. P.; Delville, M. H. Smart Control of Monodisperse Stöber Silica Particles: Effect of Reactant Addition Rate on Growth Process. Langmuir 2005, 21 (4), 1516-1523.

(133) Jafarzadeh, M.; Rahman, A. I. A.; Sipaut, A. C. S. Synthesis of Silica Nanoparticles by Modified Sol-Gel Process: The Effect of Mixing Modes of the Reactants and Drying Techniques. Journal of Sol-Gel Science and Technology 2009, 50, 328-336. 
(134) Gregory, J. K.; Clary, D. C.; Liu, K.; Brown, M. G.; Saykally, R. J. The Water Dipole Moment in Water Clusters. Science 1997, 275 (5301), 814-817.

(135) Khan, I.; Saeed, K.; Khan, I. Nanoparticles: Properties, Applications and Toxicities. Arab. J. Chem. 2019, 12, 908-931.

(136) Fernandes, R. S.; Raimundo, I. M.; Pimentel, M. F. Revising the Synthesis of Stöber Silica Nanoparticles: A Multivariate Assessment Study on the Effects of Reaction Parameters on the Particle Size. Colloids Surfaces A Physicochem. Eng. Asp. 2019, 577, $1-7$.

(137) Clarke, S. Development of Hierarchical Magnetic Nanocomposite Materials for Biomedical Applications. Ph.D. Thesis, Dublin City University 2013.

(138) Greasley, S. L.; Page, S. J.; Sirovica, S.; Chen, S.; Martin, R. A.; Riveiro, A.; Hanna, J. V.; Porter, A. E.; Jones, J. R. Controlling Particle Size in the Stöber Process and Incorporation of Calcium. J. Colloid Interface Sci. 2016, 469, 213-223.

(139) Le, Y.; Chen, J. F.; Wang, J. X.; Shao, L.; Wang, W. C. A Novel Pathway for Synthesis of Silica Hollow Spheres with Mesostructured Walls. Mater. Lett. 2004, 58 (15), 2105-2108.

(140) Feng, J.; Wang, Z.; Shen, B.; Zhang, L.; Yang, X.; He, N. Effects of Template Removal on Both Morphology of Mesoporous Silica-Coated Gold Nanorod and Its Biomedical Application. RSC Adv. 2014, 4 (54), 28683-28690.

(141) Rahman, I. A.; Padavettan, V. Synthesis of Silica Nanoparticles by Sol-Gel: SizeDependent Properties, Surface Modification, and Applications in Silica-Polymer Nanocomposites-A Review. J. Nanomater. 2012, 1-15.

(142) Puddu, V. P.; Perry, C. C. Peptide Adsorption on Silica Nanoparticles: Evidence of Hydrophobic Interactions. ACS Nano 2012, 6 (7), 6356-6363.

(143) She, X.; Chen, L.; Li, C.; He, C.; He, L.; Kong, L. Functionalization of Hollow Mesoporous Silica Nanoparticles for Improved 5-Fu Loading. J. Nanomater. 2015, $1-9$.

(144) Doadrio, J. C.; Sousa, E. M. B.; Izquierdo-Barba, I.; Doadrio, A. L.; Perez-Pariente, J.; Vallet-Regí, M. Functionalization of Mesoporous Materials with Long Alkyl Chains as a Strategy for Controlling Drug Delivery Pattern. J. Mater. Chem. 2006, $462-466$.

(145) Huh, S.; Wiench, J. W.; Trewyn, B. G.; Song, S.; Pruski, M.; Lin, V. S.-Y. Tuning of Particle Morphology and Pore Properties in Mesoporous Silicas with Multiple Organic Functional Groups. Chem. Commun. 2003, 3 (18), 2364-2365.

(146) Lim, M. H.; Stein, A. Comparative Studies of Grafting and Direct Syntheses of Inorganic-Organic Hybrid Mesoporous Materials. Chem. Mater. 1999, 11, 3285- 
3295.

(147) Rose, P. A.; Praseetha, P. K.; Bhagat, M.; Alexander, P.; Abdeen, S.; Chavali, M. Drug Embedded PVP Coated Magnetic Nanoparticles for Targeted Killing of Breast Cancer Cells. Technol. Cancer Res. Treat. 2013, 12 (5), 463-472.

(148) Rodriguez-Ruiz, V.; Maksimenko, A.; Anand, R.; Monti, S.; Agostoni, V.; Couvreur, P.; Lampropoulou, M.; Yannakopoulou, K.; Gref, R. Efficient "Green" Encapsulation of a Highly Hydrophilic Anticancer Drug in Metal-Organic Framework Nanoparticles. J Drug Target 2015, 23 (7-8), 759-767.

(149) Pinelli, F.; Perale, G.; Rossi, F. Coating and Functionalization Strategies for Nanogels and Nanoparticles for Selective Drug Delivery. Gels 2020, 6 (1).

(150) Mine, E.; Yamada, A.; Kobayashi, Y.; Konno, M.; Liz-Marzán, L. M. Direct Coating of Gold Nanoparticles with Silica by a Seeded Polymerization Technique. J. Colloid Interface Sci. 2003, 264 (2), 385-390.

(151) Liu, S.; Han, M.-Y. Silica-Coated Metal Nanoparticles. Chem. - An Asian J. 2010, 5 (1), 36-45.

(152) Graf, C.; Vossen, D. L. J.; Imhof, A.; Van Blaaderen, A. A General Method to Coat Colloidal Particles with Silica. Langmuir 2003, 19 (17), 6693-6700.

(153) Hristov, D. R.; Rocks, L.; Kelly, P. M.; Thomas, S. S.; Pitek, A. S.; Verderio, P.; Mahon, E.; Dawson, K. A. Tuning of Nanoparticle Biological Functionality through Controlled Surface Chemistry and Characterisation at the Bioconjugated Nanoparticle Surface. Sci. Rep. 2015, 5, 17040.

(154) Zhuravlev, L. T. The Surface Chemistry of Amorphous Silica. Zhuravlev Model. Colloids Surfaces A Physicochem. Eng. Asp. 2000, 173 (1-3), 1-38.

(155) Iler, R. K. The Chemistry of Silica: Solubility, Polymerization, Colloid and Surface Properties, and Biochemistry. Colloid Surf. 1979, 172-311.

(156) Björklund, S.; Kocherbitov, V. Alcohols React with MCM-41 at Room Temperature and Chemically Modify Mesoporous Silica. Scientific Reports 2017, 7.

(157) Jeelani, P. G.; Mulay, P.; Venkat, R.; Ramalingam, C. Multifaceted Application of Silica Nanoparticles. A Review. Silicon 2020, 12 (6), 1337-1354.

(158) Hwang, N.-M.; Jung, J.-S.; Lee, D.-K. Thermodynamics and Kinetics in the Synthesis of Monodisperse Nanoparticles. Thermodynamics - Fundamentals and Its Application in Science $\mathbf{2 0 1 2 .}$

(159) Klabunde, K. J.; Erickson, L.; Koper, O.; Richards, R. Review of Nanoscale Materials in Chemistry: Environmental Applications. ACS 2010, 1045.

(160) Han, Y.; Lu, Z.; Teng, Z.; Liang, J.; Guo, Z.; Wang, D.; Han, M.-Y.; Yang, W. 
Unraveling the Growth Mechanism of Silica Particles in the Stöber Method: In Situ Seeded Growth Model. Langmuir 2017, 33 (23), 5879-5890.

(161) Brinker, C. J. Hydrolysis and Condensation of Silicates: Effects on Structure. J. Non. Cryst. Solids 1988, 100 (3), 31-50.

(162) Zha, J.; Roggendorf, H. Sol-Gel Science, the Physics and Chemistry of Sol-Gel Processing, Adv. Mater. 1991, 3 (10), 522-522.

(163) Semantic Scholar, accessed Jul 12 2021, <https://www.semanticscholar.org/paper/3.2.1.-Fundamentals-of-HomogeneousNucleation>.

(164) Sang, M. C.; Lee, M.; Kim, W. S. Preparation of Large Monodispersed Spherical Silica Particles Using Seed Particle Growth. J. Colloid Interface Sci. 2005, 286 (2), 536-542.

(165) Wang, L.; Sasaki, T.; Ebina, Y.; Kurashima, K.; Watanabe, M. Fabrication of Controllable Ultrathin Hollow Shells by Layer-by-Layer Assembly of Exfoliated Titania Nanosheets on Polymer Templates. Chem. Mater. 2002, 14 (11), 4827-4832.

(166) Quan, B.; Lee, C.; Yoo, J. S.; Piao, Y. Facile Scalable Synthesis of Highly Monodisperse Small Silica Nanoparticles Using Alkaline Buffer Solution and Their Application for Efficient Sentinel Lymph Node Mapping. J. Mater. Chem. 2017, 5 (3), 586-594.

(167) Colard, C. A. L.; Cave, R. A.; Grossiord, N.; Covington, J. A.; Bon, S. A. F. Conducting Nanocomposite Polymer Foams from Lce-Crystal-Templated Assembly of Mixtures of Colloids. Adv. Mater. 2009, 21 (28), 2894-2898.

(168) Lin, Y. H.; Su, K. L.; Tsai, P. S.; Chuang, F. L.; Yang, Y. M. Fabrication and Characterization of Transparent Superhydrophilic/Superhydrophobic Silica Nanoparticulate Thin Films. Thin Solid Films 2011, 519 (16), 5450-5455.

(169) Dabbaghian, M. A.; Babalou, A. A.; Hadi, P.; Jannatdoust, E. A Parametric Study of the Synthesis of Silica Nanoparticles via Sol-Gel Precipitation Method. Int. J. Nanosci. Nanotechnol. 2010, 6 (2), 104-113.

(170) Liu, D.; Bimbo, L. M.; Mäkilä, E.; Villanova, F.; Kaasalainen, M.; Herranz-Blanco, B.; Caramella, C. M.; Lehto, V. P.; Salonen, J.; Herzig, K. H.; Hirvonen, J.; Santos, H. A. Co-Delivery of a Hydrophobic Small Molecule and a Hydrophilic Peptide by Porous Silicon Nanoparticles. J. Control. Release 2013, 170 (2).

(171) Tang, F.; Li, L.; Chen, D. Mesoporous Silica Nanoparticles: Synthesis, Biocompatibility and Drug Delivery. Adv. Mater. 2012, 24 (12), 1504-1534.

(172) Huang, X.; Peng, Z.; Zhong, Y.; Li, Q.; Li, X.; N-t, N.; Zhao, T.; Nguyen, N.-T.; Xie, Y.; Sun, X. Inorganic Nanocrystals Functionalized Mesoporous Silica 
Nanoparticles: Fabrication and Enhanced Bio-Applications. Front. Chem 2017, 5, 118.

(173) Tan, L.; Chen, D.; Liu, H.; Tang, F. A Silica Nanorattle with a Mesoporous Shell: An Ideal Nanoreactor for the Preparation of Tunable Gold Cores. Adv. Mater. 2010, 22 (43), 4885-4889.

(174) Okamoto, M.; Tsukada, H.; Fukasawa, S.; Sakajiri, A. Synthesis of Hollow and Rattle-Type Mesoporous Silica Spheres by Treating Layered Mesoporous Silica with a Basic Solution, and Using the Spheres as Microreactors for Two-Phase Reactions. Journal of Materials Chemistry 2015, 3, 11880-11890.

(175) Priebe, M.; Fromm, K. M. Nanorattles or Yolk-Shell Nanoparticles-What Are They, How Are They Made, and What Are They Good For? Chem. - A Eur. J. 2014, 21 (10), 3854-3874. 